\title{
Spatial Heterogeneity, Source-Sink Dynamics, and the Local Coexistence of Competing Species
}

\author{
Priyanga Amarasekare ${ }^{1, *}$ and Roger M. Nisbet ${ }^{1,2, \uparrow}$ \\ 1. National Center for Ecological Analysis and Synthesis, \\ University of California, Santa Barbara, California 93101-5504; \\ 2. Department of Ecology, Evolution, and Marine Biology, \\ University of California, Santa Barbara, California 93106 \\ Submitted September 18, 2000; Accepted June 19, 2001
}

\begin{abstract}
AвSTRACT: Patch occupancy theory predicts that a trade-off between competition and dispersal should lead to regional coexistence of competing species. Empirical investigations, however, find local coexistence of superior and inferior competitors, an outcome that cannot be explained within the patch occupancy framework because of the decoupling of local and spatial dynamics. We develop two-patch metapopulation models that explicitly consider the interaction between competition and dispersal. We show that a dispersal-competition trade-off can lead to local coexistence provided the inferior competitor is superior at colonizing empty patches as well as immigrating among occupied patches. Immigration from patches that the superior competitor cannot colonize rescues the inferior competitor from extinction in patches that both species colonize. Too much immigration, however, can be detrimental to coexistence. When competitive asymmetry between species is high, local coexistence is possible only if the dispersal rate of the inferior competitor occurs below a critical threshold. If competing species have comparable colonization abilities and the environment is otherwise spatially homogeneous, a superior ability to immigrate among occupied patches cannot prevent exclusion of the inferior competitor. If, however, biotic or abiotic factors create spatial heterogeneity in competitive rankings across the landscape, local coexistence can occur even in the absence of a dispersal-competition trade-off. In fact, coexistence requires that the dispersal rate of the overall inferior competitor not exceed a critical threshold. Explicit consideration of how dispersal modifies local competitive interactions shifts the focus from the patch occupancy approach with its emphasis on extinctioncolonization dynamics to the realm of source-sink dynamics. The key to coexistence in this framework is spatial variance in fitness. Unlike in the patch occupancy framework, high rates of dispersal

\footnotetext{
* Present address: Department of Ecology and Evolution, University of Chicago, Chicago, Illinois 60637-1573; e-mail: amarasek@uchicago.edu.

†E-mail: nisbet@lifesci.ucsb.edu.
}

Am. Nat. 2001. Vol. 158, pp. 572-584. (c) 2001 by The University of Chicago. 0003-0147/2001/15806-0002\$03.00. All rights reserved. can undermine coexistence, and hence diversity, by reducing spatial variance in fitness.

Keywords: competition, coexistence, spatial heterogeneity, source-sink dynamics, immigration, dispersal-competition trade-off.

The issue of how species coexist in patchy environments is central to both basic and applied ecology. When competition for resources is asymmetric, a life-history tradeoff between competitive and dispersal abilities can lead to coexistence in a patchy environment (Skellem 1951). This idea has been formalized in the patch occupancy metapopulation framework (Levins 1969, 1970). The patch occupancy approach assumes that local competitive interactions occur on a much faster time scale relative to extinction-colonization dynamics (Cohen 1970; Levins and Culver 1971; Slatkin 1974; Hastings 1980; Nee and May 1992; Tilman et al. 1994). For instance, when patches are colonized by both superior and inferior competitors, there is rapid exclusion of the inferior species. This restricts the inferior competitor to patches that the superior competitor cannot colonize. The predicted outcome is regional coexistence, with the two species occupying mutually exclusive subsets of patches in the metapopulation.

Empirical studies of dispersal-competition trade-offs, however, reveal a pattern that is at odds with the theoretical prediction of regional coexistence. For instance, in Lei and Hanski's (1998) study of two parasitoid species that attack the butterfly Melitaea cinxia, the superior competitor ( $\mathrm{Co}$ tesia melitaearum) was absent from some host populations, but the superior disperser (Hyposoter horticola) was present in all populations sampled. Another host-parasitoid system consisting of the harlequin bug (Murgantia histrionica) and its two egg parasitoids (Trissolcus murgantiae and Ooencyrtus johnsonii) also shows a similar pattern with local coexistence in some patches and the superior competitor absent in other patches (Amarasekare 2000a, 2000b).

The mismatch between patch occupancy theory and data may arise from the separation of time scales inherent in the patch occupancy framework. The assumption that local dynamics occur on a faster time scale relative to 
spatial dynamics restricts the role of dispersal to colonizing empty, or locally extinct, patches. In the absence of any immigration among occupied patches, dispersal cannot influence local competitive interactions. This decoupling of local and spatial dynamics eliminates any possibility of local coexistence. Empirical observations of local coexistence, however, suggest that dispersal may be sufficiently rapid to counteract competitive exclusion. In fact, the two parasitoid species that attack $M$. cinxia appear to exhibit a dispersal-competition trade-off most convincingly at the scale of larval groups within local populations (Lei and Hanski 1998). Movement between such larval groups is likely to occur on a time scale comparable to local competitive interactions. The harlequin bug and its two parasitoids move among populations on a pergeneration basis (Amarasekare 2000a, 2000b). Harrison et al.'s (1995) study of the insect herbivores of ragwort (Senecio jacobea) also demonstrated rapid dispersal of the cinnabar moth (Tyria jacobeae) and other herbivores (Botanophila seneciella and Contarina jacobeae) relative to the time scale of local competition.

These data present a puzzle for theory. Can a dispersalcompetition trade-off lead to local coexistence when competition and dispersal operate on comparable time scales? Answering this question necessitates a shift in focus from extinction-colonization dynamics to source-sink dynamics. For instance, the issue now is not whether a superior ability to colonize empty patches prevents regional exclusion but whether a superior ability to immigrate among occupied patches prevents local exclusion.

\section{The Model}

We use a two-patch model with Lotka-Volterra competitive dynamics within patches and emigration and immigration between patches. The dynamics are given by the following system of equations:

$$
\begin{gathered}
\frac{d X_{i}}{d t}=r_{x} X_{i}\left(1-\frac{X_{i}}{K_{x, i}}-\phi_{x, i} \frac{Y_{i}}{K_{x, i}}\right)+d_{x}\left(X_{j}-X_{i}\right), \\
\frac{d Y_{i}}{d t}=r_{y} Y_{i}\left(1-\frac{Y_{i}}{K_{y, i}}-\phi_{y, i} \frac{X_{i}}{K_{y, i}}\right)+d_{y}\left(Y_{j}-Y_{i}\right), \\
i, j=1,2, \quad i \neq j,
\end{gathered}
$$

where $X_{i}$ and $Y_{i}$ are the abundances of each species in patch $i ; \phi_{x, i}$ and $\phi_{y, i}$ are the competition coefficients, and $K_{x, i}$ and $K_{y, i}$ are the carrying capacities for species 1 and 2 in patch $i ; r_{x}$ and $r_{y}$ are the per capita growth rates, and $d_{x}$ and $d_{y}$ are the per capita emigration rates of species 1 and 2 , respectively.
We nondimensionalize equation (1) in order to describe the system in terms of a minimal set of parameters (Murray 1993). The following transformations,

$$
\begin{aligned}
& x_{i}=\frac{X_{i}}{K_{x, i}}, \quad y_{i}=\frac{Y_{i}}{K_{y, i}}, \quad \tau=r_{x} t, \\
& a_{x, i}=\phi_{x, i} \frac{K_{y, i}}{K_{x, i}}, \quad a_{y, i}=\phi_{y, i} \frac{K_{x, i}}{K_{y, i}}, \\
& k_{x}=\frac{K_{x, j}}{K_{x, i}}, \quad k_{y}=\frac{K_{y, j}}{K_{y, i}}, \quad \rho=\frac{r_{y}}{r_{x}}, \\
& \beta_{x}=\frac{d_{x}}{r_{x}}, \quad \beta_{y}=\frac{d_{y}}{r_{y}},
\end{aligned}
$$

yield the nondimensional system

$$
\begin{gathered}
\frac{d x_{i}}{d \tau}=x_{i}\left(1-x_{i}-a_{x, i} y_{i}\right)+\beta_{x}\left(k_{x} x_{j}-x_{i}\right), \\
\frac{d y_{i}}{d \tau}=\rho y_{i}\left(1-y_{i}-a_{y, i} x_{i}\right)+\rho \beta_{y}\left(k_{y} y_{j}-y_{i}\right), \\
i, j=1,2, \quad i \neq j .
\end{gathered}
$$

The quantities $x_{i}$ and $y_{i}$ represent the densities of species 1 and 2 in the $i$ th patch scaled by their respective carrying capacities, and $a_{x, i}$ and $a_{y, i}$ represent the per capita effect of species 2 on species 1 (and vice versa) scaled by the ratio of respective carrying capacities. Quantities $k_{x}$ and $k_{y}$ represent the ratio of carrying capacities in the two patches for species 1 and 2 , respectively, $\beta_{x}$ and $\beta_{y}$ are the speciesspecific emigration rates scaled by their respective growth rates, $\rho$ is the ratio of the per capita growth rates of the two species, and $\tau$ is a time metric that is a composite of $t$ and $r_{x}$, the growth rate of species 1 . The dispersal scheme is such that individuals leaving one patch end up in the other patch, with no dispersal mortality in transit. This is equivalent to the island model of dispersal.

We are interested in a life-history trade-off between competitive and dispersal abilities. We describe such a trade-off in terms of competition coefficients (the per capita effect that a given species has on the other) and per capita dispersal rates. We assume the species and patches to be otherwise similar (i.e., $\rho=1, k_{x}=k_{y}=1$, $\left.K_{x, i}=K_{y, i}\right)$, which means that $a_{x, i}=\phi_{x, i}$ and $a_{y, i}=\phi_{y, i}$. This leads to the following simplified two-patch system:

$$
\begin{gathered}
\frac{d x_{i}}{d \tau}=x_{i}\left(1-x_{i}-\phi_{x, i} y_{i}\right)+\beta_{x}\left(x_{j}-x_{i}\right), \\
\frac{d y_{i}}{d \tau}=y_{i}\left(1-y_{i}-\phi_{y, i} x_{i}\right)+\beta_{y}\left(y_{j}-y_{i}\right), \\
i, j=1,2, \quad i \neq j .
\end{gathered}
$$


In the absence of dispersal $\left(\beta_{x}=\beta_{y}=0\right)$, competitive interactions within each patch lead to three basic outcomes (Volterra 1926; Lotka 1932): coexistence via niche partitioning $\left(\phi_{x, i}<1, \phi_{y, i}<1\right)$, exclusion via priority effects $\left(\phi_{x, i}>1, \phi_{y, i}>1\right)$, and exclusion via competitive asymmetry $\left(\phi_{x, i}<1, \phi_{y, i}>1\right.$, or vice versa). We focus exclusively on competitive asymmetry because this is the situation for which a life-history trade-off is most relevant.

When patches are linked by dispersal, spatial heterogeneity becomes an important consideration (Murdoch et al. 1992; Nisbet et al. 1993). We define spatial heterogeneity in terms of factors that affect the patch-specific competitive abilities of the two species $\phi_{x, i}$ and $\phi_{y, i}$. For instance, when $\phi_{x, i}=\phi_{x, j}<1$ and $\phi_{y, i}=\phi_{y, j}>1$ (or vice versa), the competitive environment is spatially homogeneous and one species is consistently superior within all patches of the landscape. When $\phi_{x, i} \neq \phi_{x, j}$ and $\phi_{y, i} \neq \phi_{y, j}$ (e.g., $\phi_{x, i}<1$, $\phi_{x, j}>1, \phi_{y, i}>1, \phi_{y, j}<1$, or vice versa), competitive rankings vary over space such that the species that is the superior competitor in some parts of the landscape is the inferior competitor in the other parts of the landscape. This type of spatial heterogeneity can arise due to intrinsic factors such as genetic variability or phenotypic plasticity in competitive ability (Huel and Huel 1996; Morrison and Molofsky 1999). It can also arise via extrinsic factors that affect the species differently. Examples include spatial variation in microclimatic factors, availability of a second, critical resource (Tilman and Pacala 1993), disturbances (Connell 1978), and keystone predation (Paine 1966).

We investigate the conditions under which a trade-off between competition and dispersal can lead to local coexistence of superior and inferior competitors. We focus on three specific situations, each motivated by empirical studies of dispersal-competition trade-offs.

\section{Local Coexistence When the Inferior Competitor Has a Refuge}

The first situation we analyze is motivated by Lei and Hanski's (1998) study of the parasitoids of Melitaea cinxia. Their data show a spatial pattern of local coexistence versus patches occupied only by the superior disperser (inferior competitor). Can local coexistence result from a dispersalcompetition trade-off?

We assume that the superior competitor does not move among occupied patches and is restricted to patch 1. Patch 2 is colonized solely by the inferior competitor and serves as a refuge from competition for that species. The inferior competitor also moves between the two patches at a rate $\beta_{y}$. This scenario leads to the following version of the model:

$$
\begin{aligned}
& \frac{d x_{1}}{d \tau}=x_{1}\left(1-x_{1}-\phi_{x, 1} y_{1}\right), \\
& \frac{d y_{1}}{d \tau}=y_{1}\left(1-y_{1}-\phi_{y, 1} x_{1}\right)+\beta_{y}\left(y_{2}-y_{1}\right), \\
& \frac{d y_{2}}{d \tau}=y_{2}\left(1-y_{2}\right)+\beta_{y}\left(y_{1}-y_{2}\right),
\end{aligned}
$$

with $x$ denoting the abundance of the superior, immobile competitor and $y$ denoting that of the inferior, mobile competitor. Note that $\phi_{x, 1}<1$ and $\phi_{y, 1}>1$.

In the absence of dispersal (i.e., $\beta_{y}=0$ ), patch 1 is a sink for the inferior competitor. This is because the superior competitor increases in abundance at the expense of the inferior competitor, preventing the latter from maintaining a positive growth rate.

Equation (4) yields four feasible equilibria: first, both species extinct $\left(x_{1}^{\star}, y_{1}^{\star}, y_{2}^{\star}\right)=(0,0,0)$; second, superior competitor at carrying capacity $(1,0,0)$; third, inferior competitor at carrying capacity $(0,1,1)$; and finally, the coexistence equilibrium.

Local coexistence of inferior and superior competitors requires that the inferior competitor be able to invade a patch when the superior competitor is at carrying capacity and that the coexistence equilibrium be stable to small perturbations in the abundance of both species.

We first investigate whether the inferior competitor can invade when rare in both patches. In appendix A, we show that invasion will succeed if

$$
\left(1-\phi_{y, 1}\right)-\beta_{y}\left(2-\phi_{y, 1}\right)<0 .
$$

The inferior competitor can invade when rare under two situations: if $\phi_{y, 1}<2$, invasion can occur as long as $\beta_{y}>0$, and if $\phi_{y, 1}>2$, then invasion is possible only as long as $\beta_{y}<\left(1-\phi_{y, 1}\right) /\left(2-\phi_{y, 1}\right)$ (fig. 1). Stability analyses (app. A) show that the coexistence equilibrium is stable when it exists.

The key result is that stable local coexistence of inferior and superior competitors can occur, but is not guaranteed, as long as there are patches in the landscape that are colonized only by the inferior competitor. Immigration from such refuge populations rescues the inferior competitor from exclusion in patches that are colonized by both species. Coexistence in the face of competitive asymmetry depends on both dispersal rates and degree of asymmetry. When competitive asymmetry in patch 1 is low (e.g., $\phi_{x, 1}<1$ and $1<\phi_{y, 1}<2$ ), coexistence occurs provided the inferior competitor has a nonzero dispersal rate. When competitive asymmetry is high (e.g., $\phi_{x, 1}<1$ and $\phi_{y, 1}>$ 2 ), coexistence occurs only as long as the dispersal rate is below a critical threshold. When the dispersal rate exceeds 


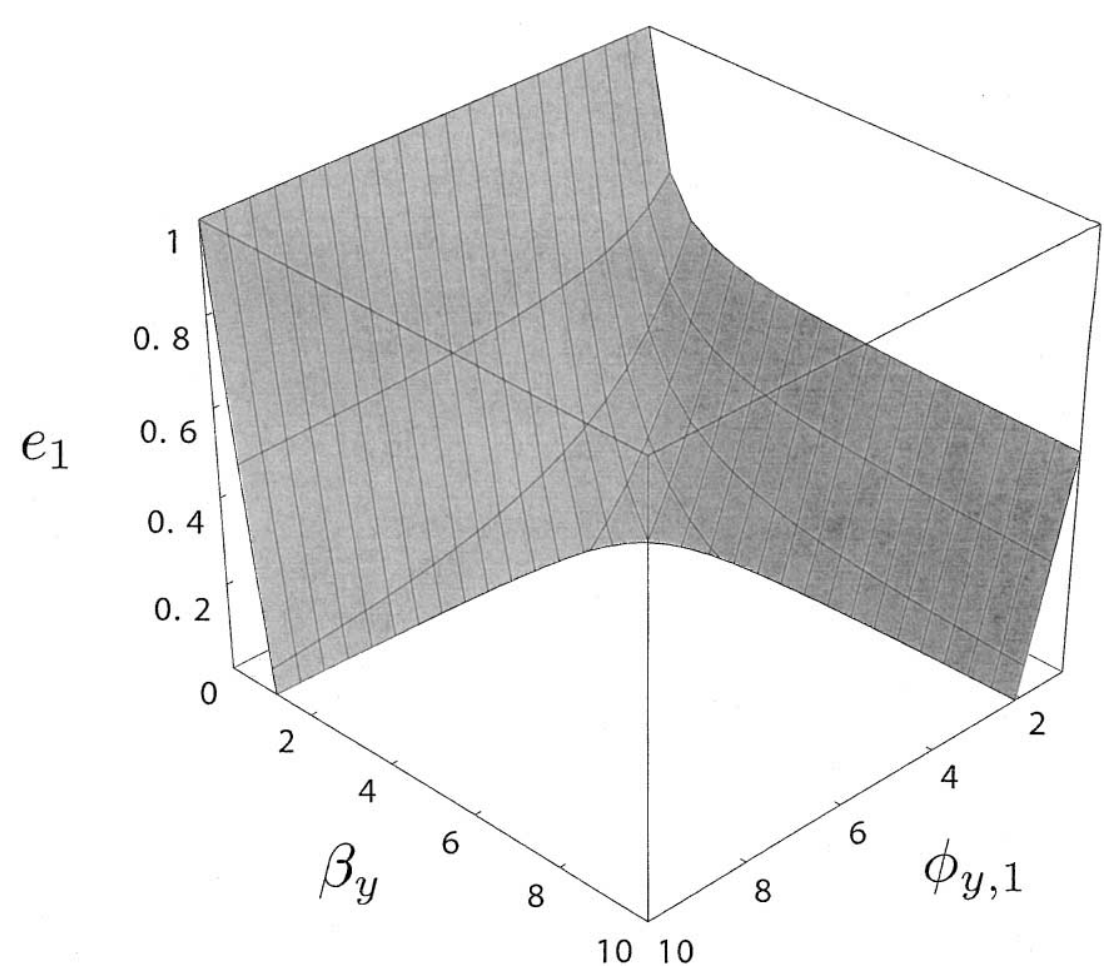

Figure 1: Conditions for invasion by the inferior competitor when it has a refuge (eq. [4]). The eigenvalue $e_{1}=\left[b+\left(b^{2}-4 c\right)^{1 / 2}\right] / 2(b$ and $c$ as defined in app. A) is plotted as a function of the competition coefficient $\left(\phi_{y, 1}\right)$ and dispersal rate $\left(\beta_{y}\right)$ of the inferior competitor. The surface depicts the portion of the parameter space where $e_{1}$ is positive (i.e., the inferior competitor can invade when rare). When $\phi_{y, 1}<2$, the invasion can occur as long as $\beta_{y}>0$. When $\phi_{y, 1}>2$, invasion is possible only as long as $\beta_{y}<\left[\left(1-\phi_{y, 1}\right) /\left(2-\phi_{y, 1}\right)\right]$. Note that $\beta_{y}$ is the ratio of per capita emigration to local growth (i.e., $\beta_{y}>1$ means that rate of emigration from the patch exceeds the local growth rate).

this threshold, net emigration from source to sink populations causes the source population growth rate to be negative, and the inferior competitor is excluded from the entire metapopulation.

\section{Local Coexistence When the Inferior Competitor Has No Refuge}

In Harrison et al.'s (1995) study of the herbivores of ragwort, no patches were found that were empty of the superior competitor. This suggests that the superior competitor has a colonization ability comparable to that of the inferior competitors. Our full two-patch model (eq. [3]) describes the situation where no refuges exist for the inferior competitor and both the superior and inferior competitors are able to move among occupied patches. Now the issue becomes more challenging: Can a superior ability to immigrate among occupied patches allow an inferior competitor to coexist locally with a superior competitor?

We first investigate whether the inferior competitor can invade when the superior competitor is at carrying capacity in both patches (i.e., $x_{1}^{\star}=x_{2}^{\star}=1$ ). In appendix B, we show that successful invasion requires $I<0$, where

$$
I=\left(1-\phi_{y, 1}\right)\left(1-\phi_{y, 2}\right)-\beta_{y}\left[\left(1-\phi_{y, 1}\right)+\left(1-\phi_{y, 2}\right)\right] .
$$

Note that the quantities $1-\phi_{y, 1}$ and $1-\phi_{y, 2}$ are the initial growth rates of the inferior competitor in patches 1 and 2 in the absence of dispersal (Pacala and Roughgarden 1982). Thus, the first term of I represents the product of the initial growth rates in the two patches and the second term their sum. The signs of these two quantities determine whether or not invasion can occur. For example, if the sum of the initial growth rates is positive and the product negative, $I<0$ as long as $\beta_{y}>0$. If both sum and product are negative, then whether or not $I<0$ depends on the actual magnitude of $\beta_{y}$.

We first consider the situation where the competitive environment is spatially homogeneous. When $\phi_{x, i}=$ $\phi_{x, j}=\phi_{x}<1$ and $\phi_{y, i}=\phi_{y, j}=\phi_{y}>1$, species 1 is the superior competitor across the metapopulation. Then the 
sum of the initial growth rates of the inferior competitor in the two patches is negative $\left(2-2 \phi_{y}<0\right)$ and the product positive $\left(\left[1-\phi_{y}\right]^{2}>0\right)$, which means that $I=(1-$ $\left.\phi_{y}\right)^{2}-\beta_{y}\left(2-2 \phi_{y}\right)>0$. The equilibrium with the superior competitor at carrying capacity cannot be invaded by the inferior competitor. Invasion fails because the superior competitor increases at the expense of the inferior competitor in both patches, causing the initial growth rate of the latter to be negative across the metapopulation.

The key result is that when the competitive environment is spatially homogeneous (i.e., one species is consistently the superior competitor), and when both species have comparable colonization abilities such that local refuges for the inferior competitor do not exist, a superior ability to migrate among occupied patches is not sufficient to prevent exclusion of the inferior competitor.

\section{The Role of Spatial Heterogeneity}

The above analysis shows that invasion fails in a competitively homogeneous environment because the inferior competitor has a negative initial growth rate in both patches. This suggests that invasion may succeed if the inferior competitor can maintain a positive initial growth rate in at least one patch. Mathematically, this means that the product of the initial growth rates in the two patches should be negative (i.e., $\left.\left[1-\phi_{y, 1}\right]\left[1-\phi_{y, 2}\right]<0\right)$. Since competition is assumed to be asymmetric (i.e., $\phi_{x, i}<1$ and $\phi_{y, i}>1$ or vice versa; $\left.i=1,2\right)$, the only way this can happen is if there is spatial heterogeneity in competitive rankings such that the superior competitor suffers a disadvantage in at least some parts of the landscape (e.g., $\left.\phi_{x, i}<1, \phi_{x, j}>1 ; i, j=1,2 ; i \neq j\right)$.

That inferior competitors can flourish in areas disadvantageous to superior competitors (e.g., keystone predation) is well known (Harper 1961; Paine 1966; Connell 1978; Lubchenco 1978). The novel issue we explore is whether dispersal from such areas allows the inferior competitor to persist in areas where the superior competitor itself flourishes. Because competitive rankings vary across space, the average competitive ability of each species becomes an important determinant of invasion and coexistence.

When the competitive environment is spatially heterogeneous, invasion can occur under three biologically distinct, and significant, circumstances. The first situation arises when competition is asymmetric at the scale of a local population but spatial averages of competition coefficients are such that niche partitioning occurs at the scale of the metapopulation. For instance, let $\phi_{x, 1}<1$, $\phi_{y, 1}>1$ in patch 1 and $\phi_{x, 2}>1, \phi_{y, 2}<1$ in patch 2 . Let the average competitive coefficients be $\bar{\phi}_{x}=\left[\left(\phi_{x, 1}+\right.\right.$ $\left.\left.\phi_{x, 2}\right) / 2\right]<1$ and $\bar{\phi}_{y}=\left[\left(\phi_{y, 1}+\phi_{y, 2}\right) / 2\right]<1$. Then, species 1 is the superior competitor in patch 1 and species 2 is the superior competitor in patch 2 , but neither species is superior in the sense that interspecific competition is weaker than intraspecific competition when averaged across the metapopulation. At the metapopulation scale, the two species meet the criteria for classical niche partitioning (Volterra 1926; Lotka 1932).

Under global niche partitioning, the sum of the initial growth rates is positive and the product negative, which means that $I<0$ as long as $\beta_{y}>0$. The equilibrium with the locally superior competitor at carrying capacity (i.e., $x_{1}^{\star}, x_{2}^{\star}, y_{1}^{\star}, y_{2}^{\star}=1,1,0,0$ or $\left.0,0,1,1\right)$ can be invaded by the locally inferior competitor (species 2 or 1 , respectively) as long as it has a nonzero dispersal rate $\left(\beta_{y}>0\right.$ or $\beta_{x}>0$, respectively).

The important point is that as long as competition is asymmetric locally and niche partitioning occurs globally, coexistence can occur even in the absence of a dispersalcompetition trade-off. The patch in which the species has local competitive superiority acts as a source of immigrants for the patch in which it is locally inferior. Thus, sourcesink dynamics allow each species to maintain small sink populations in areas of the landscape where it suffers a competitive disadvantage.

The second situation arises when competition is asymmetric both locally and globally. For example, species 1 is the superior competitor in patch $1\left(\phi_{x, 1}<1, \phi_{y, 1}>1\right)$ and species 2 is the superior competitor in patch $2\left(\phi_{x, 2}>1\right.$, $\left.\phi_{y, 2}<1\right)$, but now species 1 is the superior competitor when averaged across the metapopulation $\left(\bar{\phi}_{x}<1\right.$ and $\left.\bar{\phi}_{y}>1\right)$.

The species that is the overall superior competitor can invade when rare as long as it has a nonzero dispersal rate (i.e., $\beta_{x}>0$ ). The important issue is whether the overall inferior competitor can invade when rare. Global asymmetric competition means both the sum and the product of initial growth rates are negative. Invasibility now depends on the actual magnitude of $\beta_{y}$. Solving equation (6) for $\beta_{y}$ shows that the inferior competitor can invade only if its dispersal rate is below a critical threshold:

$$
\beta_{y}<\beta_{\text {critical }}=\frac{\left(1-\phi_{y, 1}\right)\left(1-\phi_{y, 2}\right)}{\left(1-\phi_{y, 1}\right)+\left(1-\phi_{y, 2}\right)}
$$

When competition is asymmetric both locally and globally, local coexistence does not involve a dispersal-competition trade-off. In fact, local coexistence requires that the dispersal rate of the overall inferior competitor not exceed a critical threshold. Once the dispersal rate exceeds this threshold, the overall inferior competitor cannot increase when rare even when it is competitively superior in some parts of the landscape.

The critical dispersal threshold depends on spatial het- 
erogeneity in competitive ability (fig. 2). The stronger the local competitive advantage to the overall inferior competitor in areas where the overall superior competitor is disadvantaged (e.g., $\phi_{y, i}>1, \phi_{y, j} \ll 1 \Rightarrow \bar{\phi}_{y} \rightarrow 1$ ), the larger the critical dispersal threshold and greater the possibility of local coexistence. If spatial heterogeneity in the environment is insufficient to create a strong local competitive advantage to the inferior competitor (e.g., $\phi_{y, i}>1, \phi_{y, j} \rightarrow$ $\left.1 \Rightarrow \bar{\phi}_{y}>1\right)$, then the threshold becomes correspondingly small and conditions for coexistence restrictive.

The key to coexistence, therefore, is spatial heterogeneity in competitive ability. There should be sufficient spatial variation in the biotic or abiotic environment that the overall superior competitor suffers a disadvantage in some parts of the landscape. Immigration from populations where the overall inferior competitor has a local advantage prevents its exclusion in areas where it has a local disadvantage. In contrast to global niche partitioning, however, coexistence is possible only as long as the dispersal rate of the overall inferior competitor is below a critical threshold. This is because individuals are moving from regions of the landscape where they are competitively superior and enjoy a positive growth rate (source populations) to regions where they are competitively inferior and suffer a negative growth rate (sink populations). If the net rate of emigration is sufficiently high relative to local reproduction that the growth rate of the source population becomes negative, the species loses its local competitive advantage and is excluded from the entire metapopulation.

So far, we have derived conditions for local coexistence for two situations: global niche partitioning and global competitive asymmetry. The third situation arises when

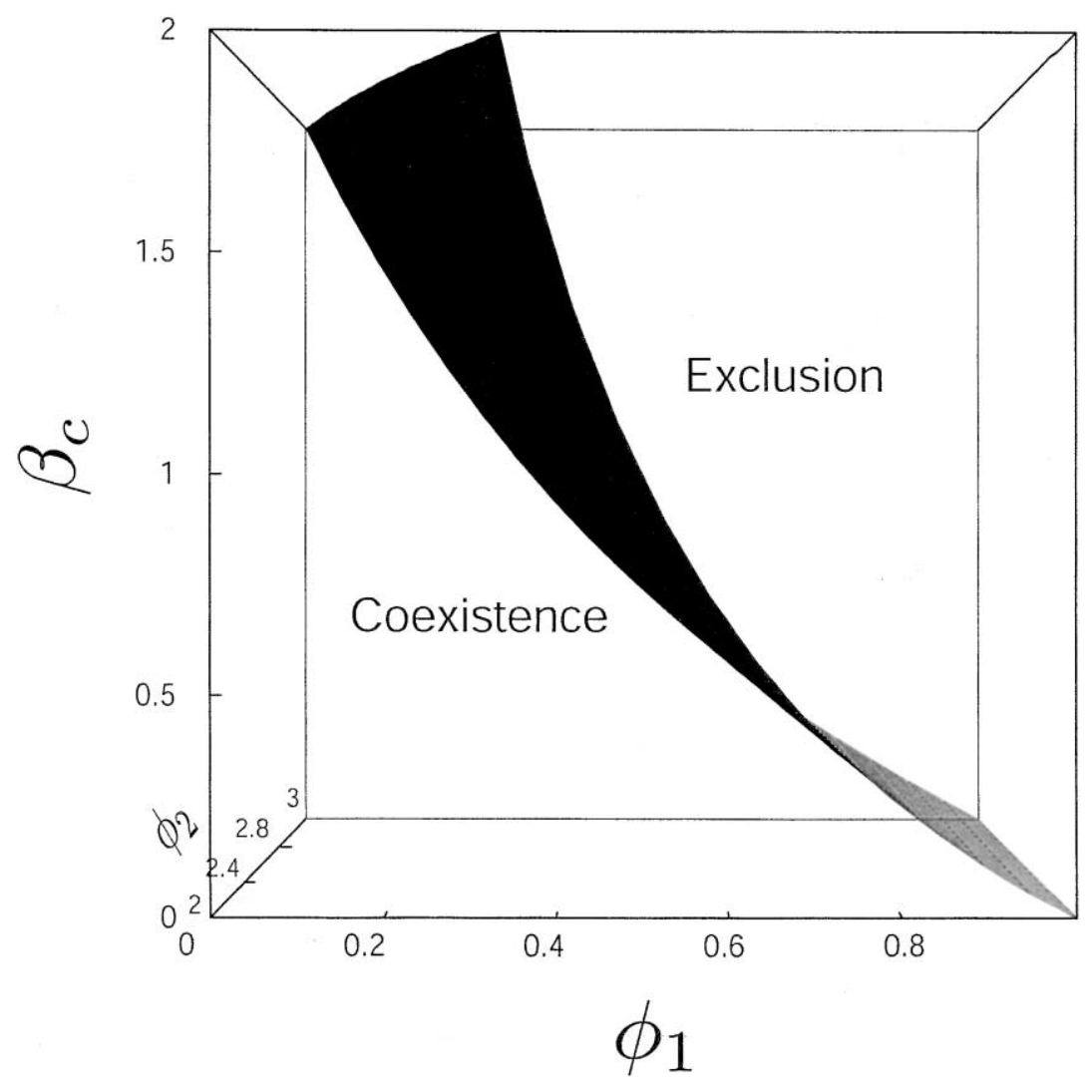

Figure 2: The relationship between spatial variance in competitive ability and the critical dispersal threshold $\left(\beta_{c}\right)$ when competition involves global asymmetry. The quantities $\phi_{1}$ and $\phi_{2}$ are the competition coefficients of the overall inferior competitor in patches 1 and 2, respectively. The overall inferior competitor enjoys a competitive advantage in patch $1\left(\phi_{1}<1\right)$ and suffers a competitive disadvantage in patch $2\left(\phi_{2}>1\right)$. Global asymmetry means that $\phi_{1}+\phi_{2}>2$ (or $\left.\bar{\phi}>1\right)$ for the inferior competitor. For any given value of $\phi_{2}, \beta_{c}$ reaches a maximum when $\phi_{1} \rightarrow 0\left(\lim _{\phi_{1} \rightarrow 0} \beta_{c}=[(1-\right.$ $\left.\left.\left.\phi_{2}\right) /\left(2-\phi_{2}\right)\right] ; \beta_{c}>0 \Rightarrow \phi_{2}>2\right)$ and a minimum when $\phi_{1} \rightarrow 1\left(\lim _{\phi_{1} \rightarrow 1} \beta_{c}=0\right)$. The larger $\phi_{2}$ is the smaller the maximum value of $\beta_{c}$ and steeper the decline of $\beta_{c}$ with $\phi_{1}$. In biological terms, this means that for any level of the competitive disadvantage suffered by the species in one patch, the stronger its local competitive advantage in the other patch, the higher the critical dispersal threshold, and hence, the possibility of coexistence. However, if the competitive disadvantage suffered by the species in one patch is large relative to its competitive advantage in the other patch, the dispersal threshold is lowered and conditions for coexistence become restrictive. 
competition is asymmetric locally but a priority effect occurs globally (i.e., species 1 is the superior competitor in patch $1\left[\phi_{x, 1}<1, \phi_{y, 1}>1\right]$, and species 2 is the superior competitor in patch $\left.2\left[\phi_{x, 2}>1, \phi_{y, 2}<1\right]\right)$, but interspecific competition is stronger than intraspecific competition when averaged across the metapopulation $\left(\bar{\phi}_{x}>1\right.$ and $\left.\bar{\phi}_{y}>1\right)$. Now each species has a critical dispersal threshold above which coexistence cannot occur:

$$
\beta_{.}<\beta_{\text {critical }}=\frac{\left(1-\phi_{\cdot 1}\right)\left(1-\phi_{\cdot 2}\right)}{\left(1-\phi_{\cdot 1}\right)+\left(1-\phi_{\cdot 2}\right)} .
$$

As with global asymmetry, the magnitude of the dispersal threshold depends on spatial heterogeneity in competitive ability (fig. 3). If the two species differ in the degree of local asymmetry but have the same competitive ability on average (e.g., $\phi_{x, 1} \neq \phi_{y, 2}, \phi_{x, 2} \neq \phi_{y, 1} ; \bar{\phi}_{x}=\bar{\phi}_{y}>1$ ), then local coexistence is determined by the dispersal ability of the species that experiences lower spatial heterogeneity and hence the lower dispersal threshold (fig. 3). If the species are sufficiently different that their average competition coefficients are unequal (e.g., $\bar{\phi}_{x}>1, \bar{\phi}_{y}>1$; $\left.\phi_{x}<\bar{\phi}_{y}\right)$, then local coexistence is determined by the dispersal ability of the species with the higher average competition coefficient (lower competitive ability). For instance, if $\bar{\phi}_{x}<\bar{\phi}_{y}$, then $\beta_{y_{\text {critical }}}<\beta_{x_{\text {critical }}}$, and the dispersal threshold for species 2 determines the transition from coexistence to exclusion.

The key to coexistence, again, is spatial heterogeneity in competitive ability. When heterogeneity is low $(\bar{\phi} .>1)$, the region of the parameter space where each species can invade when rare is small (fig. $3 B$ ); when heterogeneity is high $(\bar{\phi} . \rightarrow 1)$, this region is correspondingly larger (fig. 3C). An important difference between global asymmetry and a global priority effect is that while coexistence is determined by the dispersal ability of the overall inferior competitor in the former, dispersal abilities of both competing species determine conditions for coexistence in the latter. If both species have dispersal rates that exceed their respective thresholds, neither species can invade when rare and coexistence is impossible either locally or regionally.

These results lead to a set of comparative predictions (table 1). The three situations under which local coexistence can occur in a competitively heterogeneous environment can be distinguished by their response to the transition from low to high dispersal. In the absence of dispersal, all three situations exhibit global coexistence with each species flourishing in areas where it has a local competitive advantage. Under low dispersal, source-sink dynamics ensure local coexistence in all three cases. High dispersal, however, elicits qualitatively different dynamical responses. For instance, when competition involves global niche partitioning, local coexistence prevails. When competition involves global asymmetry, global exclusion of the overall inferior competitor results. When competition involves a global priority effect, the outcome is global exclusion of the species with the lower dispersal threshold.

Another key distinction between the three situations is the relative sensitivity of competing species to a perturbation that increases dispersal between patches. Neither species is sensitive to such a perturbation under global niche partitioning, only the inferior competitor is sensitive under global asymmetry, and both species are sensitive under a global priority effect. In other words, there are no constraints on the dispersal abilities when global niche partitioning occurs, while constraints exist on one or both species when global asymmetry or priority effects occur. This suggests that species that experience local competitive asymmetry but partition niches globally have the highest likelihood of coexistence, while those that experience global priority effects have the least.

\section{Discussion}

This study was motivated by empirical investigations of dispersal-competition trade-offs in insect systems. Observations of local coexistence in these studies are at odds with the prediction of regional coexistence from patch occupancy theory. These observations suggest that dispersal may be sufficiently rapid to counteract competitive exclusion, a possibility that cannot be addressed in the patch occupancy framework because of the decoupling of local and spatial dynamics. We developed models that explicitly consider local dynamics in which spatial processes of emigration and immigration operate on the same time scale as local competitive interactions. Our objective was to determine the conditions under which the interaction between competition and dispersal could lead to local coexistence.

Our findings provide potential explanations for the patterns seen in several insect systems. For instance, in Lei and Hanski's (1998) study of the parasitoids of Melitaea cinxia, the inferior competitor is found in patches that are not colonized by the superior competitor, suggesting that it has a superior colonization ability. The observed pattern of local coexistence in some patches versus the inferior competitor by itself in other patches could arise if immigration from the latter prevents competitive exclusion in the former.

In contrast to the butterfly system, however, the two parasitoids of the harlequin bug have comparable colonization abilities (Amarasekare 2000a). In such a situation, immigration could counteract competitive exclusion if there is spatial variance in competitive rankings. However, one parasitoid species is consistently the superior com- 

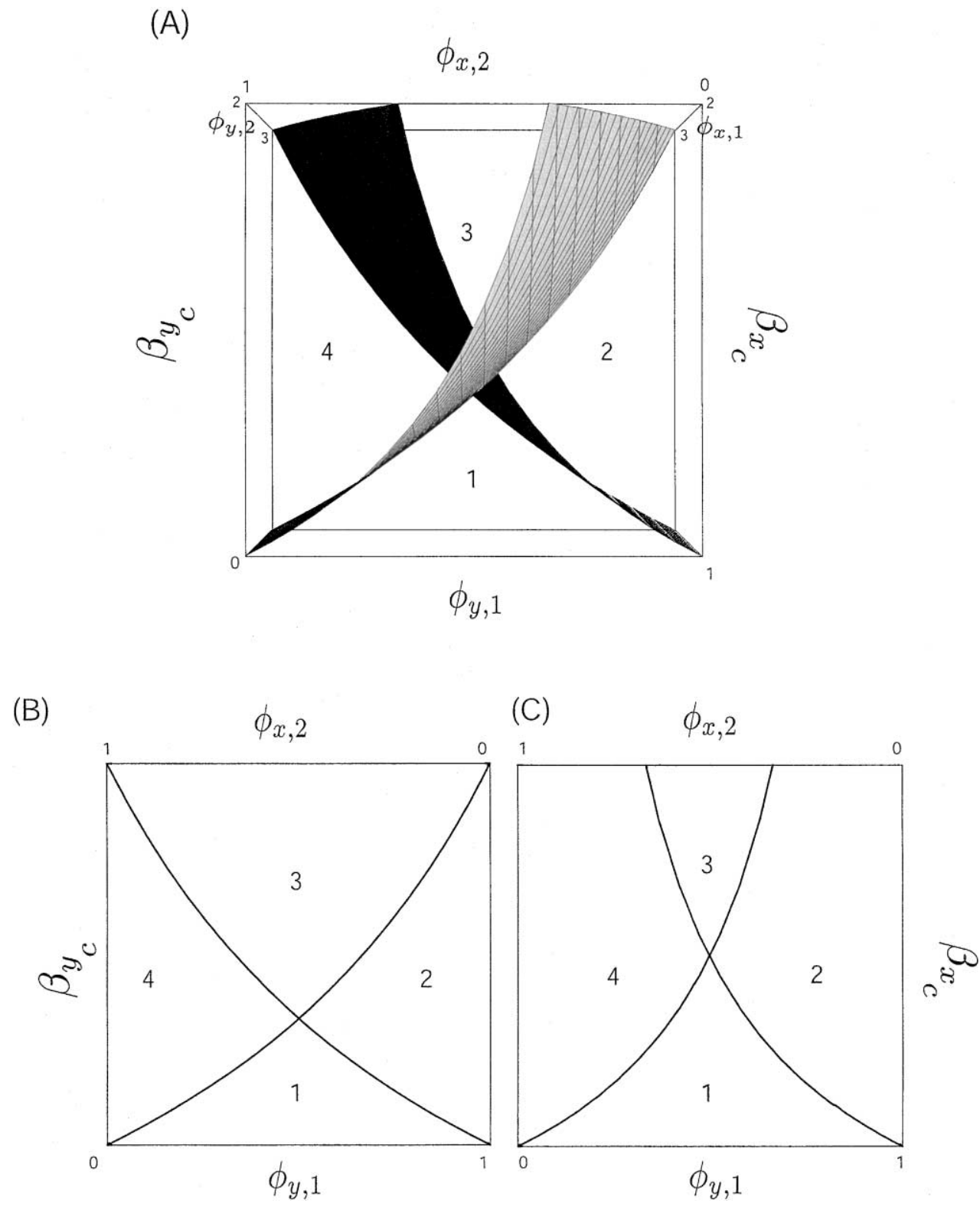

Figure 3: The relationship between spatial variance in competitive ability and the critical dispersal threshold when competition involves a global priority effect. $A$, The three-dimensional parameter space with the critical dispersal threshold for each species as a function of its competitive coefficients in the two patches. The black surface depicts this relationship for species 2 and the gray surface for species 1 . Note that the $X$-axis goes from 1 to 0 for species 1 . B, C, Two-dimensional slices of the parameter space for $\phi_{x, 1}=\phi_{y, 2}=3$ and $\phi_{x, 1}=\phi_{y, 2}=2$, respectively. Region 1 represents the portion of the parameter space where both species can invade when the other species is at carrying capacity. The long-term outcome is stable coexistence. Region 2 represents the parameter space where species 2 cannot invade when rare, and region 4, where species 1 cannot invade when rare. The long-term outcome is exclusion of species 2 and 1, respectively. Region 3 represents the parameter space where neither species can invade when rare. The long-term outcome is global exclusion of the species that exhibits the lower dispersal threshold. When spatial heterogeneity is low (i.e., local competitive advantage enjoyed by a species in one patch is small relative to the disadvantage it suffers in the other patch), the region where both species can invade is small and the region where neither species can invade is large $(B)$. When spatial heterogeneity is high, the region of mutual invasibility increases relative to the region of mutual noninvasibility $(C)$. 
Table 1: Comparative predictions for invasion and local coexistence under different regimes of spatial heterogeneity

\begin{tabular}{|c|c|c|c|}
\hline $\begin{array}{l}\text { Competitive } \\
\text { environment }\end{array}$ & Isolation & Low dispersal & High dispersal \\
\hline $\begin{array}{l}\text { Local asymmetry, global niche } \\
\text { partitioning }\end{array}$ & $\begin{array}{l}\text { Locally inferior competitor } \\
\text { cannot invade } \\
\text { Global coexistence } \\
\beta_{x}, \beta_{y}=0\end{array}$ & $\begin{array}{l}\text { Both species can invade } \\
\text { Local coexistence } \\
\beta_{x}, \beta_{y}>0\end{array}$ & $\begin{array}{l}\text { Both species can invade } \\
\text { Local coexistence } \\
\beta_{x}, \beta_{y}>0\end{array}$ \\
\hline $\begin{array}{l}\text { Local asymmetry, } \\
\text { global asymmetry }\end{array}$ & $\begin{array}{l}\text { Locally inferior competitor } \\
\text { cannot invade } \\
\text { Global coexistence } \\
\beta_{x}, \beta_{y}=0\end{array}$ & $\begin{array}{l}\text { Both species can invade } \\
\text { Local coexistence } \\
0<\beta_{y}<\beta_{\text {critical }}, \beta_{x}>0\end{array}$ & $\begin{array}{l}\text { Overall inferior } \\
\text { competitor cannot invade } \mathrm{a}^{\mathrm{a}} \\
\text { Global exclusion of inferior competitor } \\
\beta_{y}>\beta_{\text {critical }}, \beta_{x}>0\end{array}$ \\
\hline $\begin{array}{l}\text { Local asymmetry, global priority } \\
\text { effect }\end{array}$ & $\begin{array}{l}\text { Locally inferior competitor } \\
\text { cannot invade } \\
\text { Global coexistence } \\
\beta_{x}, \beta_{y}=0\end{array}$ & $\begin{array}{l}\text { Both species can invade } \\
\text { Local coexistence } \\
0<\beta_{x}, \beta_{y}<\beta_{\text {critical }}\end{array}$ & $\begin{array}{l}\text { Neither species can invade }{ }^{\mathrm{b}} \\
\text { Global exclusion of species with lower } \\
\text { dispersal threshold } \\
\beta_{x}, \beta_{y}>\beta_{\text {critical }}\end{array}$ \\
\hline
\end{tabular}

a Transition from low to high dispersal affects inferior competitor only.

b Transition from low to high dispersal affects both species.

petitor all across the landscape, and experimental manipulations of dispersal rates have no effect on patterns of local coexistence (Amarasekare 2000a, 2000b). This study exemplifies a situation where lack of spatial variance in competitive ability precludes coexistence via source-sink dynamics.

Harrison et al.'s (1995) study of the herbivores of ragwort (Senecio jacobea) illustrates how rapid dispersal and spatial variance in competitive ability may counteract local exclusion. At the authors' study site in Silwood Park, Ascot, Berkshire, United Kingdom, the cinnabar moth (Tyria jacobeae) is the superior competitor and depresses the abundances of the flower head-feeding fly Botanophila seneciella and the flower galler Contarina jacobeae. However, local competitive exclusion is not observed even in patches heavily defoliated by the moth. Cinnabar moths are sufficiently mobile so that no ragwort patches exist that are empty of the moths. There is, however, spatial variation in cinnabar moth performance; the moth consistently defoliates dense patches of ragwort but performs consistently less well in patches that are heavily mown or in which the ragwort is sparsely distributed. The authors suggest that the patches of sparse ragwort may serve as refuges for $B$. seneciella and $C$. jacobeae. Both species have good dispersal abilities (B. seneciella is in fact a faster disperser than the cinnabar moth) and are able to invade areas where ragwort has been extensively defoliated. This suggests that immigration from patches where the moth performs poorly may be counteracting competitive exclusion of $B$. seneciella and C. jacobeae in patches where defoliation by the moth is extensive.

While there is certainly a potential for spatial dynamics to counteract competitive exclusion, we frequently observe instances of region-wide competitive displacement. The displacement of the native asexual gecko Lepidodactylus lugubris by the introduced sexual species Hemidactylus frenatus in the tropical Pacific (Case et al. 1994; Petren and Case 1996) and that of Aphytis lingnanensis, a parasitoid of the red scale (Aonidiella aurantii), by its congener Aphytis melinus in southern California (Luck and Podoler 1985; Murdoch et al. 1996) provide some of the more well studied examples. These observations suggest that, in some cases at least, spatial processes cannot counteract competitive exclusion because of insufficient spatial variance in competitive ability. If one species is competitively superior and is sufficiently mobile so that no refuges for the inferior competitor exist, then all local populations are essentially sinks for the inferior competitor. Dispersal from one location to another cannot prevent competitive exclusion. Typically, one would expect such large-scale exclusion to occur when competitive ability is determined by traits that are fixed within and across populations. For example, A. melinus gains a competitive advantage over A. lingnanensis because of a life-history difference; A. melinus is able to obtain female offspring from a smaller-sized scale than A. lingnanensis (Luck and Podoler 1985). Using a stage-structured host-parasitoid model, Murdoch et al. (1996) showed that this subtle difference is sufficient to explain the rapid displacement of A. lingnanensis from inland areas of southern California. If, however, the lifehistory traits influencing competitive ability are plastic, or if competitive ability is determined by extrinsic factors that are either biotic (predators, parasites, and pathogens) or abiotic (temperature, humidity, and disturbances), then 
spatial variation in these factors could provide conditions under which source-sink dynamics can lead to local coexistence. One would expect global niche partitioning to provide the broadest conditions for coexistence because this situation imposes no constraints on dispersal ability. Field measurements required to quantify the nature of the competitive environment include any measure of fitness or competitive ability such as growth rates or competitive coefficients and some measure of dispersal either through direct mark-recapture experiments or indirectly by using genetic markers (e.g., Dias 1996).

In our models, the interaction between competition and dispersal leads to a threshold effect. Levin (1974) documented a similar phenomenon for competition involving local priority effects. Since local dynamics themselves involve a threshold phenomenon in Levin's model, perhaps it is not surprising that this effect should persist when local populations are linked by dispersal. However, the models we have analyzed involve asymmetric competition that leads to deterministic extinction of inferior competitors in the absence of ameliorating forces. A threshold effect arises in our models when patches are coupled by dispersal, but only when competition involves global asymmetry or a global priority effect. A similar effect has been observed in some population genetic models as well. For instance, in Slatkin's (1994) model of epistatic, directional selection for complex adaptive traits, fixation of the adaptive genotype occurs in the absence of gene flow. With small amounts of gene flow, a stable polymorphism results with the less adaptive genotypes being maintained in the population. With large amounts of gene flow, the polymorphism disappears and the system reverts to monomorphism. The same dynamical phenomenon is observed in the classical migration-selection models with directional selection for a recessive allele (Wright 1931, 1969; Hartl and Clark 1997). A polymorphism arises under small amounts of gene flow and disappears under large amounts of gene flow. In contrast, competition-dispersal balance with global niche partitioning (this study) or migrationselection balance with directional selection for a dominant allele (Wright 1931, 1969; Hartl and Clark 1997) do not lead to threshold phenomena. In these cases, the transition from weak to strong coupling has no impact on coexistence.

A threshold effect resulting from the interaction between competition and dispersal has also been observed in a diffusion model (Pacala and Roughgarden 1982), suggesting that the phenomenon may operate in both patchy and spatially continuous environments. Pacala and Roughgarden investigated the role of habitat suitability in the invasion success of competing species. They modeled habitat suitability in terms of spatially varying carrying capacities and derived critical dispersal thresholds for a diffusion model as well as a discrete compartment analogue. They found that invasion could sometimes succeed when both sides of the environment were unsuitable and that it could sometimes fail when both sides of the environment were suitable. The authors attribute this result to the cost incurred when dispersal interacts directly with local population dynamics (i.e., individuals are moving from areas of higher to lower fitness). In Pacala and Roughgarden's (1982) model, as in ours, dispersal is costly because it is random. If dispersal were directional or density dependent, then conclusions about competitive coexistence might be different from what we have obtained above. In fact, density-dependent dispersal may be sufficiently nonlinear to counteract competitive exclusion even in a competitively homogeneous environment. This possibility remains to be explored.

In summary, explicit consideration of the interaction between competition and dispersal takes us away from the patch occupancy framework with its emphasis on extinction-colonization dynamics to the realm of source-sink dynamics. The key to coexistence in this framework is spatial variance in fitness. Unlike in the patch occupancy framework where local and spatial dynamics are essentially decoupled, high rates of dispersal can undermine coexistence, and hence diversity, by reducing spatial variance in fitness.

\section{Acknowledgments}

This research was supported by a postdoctoral fellowship to P.A. by the National Center for Ecological Analysis and Synthesis (a center funded by the National Science Foundation [DEB-9421535]), the University of California, Santa Barbara, the state of California, and by grant N000149910024 to R.M.N. from the Office of Naval Research. We thank J. Bascompte, O. Bjornstad, and two anonymous reviewers for helpful comments on the manuscript.

\section{APPENDIX A}

Invasion and Stability Analyses for the Model with a Refuge for the Inferior Competitor

The Jacobian matrix of equation (4) is given by 


$$
\left[\begin{array}{lll}
1-2 x_{1}^{\star}-\phi_{x, 1} y_{1}^{\star} & -\phi_{x, 1} x_{1}^{\star} & 0 \\
-\phi_{y, 1} y_{1}^{\star} & 1-2 y_{1}^{\star}-\phi_{y, 1} x_{1}^{\star}-\beta_{y} & \beta_{y} \\
0 & \beta_{y} & 1-2 y_{2}^{\star}-\beta_{y}
\end{array}\right] .
$$

Note that the Jacobian is a tridiagonal, quasi-symmetric matrix (i.e., all nonzero off-diagonal elements $A_{i, i+1}$ and $A_{i+1, i}$ have the same sign [Wilkinson 1965]). All eigenvalues of a quasi-symmetric matrix are real (see Wilkinson 1965, p. 336). This means that the transition from stability to instability involves a zero real root rather than a complex root with zero real parts. Hence, the system defined by equation (4) does not exhibit oscillatory instability (Gurney and Nisbet 1998).

The inferior competitor can invade when the superior competitor is at carrying capacity if the dominant eigenvalue of the Jacobian is positive when evaluated at $\left(x_{1}^{\star}, y_{1}^{\star}, y_{2}^{\star}\right)=(1,0,0)$.

The eigenvalues are -1 and $\left[b \pm\left(b^{2}-4 c\right)^{1 / 2}\right] / 2$, where $b=\left(1-\phi_{y, 1}-\beta_{y}\right)$ and $c=\left(1-\phi_{y, 1}-\beta_{y}\right)\left(1-\beta_{y}\right)-\beta_{y}^{2}$. The equilibrium $\left(x_{1}^{\star}, y_{1}^{\star}, y_{2}^{\star}\right)=(1,0,0)$ is unstable to invasion by the inferior competitor if the eigenvalue $[b+$ $\left.\left(b^{2}-4 c\right)^{1 / 2} / 2\right]>0$. This occurs when $c<0$ and leads to the invasion criterion (eq. [5]) in main text.

The characteristic equation for the Jacobian of equation (4) is:

$$
\lambda^{3}+A_{1} \lambda^{2}+A_{2} \lambda+A_{3}=0
$$

where

$$
\begin{aligned}
A_{1}= & -\left(1-2 x_{1}^{\star}-\phi_{x, 1} y_{1}^{\star}\right)-\left(1-2 y_{1}^{\star}-\phi_{y, 1} x_{1}^{\star}-\beta_{y}\right)-\left(1-2 y_{2}^{\star}-\beta_{y}\right), \\
A_{2}= & \left(1-2 y_{1}^{\star}-\phi_{y, 1} x_{1}^{\star}-\beta_{y}\right)\left[\left(1-2 x_{1}^{\star}-\phi_{x, 1} y_{1}^{\star}\right)+\left(1-2 y_{2}^{\star}-\beta_{y}\right)\right] \\
& +\left(1-2 x_{1}^{\star}-\phi_{x, 1} y_{1}^{\star}\right)\left(1-2 y_{2}^{\star}-\beta_{y}\right)-\phi_{x, 1} x_{1}^{\star} \phi_{y, 1} y_{1}^{\star}-\beta_{y}^{2},
\end{aligned}
$$

and

$A_{3}=\left(1-2 x_{1}^{\star}-\phi_{x, 1} y_{1}^{\star}\right) \beta_{y}^{2}+\phi_{x, 1} x_{1}^{\star} \phi_{y, 1} y_{1}^{\star}\left(1-2 y_{2}^{\star}-\beta_{y}\right)-\left(1-2 x_{1}^{\star}-\phi_{x, 1} y_{1}^{\star}\right)\left(1-2 y_{1}^{\star}-\phi_{y, 1} x_{1}^{\star}-\beta_{y}\right)\left(1-2 y_{2}^{\star}-\beta_{y}\right)$.

We were unable to derive a rigorous proof of local stability of the coexistence equilibrium. As the roots of the characteristic equation are real, stability is guaranteed if $A_{1}>0, A_{2}>0$, and $A_{3}>0$. It is easy to show that $A_{1}$ is positive, so proving stability involves proving positivity of $A_{2}$ and $A_{3}$. We obtained a convincing numerical demonstration that these coefficients are positive by noting that the model has only three parameters $\left(\beta_{y}, \phi_{x, 1}\right.$, and $\left.\phi_{y, 1}\right)$ that are related to the equilibrium conditions by the equations:

$$
\begin{aligned}
\beta_{y} & =\frac{y_{2}^{\star}\left(1-y_{2}^{\star}\right)}{y_{2}^{\star}-y_{1}^{\star}}, \\
\phi_{x, 1} & =\frac{1-x_{1}^{\star}}{y_{1}^{\star}}, \\
\phi_{y, 1} & =\frac{1-y_{1}^{\star}}{x_{1}^{\star}}+\frac{y_{2}^{\star}\left(1-y_{2}^{\star}\right)}{x_{1}^{\star} y_{1}^{\star}} .
\end{aligned}
$$

The scaled equilibrium populations are restricted to the range $(0,1)$, so we divided the unit cube in three-dimensional $\left(x_{1}^{\star}, y_{1}^{\star}, y_{2}^{\star}\right)$ space into a fine grid (intervals of 0.005 in each variable) and evaluated $A_{2}$ and $A_{3}$ for all points $\left(x_{1}^{\star}, y_{1}^{\star}\right.$, $\left.y_{2}^{\star}\right)$ that yielded $\phi_{x, 1}<1, \phi_{y, 1}>1$, and $\beta_{y}>0$. No unstable equilibria were found.

\section{APPENDIX B}

Invasion and Stability Analyses for the Model with No Refuge for the Inferior Competitor

The Jacobian matrix for equation (3) is: 


$$
\left[\begin{array}{llll}
1-2 x_{1}^{\star}-\phi_{x, 1} y_{1}^{\star}-\beta_{x} & \beta_{x} & -\phi_{x, 1} x_{1}^{\star} & 0 \\
\beta_{x} & 1-2 x_{2}^{\star}-\phi_{x, 2} y_{2}^{\star}-\beta_{x} & 0 & -\phi_{x, 2} x_{2}^{\star} \\
-\phi_{y, 1} y_{1}^{\star} & 0 & 1-2 y_{1}^{\star}-\phi_{y, 1} x_{1}^{\star}-\beta_{y} & \beta_{y} \\
0 & -\phi_{y, 2} y_{2}^{\star} & \beta_{y} & 1-2 y_{2}^{\star}-\phi_{y, 2} x_{2}^{\star}-\beta_{y}
\end{array}\right] .
$$

The Jacobian is a quasi-symmetric matrix. All eigenvalues are therefore real, and hence the system defined by equation (3) does not exhibit oscillatory instability (Gurney and Nisbet 1998).

The inferior competitor can invade when rare in both patches if the dominant eigenvalue of the Jacobian is positive when evaluated at $\left(x_{1}^{\star}, x_{2}^{\star}, y_{1}^{\star}, y_{2}^{\star}\right)=(1,1,0,0)$. The eigenvalues are $-1,-1-2 \beta_{y}$, and $\left[b \pm\left(b^{2}-4 c\right)^{1 / 2}\right] / 2$, where $b=\left(1-\phi_{y, 1}-\beta_{y}\right)+\left(1-\phi_{y, 2}-\beta_{y}\right)$ and $c=\left(1-\phi_{y, 1}-\beta_{y}\right)\left(1-\phi_{y, 2}-\beta_{y}\right)-\beta_{y}^{2}$. The equilibrium $\left(x_{1}^{\star}, x_{2}^{\star}, y_{1}^{\star}, y_{2}^{\star}\right)=$ $(1,1,0,0)$ is unstable to invasion by the inferior competitor if the eigenvalue $\left[b+\left(b^{2}-4 c\right)^{1 / 2}\right] / 2>0$. This leads to the invasion criterion (eq. [6]) in the main text.

Local stability criteria for the coexistence equilibrium cannot be derived analytically. Numerical explorations over the parameter range $\phi_{x, 1}=(0,1), \phi_{y, 1}=(1,5), \phi_{x, 2}=(1,5), \phi_{y, 2}=(0,1), \beta_{x}=(0,5), \beta_{y}=(0,5)$ show all four eigenvalues to be negative for all positive values of the coexistence equilibrium.

\section{Literature Cited}

Amarasekare, P. 2000a. Spatial dynamics in a host-multiparasitoid community. Journal of Animal Ecology 69: 201-213.

- 2000b. Coexistence of competing parasitoids on a patchily distributed host: local vs. spatial mechanisms. Ecology 81:1286-1296.

Case, T. J., D. T. Bolger, and K. Petren. 1994. Invasions and competitive displacement among house geckos in the tropical Pacific. Ecology 75:464-477.

Cohen, J. E. 1970. A Markov contingency-table model for replicated Lotka-Volterra systems near equilibrium. American Naturalist 104:547-560.

Connell, J. H. 1978. Diversity in tropical rainforests and coral reefs. Science (Washington, D.C.) 199:1302-1310.

Dias, P. C. 1996. Sources and sinks in population biology. Trends in Ecology \& Evolution 11:326-330.

Gurney, W. S. C., and R. M. Nisbet. 1998. Ecological dynamics. Oxford University Press, New York.

Harper, J. L. 1961. Approaches to the study of plant competition. Pages 1-39 in F. L. Milthrope, ed. Mechanisms in biological competition. Society for Experimental Biology Symposium 15. Cambridge University Press, Cambridge.

Harrison, S., C. D. Thomas, and T. M. Lewinsohn. 1995. Testing a metapopulation model of coexistence in the insect community of ragwort (Senecio jacobea). American Naturalist 145:546-562.

Hartl, D. L., and A. G. Clark. 1997. Principles of population genetics. Sinauer, Sunderland, Mass.

Hastings, A. 1980. Disturbance, coexistence, history and competition for space. Theoretical Population Biology 18:363-373.

Huel, D. G., and P. Huel. 1996. Genotypic variation for competitive ability in spring wheat. Plant Breeding 115: 325-329.

Lei, G., and I. Hanski. 1998. Spatial dynamics of two competing specialist parasitoids in a host metapopulation. Journal of Animal Ecology 67:422-433.

Levin, S. A. 1974. Dispersion and population interactions. American Naturalist 108:207-228.

Levins, R. 1969. Some demographic and genetic consequences of environmental heterogeneity for biological control. Bulletin of the Entomological Society of America 15:237-240.

. 1970. Extinction. Pages 75-107 in M. Gerstenhaber, ed. Some mathematical problems in biology. American Mathematical Society, Providence, R.I.

Levins, R., and D. Culver. 1971. Regional coexistence of species and competition between rare species. Proceedings of the National Academy of Sciences of the USA 68:1246-1248.

Lotka, A. J. 1932. The growth of mixed populations: two species competing for a common food supply. Journal of the Washington Academy of Sciences 22:461-469.

Lubchenco, J. 1978. Plant species diversity in a marine intertidal community: importance of herbivore food performance and algal competitive abilities. American Naturalist 112:23-39.

Luck, R. F., and H. Podoler. 1985. Competitive exclusion of Aphytis lingnanensis by Aphytis melinus: potential role of host size. Ecology 66:904-913.

Morrison, S. L., and J. Molofsky. 1999. Environmental and genetic effects on the early survival and growth of the invasive grass Phalaris arundinacea. Canadian Journal of Botany 77:1447-1453.

Murdoch, W. W., C. J. Briggs, R. M. Nisbet, W. S. C. Gurney, and A. Stewart-Oaten. 1992. Aggregation and stability in metapopulation models. American Naturalist 140:41-58. 
Murdoch, W. W., C. J. Briggs, and R. M. Nisbet. 1996. Competitive displacement and biological control in parasitoids: a model. American Naturalist 148:807-826.

Murray, J. D. 1993. Mathematical biology. Springer, New York.

Nee, S., and R. M. May. 1992. Dynamics of metapopulations: habitat destruction and competitive coexistence. Journal of Animal Ecology 61:37-40.

Nisbet, R. M., C. J. Briggs, W. S. C. Gurney, W. W. Murdoch, and A. Stewart-Oaten. 1993. Two-patch metapopulation dynamics. Pages 125-135 in S. A. Levin, T. Powell, and J. Steele, eds. Patch dynamics in freshwater, terrestrial and aquatic ecosystems. Springer, Berlin.

Pacala, S. W., and J. Roughgarden. 1982. Spatial heterogeneity and interspecific competition. Theoretical Population Biology 21:92-113.

Paine, R. T. 1966. Food web complexity and species diversity. American Naturalist 100:65-75.

Petren, K., and T. J. Case. 1996. An experimental demonstration of exploitation competition in an ongoing invasion. Ecology 77:118-132.

Skellam, J. G. 1951. Random dispersal in theoretical populations. Biometrika 38:196-218.

Slatkin, M. 1974. Competition and regional coexistence. Ecology 55:128-134.
1994. Epistatic selection opposed by migration in multiple locus genetic systems. Journal of Evolutionary Biology 8:623-633.

Tilman, D., and S. Pacala. 1993. The maintenance of species richness in plant communities. Pages 13-25 in R. Ricklefs and D. Schluter, eds. Species diversity in ecological communities. University of Chicago Press, Chicago.

Tilman, D., R. M. May, C. L. Lehman, and M. A. Nowak. 1994. Habitat destruction and the extinction debt. Nature 371:65-66.

Volterra, V. 1926. Variations and fluctuations of the numbers of individuals in animal species living together. Pages 409-448 in R. N. Chapman, ed. Animal ecology. McGraw-Hill, New York.

Wilkinson, J. H. 1965. The algebraic eigenvalue problem. Clarendon, Oxford.

Wright, S. 1931. Evolution in Mendelian populations. Genetics 16:97-159.

. 1969. Evolution and the genetics of populations. Vol. 2. The theory of gene frequencies. University of Chicago Press, Chicago. 


\title{
Spatial variation and density-dependent dispersal in competitive coexistence
}

\author{
Priyanga Amarasekare \\ Department of Ecology and Evolution, The University of Chicago, 1101 East 57th Street, Chicago, IL 60637, USA \\ (amarasek@uchicago.edu)
}

\begin{abstract}
It is well known that dispersal from localities favourable to a species' growth and reproduction (sources) can prevent competitive exclusion in unfavourable localities (sinks). What is perhaps less well known is that too much emigration can undermine the viability of sources and cause regional competitive exclusion. Here, I investigate two biological mechanisms that reduce the cost of dispersal to source communities. The first involves increasing the spatial variation in the strength of competition such that sources can withstand high rates of emigration; the second involves reducing emigration from sources via density-dependent dispersal. I compare how different forms of spatial variation and modes of dispersal influence source viability, and hence source-sink coexistence, under dominance and pre-emptive competition. A key finding is that, while spatial variation substantially reduces dispersal costs under both types of competition, density-dependent dispersal does so only under dominance competition. For instance, when spatial variation in the strength of competition is high, coexistence is possible (regardless of the type of competition) even when sources experience high emigration rates; when spatial variation is low, coexistence is restricted even under low emigration rates. Under dominance competition, density-dependent dispersal has a strong effect on coexistence. For instance, when the emigration rate increases with density at an accelerating rate (Type III densitydependent dispersal), coexistence is possible even when spatial variation is quite low; when the emigration rate increases with density at a decelerating rate (Type II density-dependent dispersal), coexistence is restricted even when spatial variation is quite high. Under pre-emptive competition, density-dependent dispersal has only a marginal effect on coexistence. Thus, the diversity-reducing effects of high dispersal rates persist under pre-emptive competition even when dispersal is density dependent, but can be significantly mitigated under dominance competition if density-dependent dispersal is Type III rather than Type II. These results lead to testable predictions about source-sink coexistence under different regimes of competition, spatial variation and dispersal. They identify situations in which density-independent dispersal provides a reasonable approximation to species' dispersal patterns, and those under which consideration of density-dependent dispersal is crucial to predicting long-term coexistence.
\end{abstract}

Keywords: competition; density-dependent dispersal; dominance; pre-emption; spatial variation; source-sink dynamics

\section{INTRODUCTION}

It is well known that dispersal can allow species coexistence if spatial variation in the relative strengths of intraspecific and interspecific interactions creates a source-sink population structure (e.g. Levin 1974; Pacala \& Roughgarden 1982; Shmida \& Ellner 1984; Kishimoto 1990; Amarasekare \& Nisbet 2001; Codeco \& Grover 2001; Mouquet \& Loreau 2002). For instance, immigration from localities where a given species experiences weaker interspecific than intraspecific competition (i.e. sources where the species can increase when rare) can prevent competitive exclusion in localities where it suffers stronger interspecific than intraspecific competition (i.e. sinks where the species cannot increase when rare). What is perhaps less well known is that this rescue effect imposes a cost of dispersal on sources. While emigration from source localities is necessary for maintaining species in sink localities, too much emigration can depress the per capita growth rates of sources and homogenize spatial variance in the strength of competition (Levin 1974; Pacala \& Roughgarden 1982; Amarasekare \& Nisbet 2001; Mouquet \& Loreau 2002). Such homogenization can cause region-wide exclusion of species that are inferior competitors or have lower initial abundances, thus reducing diversity below that observed in isolated communities.

The prediction that high dispersal rates should undermine diversity begs the question of whether there are biological mechanisms that can reduce the cost of dispersal to source communities. There are two basic mechanisms. The first involves strong spatial variation in the strength of competition; for example, if the competitive advantage in the source is strong relative to the competitive disadvantage in the sink, then the source may be able to withstand quite high rates of emigration. The second involves reducing the amount of emigration from source communities. One such mechanism is localized dispersal (e.g. Murrell \& Law 2003; Snyder \& Chesson 2003). If individuals do not move very far from their natal localities, they are less likely to leave areas favourable to their growth and reproduction. However, they are also less likely to rescue sink populations from extinction. Thus localized dispersal may increase source viability, but species diversity is likely to be lower than that observed under low levels of global dispersal. (Global here means a dispersal range that is sufficiently large to encompass both favourable and unfavourable localities of the landscape.) 
A second, and much less well-studied, mechanism for reducing emigration from sources is density-dependent dispersal. All models that predict diversity-reducing effects of high dispersal assume dispersal to be density independent and global in extent (e.g. Levin 1974; Pacala \& Roughgarden 1982; Shmida \& Ellner 1984; Amarasekare \& Nisbet 2001; Mouquet \& Loreau 2002). Models of localized dispersal also assume density independence (e.g. Murrell \& Law 2003; Snyder \& Chesson 2003). Most real organisms, however, exhibit some form of density-dependent dispersal (Sutherland et al. 2002). Investigation of global density-dependent dispersal is important because this type of dispersal has the potential to rescue sink populations from extinction without unduly affecting source viability. Thus, density-dependent dispersal could increase species diversity above that observed under densityindependent dispersal, whether local or global.

While several studies have investigated the role of density-dependent dispersal in the source-sink dynamics of single species (e.g. Pulliam 1988; Howe et al. 1991; Pulliam \& Danielson 1991; Amarasekare 2004), I am aware of only one study that has investigated density-dependent dispersal in the source-sink dynamics of competing species (Nishimura \& Kishida 2001). This study considered only one mode of dispersal (dispersal rate increasing in proportion to the species' density) for species engaged in dominance competition. Data, however, show that dispersal rate can both increase and decrease with increasing density (e.g. Denno \& Peterson 1995; Herzig 1995; Fonseca \& Hart 1996; Veit \& Lewis 1996; Doncaster et al. 1997; Wolff 1997; Diffendorfer 1998; Aars \& Ims 2000). Data also show that species engage in both dominance and pre-emptive competition, as well as combinations thereof. Therefore, a comprehensive investigation of how densitydependent dispersal influences source viability requires a comparative approach that considers different types of competition as well as different modes of dispersal.

Here, I present a model that investigates how spatial variation and density-dependent dispersal influence dispersal costs to sources under dominance and pre-emptive competition. I derive comparative predictions about how different types of competition, spatial variation and modes of dispersal influence source viability and hence sourcesink coexistence. I discuss the implications of these results for species coexistence in patchy environments.

\section{THE MODEL}

Consider a patchy environment consisting of multiple localities, each inhabited by a local community of competing species. Within a given locality, competition occurs according to Lotka-Volterra dynamics. The communities are linked by emigration and immigration of competing species. A collection of such communities that occupies a particular region of the landscape constitutes a metacommunity. Dispersal is global in that all species can get to all habitable localities within the metacommunity.

The dynamics of a source-sink system of two competing species inhabiting two localities ( $j$ and $l$ ) are given by

$$
\begin{aligned}
\frac{\mathrm{d} X_{1 j}}{\mathrm{~d} t}= & r_{1} X_{1 j}\left(1-\frac{X_{1 j}}{K_{1 j}}-\alpha_{1 j} \frac{X_{2 j}}{K_{1 j}}\right) \\
& -D_{1} X_{1 j}\left(\frac{X_{1 j}+\alpha_{1 j} X_{2 j}}{K_{1 j}}\right)^{s}+D_{1} X_{1 l}\left(\frac{X_{1 l}+\alpha_{1 l} X_{2 l}}{K_{1 l}}\right)^{s},
\end{aligned}
$$

$$
\begin{aligned}
\frac{\mathrm{d} X_{2 j}}{\mathrm{~d} t}= & r_{2} X_{2 j}\left(1-\frac{X_{2 j}}{K_{2 j}}-\alpha_{2 j} \frac{X_{1 j}}{K_{2 j}}\right) \\
& -D_{2} X_{2 j}\left(\frac{X_{2 j}+\alpha_{2 j} X_{1 j}}{K_{2 j}}\right)^{s}+D_{2} X_{2 l}\left(\frac{X_{2 l}+\alpha_{2 l} X_{1 l}}{K_{2 l}}\right)^{s},
\end{aligned}
$$

where $X_{i j}$ is the abundance, $\alpha_{i j}$ the competition coefficient and $K_{i j}$ the carrying capacity of species $i$ in locality $j(i, j$, $l=1,2 ; j \neq l$ ). The parameter $r_{i}$ is the intrinsic growth rate of species $i$, and $D_{i}$ is its per capita emigration rate. The quantity $s$ denotes the strength of density dependence in dispersal.

I non-dimensionalize equation (2.1) using scaled quantities. Non-dimensional analysis allows the dynamic system to be described by a minimal set of parameters and also illuminates the scaling relations between the various dynamic processes (Murray 1993).

I use the following substitutions:

$$
\begin{aligned}
& x_{i j}=\frac{X_{i j}}{K_{i j}}, a_{i j}=\alpha_{i j} \frac{K_{k j}}{K_{i j}}, k_{i}=\frac{K_{i l}}{K_{i j}}, \\
& \beta_{i}=\frac{D_{i}}{r_{i}}, \rho=\frac{r_{2}}{r_{1}}, \tau=r_{1} t(i, j, k, l=1,2 ; i \neq k, j \neq l),
\end{aligned}
$$

to transform equation (2.1) to its non-dimensional form.

The non-dimensional quantity $x_{i j}$ represents the density of species $i$ in locality $j$ scaled by its carrying capacity, and $a_{i j}$ represents the per capita competitive effect of species $m$ on species $i$ scaled by the ratio of their respective carrying capacities. The quantity $k_{i}$ is the ratio of the carrying capacities in the two localities for species $i$, and $\beta_{i}$ is its per capita emigration rate scaled by the intrinsic growth rate. The quantity $\rho$ is the ratio of the intrinsic growth rates of the two species. The non-dimensional time metric $\tau$ expresses time in terms of species 1's intrinsic growth rate.

I substitute the non-dimensional quantities into equation (2.1) to obtain the following system of equations.

$$
\begin{aligned}
\frac{\mathrm{d} x_{1 j}}{\mathrm{~d} \tau}= & x_{1 j}\left(1-x_{1 j}-a_{1 j} x_{2 j}\right)-\beta_{1} x_{1 j}\left(x_{1 j}+a_{1 j} x_{2 j}\right)^{s} \\
& +k_{1} \beta_{1} x_{1 l}\left(x_{1 l}+a_{1 l} x_{2 l}\right)^{s}, \\
\frac{\mathrm{d} x_{2 j}}{\mathrm{~d} \tau}= & \rho x_{2 j}\left(1-x_{2 j}-a_{2 j} x_{1 j}\right)-\rho \beta_{2} x_{2 j}\left(x_{2 j}+a_{2 j} x_{1 j}\right)^{s} \\
& +k_{2} \rho \beta_{2} x_{2 l}\left(x_{2 l}+a_{2 l} x_{1 l}\right)^{s} .
\end{aligned}
$$

Unless otherwise noted, all variables and parameters from this point on are expressed as scaled quantities.

The nature of density dependence in dispersal is mediated by the parameter $s$ (figure 1 ). When $s=0$, emigration is density independent and occurs at the per capita rate $\beta_{i}$. When $s \neq 0$, emigration is dependent on the effective density of the species (cf. Nishimura \& Kishida 2001), which is a function of both intraspecific and interspecific competition (e.g. $x_{i j}+a_{i j} x_{m j}, i, m=1,2 ; i \neq m$ ). There are two basic modes of density-dependent dispersal, depending on the sign of $s$. When $s<0$, emigration increases with density at a decelerating rate, akin to a Type II functional response at low resource abundances (Holling 1959). (The analogy is not perfect because the emigration rate does not necessarily saturate at high abundances; figure 1.) This mode of dispersal is likely to occur when individuals tend to emigrate from low-density populations, owing to difficulties in finding a mate or reduced 

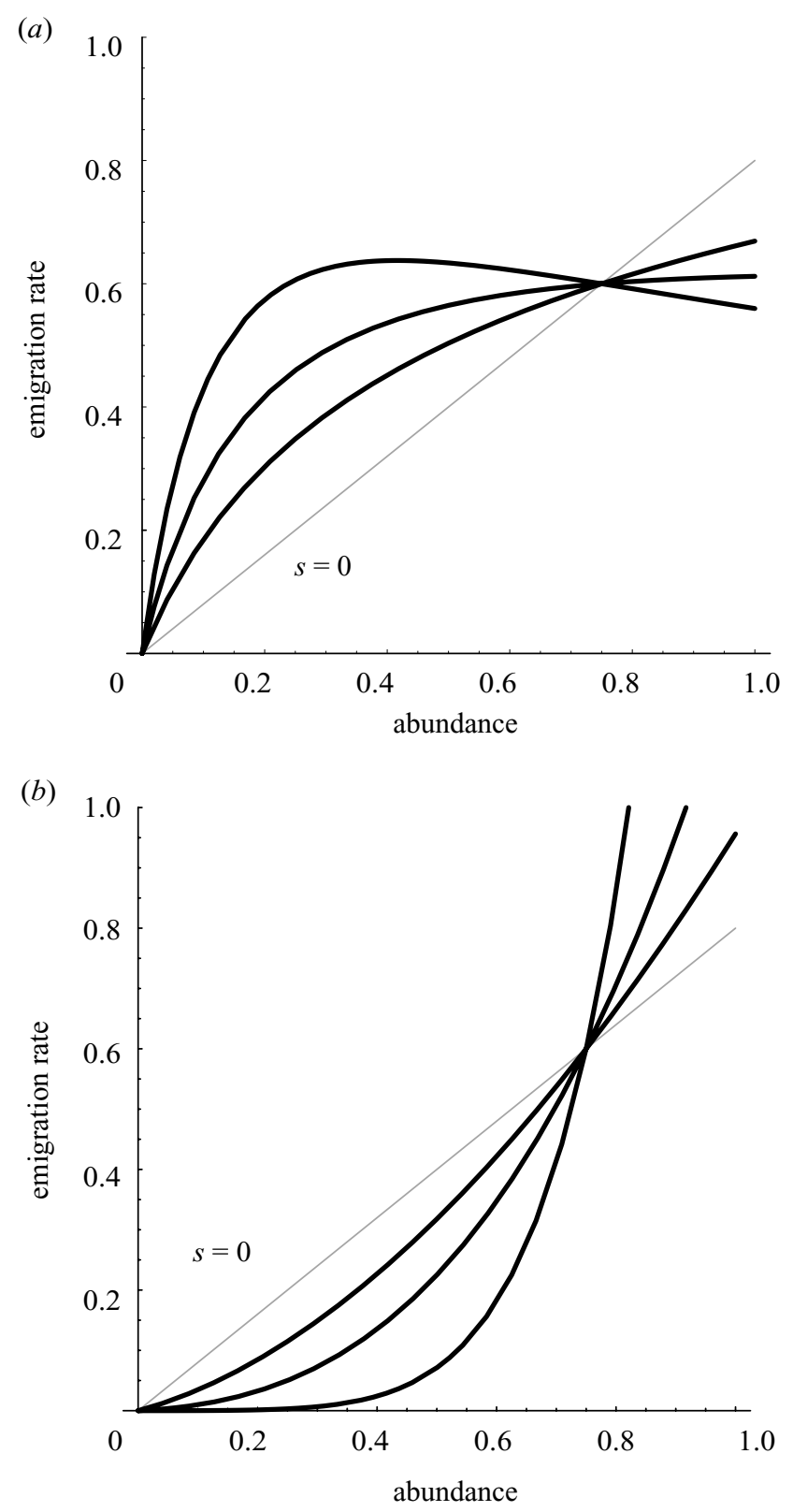

Figure 1. Modes of density-dependent dispersal. The $x$-axis is the abundance of species $i$ in locality $j\left(x_{i j}\right)$, and the $y$-axis is the emigration rate $\left(\beta_{i} x_{i j}\left(x_{i j}+\alpha_{i j} x_{k j}\right)^{s}\right)$. In the baseline case of density-independent dispersal $(s=0)$, emigration rate increases in proportion to density. (a) Type II densitydependent dispersal $(s<0)$ occurs when the emigration rate increases with abundance at a decelerating rate. (b) Type III density-dependent dispersal $(s>0)$ occurs when the emigration rate increases with abundance at an accelerating rate. Note that the dispersal function can be non-monotonic for $s \ll 0$, but this occurs for values of $s$ that are not biologically feasible.

predator vigilance. Examples include insects (Herzig 1995; Kuussaari et al. 1998), birds (Birkhead 1977) and mammals (Wolff 1997; Diffendorfer 1998). When $s>0$, emigration increases with density at an accelerating rate, akin to a Type III functional response at low resource abundances (Murdoch \& Oaten 1975). This mode of dispersal is likely to occur when individuals tend to leave high-density populations owing to strong intraspecific and interspecific competition. Examples include insects
(Denno \& Peterson 1995; Fonseca \& Hart 1996; Rhainds et al. 1998) and territorial birds and mammals (Veit \& Lewis 1996; Wolff 1997; Aars \& Ims 2000).

Although the dynamic analogy with functional responses is not perfect, it is convenient to refer to the three dispersal modes as Type I $(s=0)$, Type II $(s<0)$ and Type III $(s>0)$. Note that Type III densitydependent dispersal is a generalization of the mode of density-dependent dispersal considered in Pulliam's (1988) model of single-species source-sink dynamics. In Pulliam's model, intraspecific competition occurs for resources that are spatially well defined and easily defendable (e.g. nesting sites or breeding territories). Individuals obey a habitat-selection rule such that emigration from the source to the sink occurs only after all breeding sites in the source locality are filled. Thus, dispersal involves only 'surplus' individuals whose departure does not affect the source population's per capita growth rate. The functional form of Type III density-dependent dispersal that I employ here generalizes such surplus dispersal to resources that are not as well-defined or defendable (e.g. essential nutrients and mobile prey).

Because I am interested in spatial variation in the strength of competition and density-dependent dispersal, I assume that species differ in their per capita competitive effects $\left(\alpha_{i j}\right)$ and dispersal rates $\left(\beta_{i}\right)$ but are otherwise similar, i.e. $\rho=1, k_{1}=k_{2}, K_{1 j}=K_{2 j}(j=1,2)$. This yields the following simplified system:

$$
\begin{aligned}
\frac{\mathrm{d} x_{i j}}{\mathrm{~d} \tau}= & x_{i j}\left(1-x_{i j}-a_{i j} x_{m j}\right)-\beta_{i} x_{i j}\left(x_{i j}+a_{i j} x_{m j}\right)^{s} \\
& +\beta_{i} x_{i l}\left(x_{i l}+a_{i l} x_{m l}\right)^{s} \quad i, j, m, l=1,2 ; i \neq m, j \neq l .
\end{aligned}
$$

There are two basic types of competition to consider: dominance and pre-emption. This distinction is important because spatial variation arises in fundamentally different ways in the two types of competition, which in turn can cause significant differences in the dispersal costs incurred by source communities. Dominance competition implies that $\alpha_{1 j}<1$ and $\alpha_{2 j}>1(j=1,2)$, i.e. species 1 experiences greater intraspecific competition than interspecific competition, and species 2 experiences the opposite. Species 1 is hence the superior competitor and excludes species 2 in the absence of dispersal. Pre-emptive competition, on the other hand, implies that $\alpha_{1 j}>1$ and $\alpha_{2 j}$ $>1$, i.e. both species experience stronger interspecific competition than intraspecific competition. Neither species is hence the superior competitor, and the species with the higher initial abundance excludes the other in the absence of dispersal.

The key issue is whether spatial variation and densitydependent dispersal reduce dispersal costs to sources below those expected under density-independent dispersal. I investigate this issue using a comparative analysis of dominance and pre-emptive competition.

\section{(a) Dominance competition}

As noted above, under dominance competition species 1 is the superior competitor and excludes species 2 in the absence of dispersal. In a spatially heterogeneous environment, however, there may be biotic or abiotic factors that cause spatial variation in the strength of competition. For instance, the superior competitor may be more susceptible 
to a shared natural enemy whose abundance varies spatially, or to spatial variation in temperature, humidity or nutrient availability. Such variation allows the inferior competitor to increase when rare in localities where it suffers weaker interspecific than intraspecific competition (e.g. because high natural-enemy abundance causes high mortality in the superior competitor, or intolerance of a particular temperature-humidity regime reduces its resource-exploitation abilities) and to be driven extinct when rare in localities where it suffers stronger interspecific than intraspecific competition (e.g. because low natural-enemy abundances cause negligible mortality in the superior competitor, or temperature-humidity regimes enhance its resource-exploitation abilities). Spatial variation in the strength of competition thus creates a sourcesink structure, with favourable localities where species experience weak interspecific competition and unfavourable localities where they experience strong interspecific competition. Given such variation, dispersal from favourable to unfavourable localities can allow local coexistence by preventing competitive exclusion in the unfavourable localities. This leads to a spatial pattern with both species being present in all localities of the landscape (barring any dispersal constraints) as opposed to being restricted to the source localities in the absence of dispersal.

A prerequisite for local coexistence is mutual invasibility. Each species should be able to increase when rare when the other species is at its carrying capacity. Invasion is possible if the dominant eigenvalue of the Jacobian of equation (2.3) is positive when evaluated at the appropriate boundary equilibrium $\left(x_{11}^{*}, x_{12}^{*}, x_{21}^{*}, x_{22}^{*}\right)=(1,1,0,0)$ or $\left(x_{11}^{*}, x_{12}^{*}, x_{21}^{*}, x_{22}^{*}\right)=(0,0,1,1)$. A little algebra shows that invasion can occur if

$$
\begin{aligned}
& \left(1-\alpha_{i j}\right)\left(1-\alpha_{i l}\right)-\beta_{i}\left(\alpha_{i l}^{s}\left(1-\alpha_{i j}\right)\right. \\
& \left.\quad+\alpha_{i j}^{s}\left(1-\alpha_{i l}\right)\right)<0,
\end{aligned}
$$

where $1-\alpha_{i j}$ is the initial per capita growth rate of species $i$ in locality $j$ in the absence of dispersal $(i, j, l=1,2 ; j \neq l$ ).

The invasion criterion has two terms. The first term is the product of the initial growth rates of species $i$ in the two localities. The second term is the product of the per capita emigration rate and the weighted sum of the initial growth rates. Note that, when there is spatial variation in the strength of competition, one locality is a source and the other locality is a sink. Hence, the product of the initial growth rates is always negative. Invasibility therefore depends on the weighted sum of the initial growth rates. (In this model the coexistence equilibrium is stable when it is feasible, and hence invasibility also guarantees longterm coexistence.)

There are two situations under which invasion can occur. First, if the weighted sum of the initial growth rates is positive, invasion is possible as long as the emigration rate $\beta_{i}$ is non-zero. Because only one of the initial growth rates is positive, positivity of the weighted sum requires the competitive advantage that a given species experiences in the source to be very strong relative to the competitive disadvantage it suffers in the sink. The key point to appreciate is that when spatial variation in the strength of competition is very strong, there is no dispersal cost to the source. Local coexistence is possible regardless of how high the emigration rate is. By contrast, if the weighted sum of the initial growth rates is negative, invasion is possible only if the emigration rate is below a critical threshold:

$$
\beta_{i}<\frac{\left(1-\alpha_{i j}\right)\left(1-\alpha_{i l}\right)}{\left(\alpha_{i l}^{s}\left(1-\alpha_{i j}\right)+\alpha_{i j}^{s}\left(1-\alpha_{i l}\right)\right)} .
$$

Invasibility now depends on the interaction between spatial variation and dispersal, which in turn depends on the different forms of spatial variation and modes of dispersal.

As noted above, there are three basic modes of dispersal: Type I, Type II and Type III. Categorizing the different forms of spatial variation requires a consideration of the average competitive effect experienced by a given species across the landscape. When spatial variation is high, the competitive advantage in the source is stronger than the competitive disadvantage in the sink. As a result, intraspecific competition will be stronger than interspecific competition when averaged across source and sink localities. By contrast, when spatial variation is low, the competitive advantage in the source is weak compared with the competitive disadvantage in the sink. As a result, interspecific competition will be stronger than intraspecific competition when averaged across source and sink localities. Because each species can experience either of these two situations, the two-species system will exhibit three basic forms of spatial variation (Amarasekare \& Nisbet 2001).

The first case occurs when species 1 is the superior competitor in locality $1\left(\alpha_{11}<1\right.$ and $\left.\alpha_{21}>1\right)$ and species 2 is the superior competitor in locality $2\left(\alpha_{12}\right.$ $>1$ and $\alpha_{22}<1$ ) but the competitive advantage in the source is greater than the competitive disadvantage in the sink for both species. Hence the average competitive effect across the landscape is such that intraspecific competition is stronger than interspecific competition for both species $\left(\bar{\alpha}_{1}<1, \bar{\alpha}_{2}<1\right)$. I call this regional niche partitioning.

The second case occurs when species 1 is the superior competitor in locality 1 and species 2 is the superior competitor in locality 2 . Species 1 has a strong competitive advantage in the source, which overrides the disadvantage it suffers in the sink, but species 2 has only a weak advantage in the source, which is insufficient to overcome its disadvantage in the sink. Hence the average competitive effect across the landscape is such that species 1 is the superior competitor $\left(\bar{\alpha}_{1}<1, \bar{\alpha}_{2}>1\right)$. I call this regional dominance.

The third case occurs when species 1 is the superior competitor in locality 1 and species 2 is the superior competitor in locality 2 , but the competitive advantage in the source is weaker than the competitive disadvantage in the sink for both species. Hence the average competitive effect is such that interspecific competition is stronger than intraspecific competition for both species $\left(\bar{\alpha}_{1}>1, \bar{\alpha}_{2}\right.$ $>1$ ). I call this a regional priority effect.

Given that we have three forms of spatial variation and three modes of dispersal, the interplay between spatial variation and dispersal can lead to nine different outcomes. These are summarized in table 1 and figure 2 . There are two key points to note. First, invasibility depends strongly on the form of spatial variation. Invasion success is greatest under regional niche partitioning and least under a regional priority effect. This is because under regional niche partitioning the competitive advantage in the source is greater than the competitive disadvantage in 
Table 1. The interplay between spatial variation and the dispersal mode in determining local coexistence under dominance competition.

spatial variation

dispersal mode

regional niche partitioning

regional dominance ${ }^{\mathrm{b}}$

regional priority effect ${ }^{\mathrm{c}}$

\begin{tabular}{lccc}
\hline $\begin{array}{l}\text { Type I } \\
(s=0)\end{array}$ & $\beta_{i}>0$ & $\beta_{i}<\beta_{c}$ & $\beta_{i}<\beta_{c}$ \\
Type II & $\beta_{i}>0(s \rightarrow 0)$ & $\beta_{i}<\beta_{c}$ & $\beta_{i}<\beta_{c}$ \\
$(s<0)$ & $\beta_{i}<\beta_{c}(s \ll 0)$ & $\beta_{i}>0(s>1)$ & $\beta_{i}<\beta_{c}$ \\
Type III & $\beta_{i}>0$ & $\beta_{i}<\beta_{c}(0<s<1)$ &
\end{tabular}

a When dispersal is density independent $(s=0)$, the sum of the initial growth rates is always positive. Noting that $\alpha_{i j}<1, \alpha_{i l}>$ $1 \Rightarrow 1-\alpha_{i j}>0,1-\alpha_{i l}<0$, it can be seen that when dispersal is density dependent $(s \neq 0)$, the weighted sum of the initial growth rates will be positive only if $\left|\alpha_{i l}^{s}\left(1-\alpha_{i j}\right)\right|>\left|\alpha_{i j}^{s}\left(1-\alpha_{i l}\right)\right|, j, l=1,2 ; j \neq 1$. This condition is always satisfied under Type III density-dependent dispersal $(s>0)$. It can be satisfied under Type II density-dependent dispersal $(s<0)$ if $s \rightarrow 0$. When $s \ll 0$, the species that experiences the higher per capita competitive effect can invade only if its dispersal rate is below the threshold $\beta_{c}$ given by equation (2.5).

${ }^{b}$ When dispersal is density independent, the sum of the initial growth rates of the overall inferior competitor is always negative. Hence, invasibility requires that $\beta_{i}$ does not exceed the threshold $\beta_{c}$. With Type II density-dependent dispersal, the weighted sum is more negative than under density-independent dispersal, i.e. $\beta_{i}$ needs to be smaller than it does under density-independent dispersal. With Type III density-dependent dispersal, the weighted sum can be positive if $s \gg 1$. Thus, invasion is possible regardless of the magnitude of $\beta_{i}$ if Type III density-dependent dispersal is sufficiently strong.

${ }^{c}$ Because neither species is the superior competitor overall, the weighted sum of the initial growth rates cannot be positive for either species. Mutual invasibility requires the dispersal rates of both species to be below the threshold $\beta_{c}$.

(a)

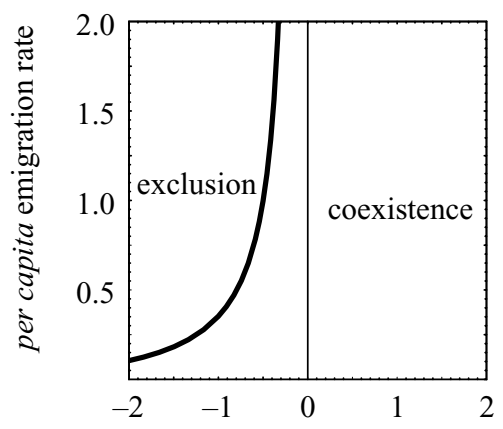

(b)

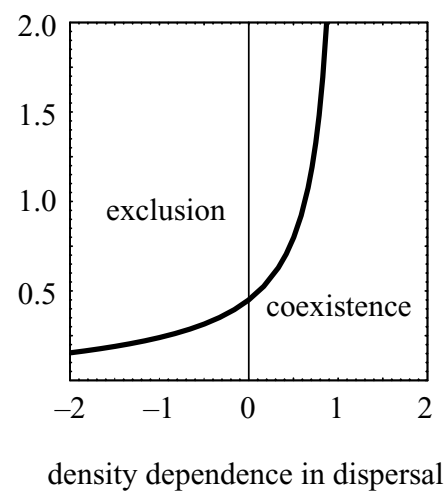

(c)

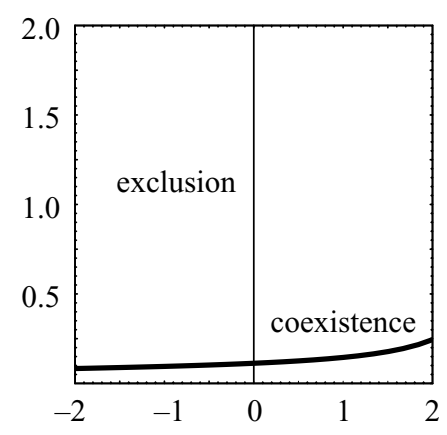

Figure 2. Effect of density-dependent dispersal on local coexistence under the three forms of spatial variation in the strength of dominance competition: (a) regional niche partitioning, (b) regional dominance and (c) regional priority effect. The $x$-axis denotes the strength of density dependence in dispersal $(s)$ and the $y$-axis denotes the per capita emigration rate of species 2 $\left(\beta_{2}\right)$, the overall inferior competitor. The solid curve depicts the dispersal threshold (equation (2.5)) that separates the region of the $s-\beta$ parameter space allowing local coexistence everywhere in the landscape from that which causes regional exclusion of species 2 . In general, Type III density-dependent dispersal $(s>0)$ facilitates coexistence, and Type II dispersal $(s<0)$ constrains it, compared with density-independent dispersal $(s=0)$. This effect, however, depends strongly on spatial variation in the strength of competition. Opportunities for coexistence are greatest under regional niche partitioning, and least under a regional priority effect. Parameter values are: $\alpha_{21}=1.5, \alpha_{22}=0.4$ for $(a), \alpha_{21}=1.9, \alpha_{22}=0.7$ for $(b)$ and $\alpha_{21}=1.9, \alpha_{22}=0.9$ for (c).

the sink for both species. It therefore requires quite a high rate of emigration to depress the per capita growth rate of the source community. Under a regional priority effect the competitive advantage in the source is weaker than the competitive disadvantage in the sink for both species. Hence even small amounts of emigration can depress the per capita growth rate of the source.

The second key point is that invasibility depends crucially on the mode of dispersal. Invasion success is greatest under Type III density-dependent dispersal and least under Type II density-dependent dispersal (table 1 and figure 2). This is because under Type III densitydependent dispersal the emigration rate is less than linear at low densities, so most individuals leave at high densities as a result of strong competition. Because these are surplus individuals that would not have contributed to local reproduction, this mode of dispersal incurs the least cost to source communities. Under Type II density-dependent dispersal, the emigration rate is disproportionately higher at lower abundances. This means that most individuals leave at low abundances, when competition is weak. Because these are individuals that could have contributed 
to local reproduction, this mode of dispersal can make the source's per capita growth rate negative at abundances well below the source carrying capacity. It thus incurs the greatest cost to source communities.

This interplay between spatial variance and densitydependent dispersal leads to two important insights. First, when spatial variation in the strength of competition is strong, source communities can withstand quite high rates of emigration. For instance, when spatial variation is such that regional niche partitioning results, local coexistence is possible with no dispersal cost to the source except under very strong Type II density-dependent dispersal (table 1; figure $2 a$ ). Second, even when spatial variation is not strong, density-dependent dispersal can reduce dispersal costs by preventing emigration from source communities. For instance, when Type III density-dependent dispersal is sufficiently strong, coexistence can occur with no dispersal costs to sources except when spatial variance is very low (table 1 ; figure 2 ).

The above results were obtained for dominance competition, where the outcome of competition depends on the species' competitive abilities. An important question is how dispersal costs to sources are affected when competition changes from dominance to pre-emption. I address this issue next.

\section{(b) Pre-emptive competition}

Under pre-emptive competition $\alpha_{1 j}>1, \alpha_{2 j}$ $>1(j=1,2)$, i.e. interspecific competition is stronger than intraspecific competition for both species. Hence, the species with the higher initial abundance excludes the other in the absence of dispersal. Levin (1974) has previously shown that local coexistence can occur with global density-independent dispersal, provided that different localities have different initial abundances of competing species. Dispersal incurs a cost to sources such that coexistence is possible only if the emigration rate is below a critical threshold. Too much emigration from the source to the sink homogenizes spatial variation in abundances, and the species with the higher overall abundance excludes the other (Levin 1974). The issue to investigate is what effects spatial variation and density-dependent dispersal have on coexistence.

Pure pre-emptive competition occurs when species have comparable competitive effects on each other (e.g. $\alpha_{1 j}=\alpha_{2 j}, j=1,2$ ) and differ only in their initial abundances. In this case invasibility is significantly more constrained than under dominance competition (figure $3 a, c$ ). This difference is a direct result of the way in which spatial variation arises under the two types of competition. Dominance competition involves spatial variation in species' competitive abilities, whereas pre-emptive competition involves spatial variation in species abundances. Thus even small amounts of emigration can disrupt the sourcesink structure by equalizing abundances across localities. Because spatial variation in the strength of pre-emptive competition is so easily destroyed, density-dependent dispersal, even when it is Type III, has only a marginal effect in reducing the costs of dispersal to sources.

Pure pre-emptive competition assumes that species are equivalent in their competitive effects. This is somewhat unrealistic given that most species in nature tend to show differences in competitive ability even when the outcome of competition is contingent on the initial conditions. Incorporation of such species differences leads to the notion of asymmetric pre-emptive competition, where species differ in the per capita competitive effects they experience (e.g. $\alpha_{1 j} \neq \alpha_{2 j}, j=1,2$ ). In the absence of dispersal, therefore, the species that suffers a lower per capita effect of interspecific competition has a larger domain of attraction for the boundary equilibrium with itself at carrying capacity and the other species extinct. This allows for spatial variation in the relative magnitudes of the competition coefficients (e.g. $\alpha_{1 j}, \alpha_{2 j}>1$ but $\bar{\alpha}_{1} \neq \bar{\alpha}_{2}$ ).

The key result is that, under asymmetric pre-emptive competition, invasion success becomes comparable to that under dominance competition (figure $3 a, b$ ). This is because spatial variation is much stronger when species differ in their per capita effects on each other. Thus it takes greater amounts of emigration to undermine the viability of source communities. This similarity in invasion success, however, belies the key differences between dominance and pre-emptive competition. Although spatial variation is key to reducing dispersal costs to sources and promoting local coexistence under both dominance and pre-emption, pre-emptive competition is much less sensitive to the dispersal mode than is dominance competition (figure 4). Under dominance competition the transition from Type I (density-independent) to Type II or Type III dispersal is associated with a large change in the parameter space that allows coexistence. Under pre-emptive competition, an equivalent transition induces only a marginal change in the parameter space that allows coexistence, particularly for Type II dispersal (figure 4). Thus, the diversityreducing effects of high dispersal persist under preemptive competition even when dispersal is density dependent, while they can be significantly mitigated under dominance competition if the density-dependent dispersal is Type III rather than Type II.

\section{DISCUSSION}

Competing species inhabiting patchy or fragmented environments can coexist if dispersal from favourable areas prevents competitive exclusion in unfavourable areas (e.g. Levin 1974; Pacala \& Roughgarden 1982; Shmida \& Ellner 1984; Amarasekare \& Nisbet 2001; Mouquet \& Loreau 2002). However, too much emigration from favourable (source) localities can undermine their viability and reduce diversity below that observed in isolated communities. Long-term coexistence, therefore, requires mechanisms that reduce the cost of dispersal to source communities.

Here, I investigate two biological mechanisms that reduce the cost of dispersal to source communities. The first involves increasing spatial variation in the strength of competition such that source communities can withstand high rates of emigration. The second involves reducing the amount of emigration from sources via density-dependent dispersal. A key finding is that, while both mechanisms are important in reducing dispersal costs under dominance competition, spatial variation is much more important than density-dependent dispersal under preemptive competition. For species engaged in dominance competition, spatial variation in the strength of competition is greatest under regional niche partitioning. In this 
(a)

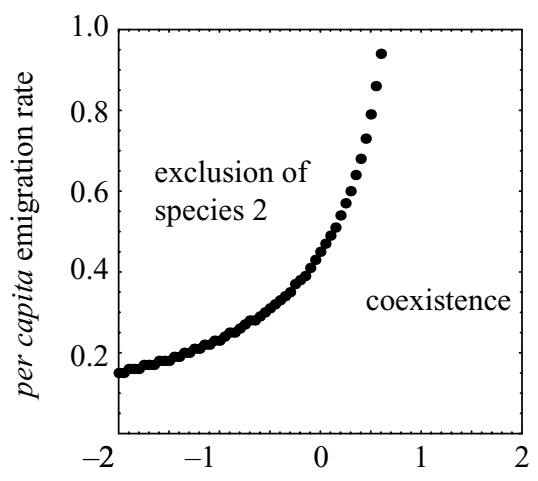

(b)

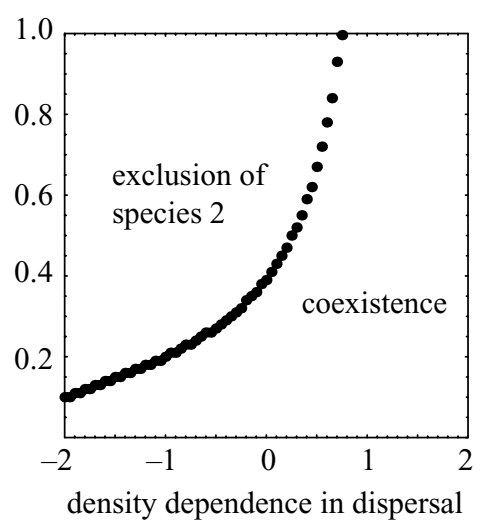

(c)

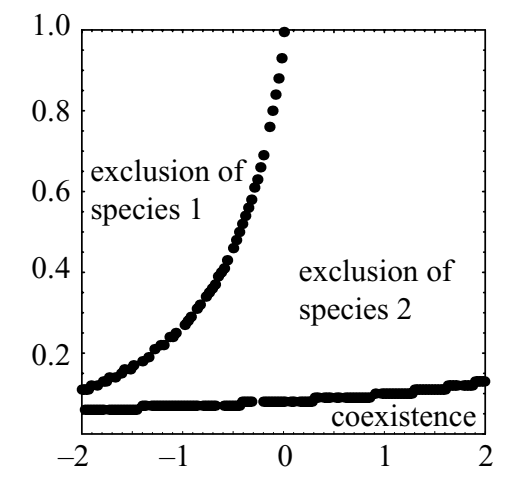

Figure 3. Effect of density-dependent dispersal on local coexistence under (a) regional dominance, $(b)$ asymmetric preemption and $(c)$ pure pre-emption. Under both regional dominance and asymmetric pre-emption, high rates of Type II (and to a lesser extent Type I) dispersal lead to the exclusion of species 2, the overall inferior competitor. Under pure pre-emption either species 1 or species 2 could be excluded depending on the relative magnitudes of $\beta_{1}$ and $\beta_{2}$. In general, coexistence is most restricted under pure pre-emptive competition, an effect that is not ameliorated even under strong Type III densitydependent dispersal. Parameter values are: $\alpha_{11}=0.2, \alpha_{12}=1.2, \alpha_{21}=1.9, \alpha_{22}=0.7$ for $(a), \alpha_{11}=1.4, \alpha_{12}=1.7, \alpha_{21}=2.4$, $\alpha_{22}=1.3$ for $(b), \alpha_{11}=\alpha_{12}=\alpha_{21}=\alpha_{22}=1.7$ for $(c)$, and $\beta_{1}=0.01$ for all three forms of competition.

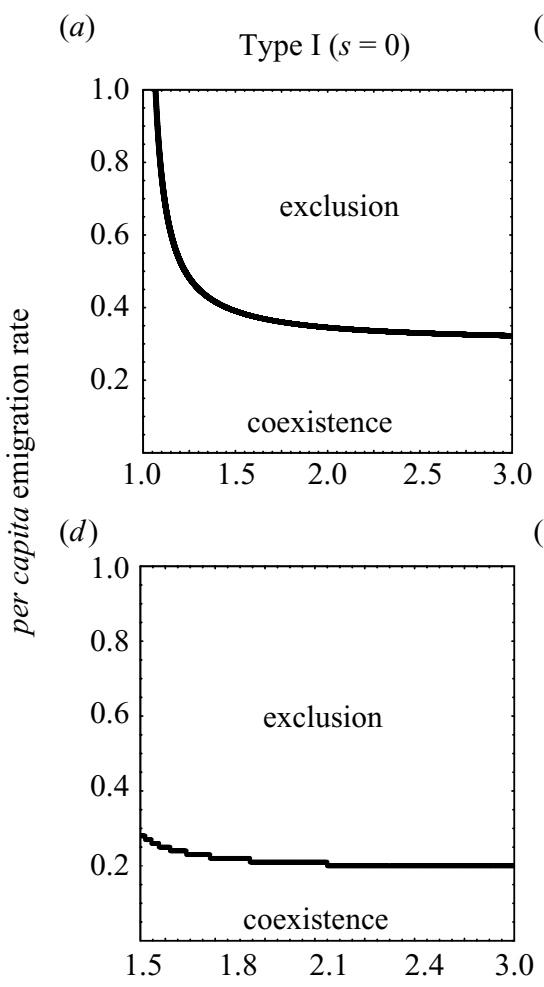

(b)

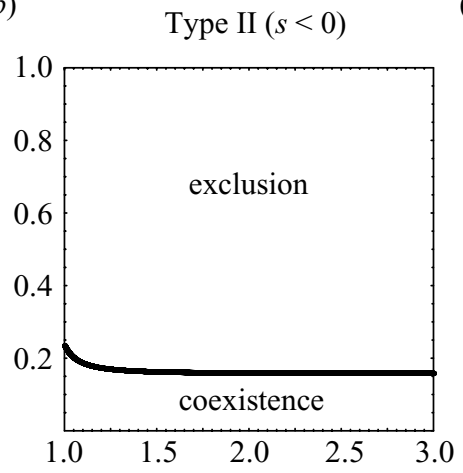

$(e)$

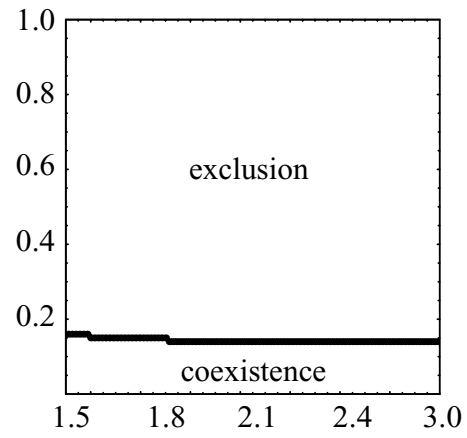

(c)

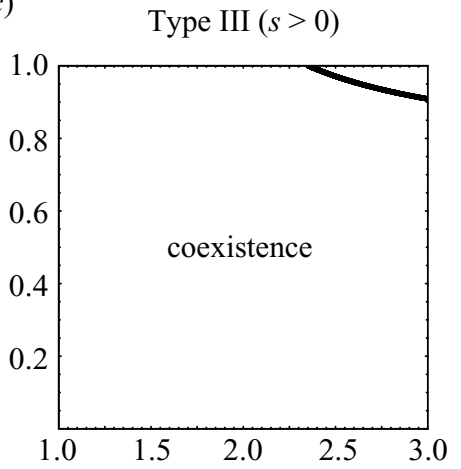

$(f)$

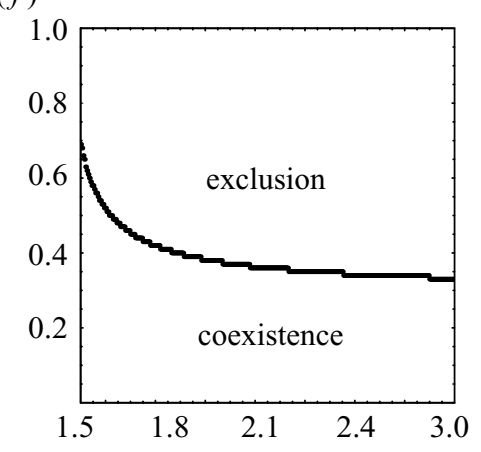

spatial variation in the strength of competition

Figure 4. Local coexistence in the face of spatial variation in the strength of competition for regional dominance $(a-c)$ and asymmetric pre-emptive competition $(d-f)$. Spatial variation is introduced by allowing $\alpha_{21}$ to vary (the $x$-axis) while holding all other competition coefficients constant. An increase in the magnitude of $\alpha_{21}$ implies a reduction in competitive ability and a reduction in spatial variation in the strength of competition for species 2 , the overall inferior competitor. The solid curve denotes the dispersal threshold that separates the parameter space allowing local coexistence from that which leads to competitive exclusion of species 2 . The threshold was calculated using equation (2.5) for dominance competition, and via numerical simulation for pre-emptive competition. Parameter values are: $\alpha_{22}=0.7$ for $(a-c), \alpha_{11}=1.1, \alpha_{12}=1.4, \alpha_{22}=1.1$, $\beta_{1}=0.01$ for $(d-f)$, and $s=-0.2$ and $s=1$, respectively, for Type II and Type III density-dependent dispersal.

case, coexistence is possible even under very high rates of emigration. By contrast, spatial variation is least under a regional priority effect. Now coexistence is possible only for very low emigration rates. For any given form of spatial variation under dominance competition, Type III densitydependent dispersal incurs the least cost to source communities. It thus increases local and regional diversity above that observed under density-independent dispersal. 
Table 2. Patterns of local and regional coexistence under different modes of density-independent and density-dependent dispersal. (Results apply to both dominance and pre-emptive competition, but the effects of dispersal mode are much weaker under preemptive competition.)

dispersal rate

dispersal mode

global, density independent

local, density independent

global, density dependent

Type II

global, density dependent

Type III

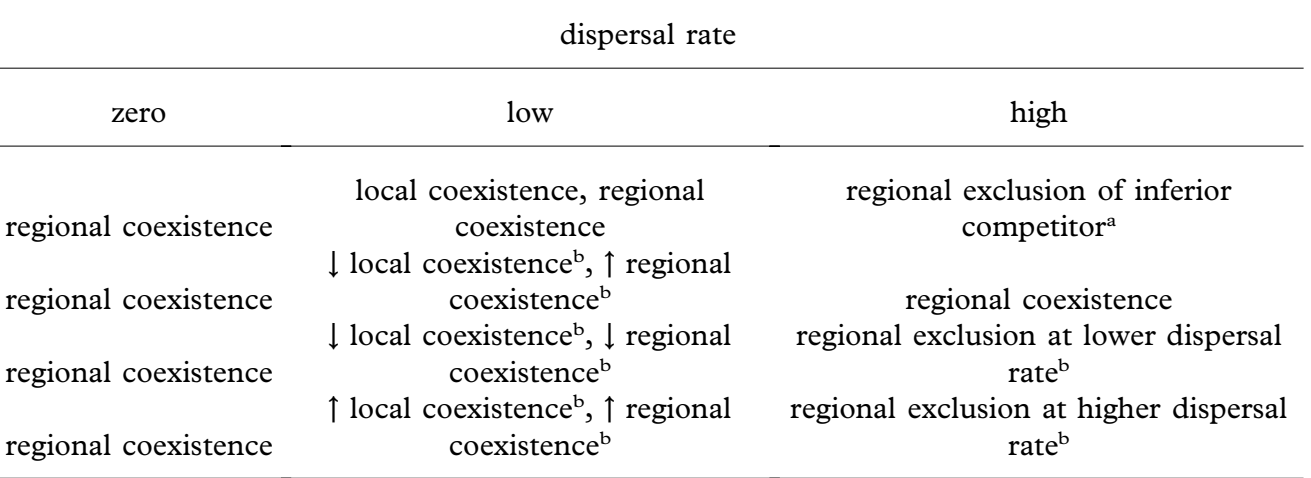

a The species with inferior competitive ability in dominance competition and lower initial abundance in pre-emptive competition.

${ }^{\mathrm{b}}$ Compared with global density-independent dispersal.

This is because most individuals leave at high densities owing to strong resource limitation. Because such surplus individuals play no role in local reproduction, emigration has no detrimental effect on the per capita growth rates of source communities. With Type III density-dependent dispersal, therefore, coexistence is possible even when spatial variation in the strength of competition is quite low. By contrast, Type II density-dependent dispersal incurs the greatest cost to source communities. It thus reduces local and regional diversity compared with densityindependent dispersal. This is because a disproportionately higher fraction of individuals leave at lower densities as a result of mate-finding difficulties or other social phenomena such as reduced predator vigilance. Thus emigration has a strong detrimental effect on the per capita growth rates of source communities. With this mode of dispersal coexistence is restricted even when spatial variation in the strength of competition is very high. In general, dispersal costs to sources are much greater, and hence local coexistence much more restrictive, under pre-emptive competition than under dominance competition. This is because spatial variation arises due to differences in initial abundances rather than due to differences in species' competitive abilities. Hence the balance between competition and dispersal is much more easily disrupted under pre-emptive competition. Introducing spatial variation in species' competitive abilities reduces dispersal costs by allowing sources to withstand higher rates of emigration, but density-dependent dispersal has only a marginal effect on dispersal costs.

These results provide for a comparative framework for the roles of competition and dispersal in local and regional coexistence (table 2). Previous studies have suggested that localized density-independent dispersal could prevent excessive emigration and allow initially rare species to build up densities in favourable localities (e.g. Murrell \& Law 2003; Snyder \& Chesson 2003). While localized density-independent dispersal does reduce dispersal costs to source communities, it also reduces the probability that sinks are rescued from extinction. Thus, localized densityindependent dispersal reduces local diversity compared with global density-independent dispersal. Global Type III density-dependent dispersal, however, can rescue sinks from extinction without undermining the viability of sources. One would therefore expect the greatest enhancement of local and regional diversity under this mode of dispersal. Type II density-dependent dispersal, because it has a stronger detrimental effect on source viability and a weaker rescue effect than density-independent dispersal, imposes the greatest constraints on local and regional diversity. In fact, the four modes of dispersal lead to four distinct patterns of local and regional coexistence (table 2). These predictions about diversity patterns, because they are comparative, have the advantage of allowing for simultaneous tests of multiple hypotheses about the interplay between competition and dispersal. This type of comparative analysis has not previously been conducted for competitive coexistence in the face of density-dependent dispersal. It is particularly informative because the competitive regimes considered include not only pure dominance and pure pre-emption but also intermediate situations, where spatial variation introduces an element of dominance into pre-emptive competition.

The predictions arising from the comparative analysis also identify taxa and communities that are likely to exhibit a particular pattern of diversity. For instance, species that emigrate at higher densities in response to strong competition (e.g. territorial invertebrates, birds and mammals; Birkhead 1977; Wolff 1997; Diffendorfer 1998; Sutherland et al. 2002) should experience an enhancement of local diversity in communities that are linked by dispersal compared with ones that are relatively isolated. By contrast, species that emigrate at lower densities in response to social phenomena such as mate-finding difficulties or reduced predator vigilance (e.g. plants, insects and mammals; Lamont et al. 1993; Herzig 1995; Wolff 1997; Diffendorfer 1998; Kuussaari et al. 1998) should experience a decrease in diversity in communities that are linked by dispersal compared with isolated ones. Similarly, species engaged in pure pre-emptive competition should be more susceptible to reductions in diversity as a result of perturbations that reduce spatial variation or increase emigration compared with species engaged in dominance competition or asymmetric pre-emption.

The above findings have important implications when applying source-sink theory to conservation problems. The scarcity of data, particularly for multi-species systems, necessitates making assumptions about density dependence 
in both population growth and dispersal. Knowing when density-independent dispersal provides a reasonable approximation to density-dependent dispersal is therefore crucial in implementing management strategies. In general, density-independent dispersal is not a reasonable approximation for species engaging in dominance competition that have a propensity to leave small populations owing to Allee effects, or large populations owing to strong resource limitation. In the former case, competing species are likely to exhibit Type II density-dependent dispersal, in which case density-independent dispersal overestimates the range of dispersal rates that allows local coexistence. In the latter case, competing species, particularly those that compete for spatially well-defined and defendable resources (e.g. nest sites or breeding territories), are likely to exhibit Type III density-dependent dispersal. In this case density-independent dispersal severely underestimates the range of dispersal rates that allows local coexistence. The same is true for species engaged in asymmetric pre-emptive competition. Thus, density-independent dispersal would yield an overly optimistic view of long-term coexistence when the actual dispersal mode is Type II, and an overly pessimistic view when the actual dispersal mode is Type III. It does, however, provide a reasonable approximation of both Type II and Type III densitydependent dispersal when species engaging in dominance competition exhibit a regional priority effect (figure 2) or when competition involves pure pre-emption (figure 3). Thus, density-independent dispersal can be used to predict long-term coexistence in communities where initial conditions or historical contingencies play a key role in competitive interactions (e.g. plants and sessile marine invertebrates), but not in communities structured by strong dominance hierarchies (e.g. insect parasitoid guilds, territorial invertebrates, birds and mammals).

The model presented here considers a two-species two-locality system, which was done in the interests of analytical tractability. The model can easily be extended to multiple interacting species inhabiting multiple localities. An important future direction would be to use a multi-species model to investigate community properties such as species richness and biomass under different regimes of competition and dispersal. Such an analysis can yield expectations about community patterns that can be contrasted with predictions from the neutral theory of biodiversity (Hubbell 2001) and niche-based but variationindependent mechanisms such as competition-colonization trade-offs. A second future direction involves investigating the role of dispersal strategies in competitive coexistence. Most real organisms exhibit age- or sex-biased dispersal strategies, but such variability is typically ignored in dynamic models of species coexistence. Investigations of how such strategies influence spatial coexistence allow for the development of a conceptual framework that integrates individual-level behavioural phenomena with the community-level dynamic processes that determine diversity patterns.

This research was supported by NSF grant DEB-0129270 and the Louise R. Block Fund from the University of Chicago. Two anonymous referees provided many helpful comments on the manuscript.

\section{REFERENCES}

Aars, J. \& Ims, R. A. 2000 Population dynamic and genetic consequences of spatial density-dependent dispersal in patchy populations. Am. Nat. 155, 252-265.

Amarasekare, P. 2004 The role of density-dependent dispersal in single species source-sink dynamics. F. Theor. Biol. 226, $159-168$

Amarasekare, P. \& Nisbet, R. 2001 Spatial heterogeneity, source-sink dynamics and the local coexistence of competing species. Am. Nat. 158, 572-584.

Birkhead, T. R. 1977 The effect of habitat and density on breeding success of the common guillemot (Uria aalge). $\mathcal{F}$. Anim. Ecol. 46, 751-764.

Codeco, C. T. \& Grover, J. P. 2001 Competition along a spatial gradient of resource supply: a microbial experimental model. Am. Nat. 157, 300-315.

Denno, R. F. \& Peterson, M. A. 1995 Density-dependent dispersal and its consequences for population dynamics. In Population dynamics (ed. N. Cappuccino \& P. W. Price), pp. 113-130. San Diego, CA: Academic.

Diffendorfer, J. E. 1998 Testing models of source-sink dynamics and balanced dispersal. Oikos 81, 417-433.

Doncaster, C. P., Colbert, J., Doligez, B., Gustafsson, L. \& Danchin, E. 1997 Balanced dispersal between spatially varying local populations: an alternative to the source-sink model. Am. Nat. 150, 424-445.

Fonseca, D. M. \& Hart, D. D. 1996 Density-dependent dispersal of black fly neonates is mediated by flow. Oikos $\mathbf{7 5}$, $49-58$.

Herzig, A. L. 1995 Effects of population density on long-distance dispersal in the goldenrod beetle Trirhabda virgata. Ecology 76, 2044-2054.

Holling, C. S. 1959 Some characteristics of simple types of predation and parasitism. Can. Entomol. 91, 385-398.

Howe, R. W., Davis, G. J. \& Mosca, V. 1991 The demographic significance of sink populations. Biol. Conserv. 57, 239-255.

Hubbell, S. P. 2001 The unified neutral theory of biodiversity and biogeography: monographs in population biology. Princeton University Press.

Kishimoto, K. 1990 Coexistence of any number of species in the Lotka-Volterra competitive system over two patches. Theor. Popul. Biol. 38, 149-158.

Kuussaari, M., Sacceri, I., Camara, M. \& Hanski, I. 1998 Allee effect and population dynamics in the Glanville fritillary butterfly. Oikos 82, 384-392.

Lamont, B. B., Klinkhamer, P. G. L. \& Witkowski, E. T. F. 1993 Population fragmentation may reduce fertility to zero in Banksia goodii: a demonstration of the Allee effect. Oecologia 94, 446-450.

Levin, S. A. 1974 Dispersion and population interactions. Am. Nat. 108, 207-228.

Mouquet, N. \& Loreau, M. 2002 Coexistence in metacommunities: the regional similarity hypothesis. Am. Nat. 159, $420-426$.

Murdoch, W. W. \& Oaten, A. 1975 Predation and population stability. Adv. Ecol. Res. 9, 1-131.

Murray, J. D. 1993 Mathematical biology. New York: Springer.

Murrell, D. J. \& Law, R. 2003 Heteromyopia and the spatial coexistence of similar competitors. Ecol. Lett. 6, 48-59.

Nishimura, K. \& Kishida, O. 2001 Coupling of two competitive systems via density-dependent migration. Ecol. Res. 16, 359-368.

Pacala, S. W. \& Roughgarden, J. 1982 Spatial heterogeneity and interspecific competition. Theor. Popul. Biol. 21, 92-113.

Pulliam, H. R. 1988 Sources, sinks and population regulation. Am. Nat. 132, 652-661. 
Pulliam, H. R. \& Danielson, B. J. 1991 Sources, sinks and habitat selection: a landscape perspective on population dynamics. Am. Nat. 137, 50-66.

Rhainds, M., Gries, G. \& Saleh, A. 1998 Density and pupation site of apterous female bagworms, Metisa plana (Walker) (Lepidoptera: Psychidae), influence the distribution of emergent larvae. Can. Entomol. 130, 603-613.

Shmida, A. \& Ellner, S. 1984 Coexistence of plant species with similar niches. Vegetation 58, 29-55.

Snyder, R. \& Chesson, P. 2003 Local dispersal can facilitate coexistence in the presence of permanent spatial heterogeneity. Ecol. Lett. 6, 301-309.
Sutherland, L. J., Gill, J. \& Norris, K. 2002 Density-dependent dispersal in animals: concepts, evidence, mechanisms and consequences. In Dispersal ecology (ed. J. Bullock), pp. 134151. Cambridge University Press.

Veit, R. \& Lewis, M. A. 1996 Dispersal, population growth, and the Allee effect: dynamics of the house finch invasion of eastern North America. Am. Nat. 148, 255-274.

Wolff, J. O. 1997 Population regulation in mammals: an evolutionary perspective. F. Anim. Ecol. 66, 1-13.

As this paper exceeds the maximum length normally permitted, the author has agreed to contribute to production costs. 


\author{
PRIYANGA AMARASEKARE \\ Department of Ecology and Evolution, University of Chicago, 1101 East 57th Street, Chicago, IL 60637, USA
}

\begin{abstract}
Summary
1. I present a model of mutualistic interactions in a patchy landscape. The interaction is between two species that differ in their mobility. The landscape is spatially structured, with several localities interconnected by dispersal of the mobile mutualist.

2. Within a given locality, an Allee effect can occur such that the per capita growth rate of the non-mobile mutualist declines with its own abundance. The Allee effect arises in response to low abundances of the mobile mutualist, and causes extinction of both species once their numbers fall below a critical threshold.
\end{abstract}

3. Dispersal of the mobile mutualist can rescue such sink communities from extinction, provided there is at least one source community in which both species have abundances above the extinction threshold. Dispersal itself is density-independent, but induces negative density-dependence that counteracts the positive density-dependence due to the Allee effect. This negative feedback effect of dispersal has not previously been demonstrated in mutualism models.

4. Rescue of sink communities, however, depends on how dispersal influences the local dynamics of source communities. If dispersal involves surplus individuals the loss of whom does not affect the reproductive output of the source community, persistence of sink communities is guaranteed as long as the survivorship of long-distance dispersers exceeds a lower threshold. In contrast, if dispersal involves emigrants that constitute a fraction of the source community's reproductive output, persistence of sink communities additionally requires that emigration does not exceed an upper threshold. Too much emigration can cause a net loss of the mobile mutualist from the source community, resulting in landscape-wide extinction of the mutualistic interaction.

5. The effect of dispersal mode on the dynamics of mutualistic interactions has not been appreciated previously. If the benefit of the rescue effect to sinks is outweighed by the cost of dispersal to sources, mutualistic communities linked by dispersal may experience a greater loss of diversity than communities that are isolated.

Key-words: Allee effect, coexistence, emigration, immigration, metacommunity, mutualism, spatial heterogeneity, source-sink dynamics.

Journal of Animal Ecology (2004) 73, 128-142

\section{Introduction}

Mutualisms represent an important class of species interactions. They occur in a wide variety of taxa from viruses to mammals, and range from highly specialized obligate mutualisms to generalized facultative interactions.

Empirical studies of mutualistic interactions suggest that habitat fragmentation can lead to an Allee effect, a reduction in the per capita growth rate with declining abundances (Allee 1931). For example, plant species relying on animal vectors for pollination and seed dispersal suffer a reduction in reproductive success
Correspondence: P. Amarasekare. Tel: +1 7738347647. Fax: +1 773702 9740. E-mail: amarasek@uchicago.edu. at low abundances because small patch sizes and increased isolation limit pollen transfer and prevent seed dispersal (Jennersten 1988; Lamont, Klinkhamer \& Witkowski 1993; Aizen \& Feinsinger 1994; Agren 1996; Kunin \& Iwasa 1996; Groom 1998). Concern has also been mounting over region-wide declines of insect pollinators (Cane 2001; Packer \& Owen 2001). Such declines are likely to intensify the Allee effects experienced by plant species in fragmented habitats.

Empirical evidence of Allee effects in fragmented landscapes underscores the importance of dispersal for the long-term persistence of mutualistic interactions. Although spatial theory for Allee effects in single species is relatively well developed (Dennis 1989; Lewis \& Kareiva 1993; Amarasekare 1998; Gyllenberg, Hemminki 

of mutualisms
(C) 2004 British

Ecological Society, Journal of Animal Ecology, 73 , $128-142$
\& Tammaru 1999; Keitt, Lewis \& Holt 2001), spatial theory for Allee effects that arise due to species interactions is not. In fact, there is very little theory on the spatial dynamics of mutualistic interactions (Hutson, Law \& Lewis 1985; Armstrong 1987). The lack of a predictive theoretical framework has in turn hampered empirical progress on dispersal effects on mutualisms.

Here I present a metacommunity model of mutualistic interactions that incorporates hierarchical spatial structure. The model yields comparative predictions about how population structure and modes of dispersal influence the persistence of obligate vs. facultative mutualisms. It also lends itself to investigations of how habitat destruction affects mutualistic interactions in a patchy environment.

\section{The model}

Consider a mutualistic interaction between two species that differ in their mobility. The obvious examples are plants and pollinators or plants and seed dispersers. Less obvious examples include coviruses of plants, RNA viruses in which the genome is split into two particles that code for replication and transmission; although the particle carrying the gene for replication can multiply within a plant, it cannot be transmitted from plant to plant without the presence of the particle that codes for encapsulation (Peden \& Symons 1976; Frankel-Conrat \& Wagner 1977).

The mutualists inhabit a spatially structured landscape consisting of a set of localities. Each locality consists of a large number of identical patches. A given patch is either empty or occupied by one or both species. A locality thus constitutes a local community of mutualists. A collection of localities linked by dispersal of the mobile mutualist constitutes a metacommunity.

\section{MUTUALISTIC DYNAMICS WITHIN AN ISOLATED LOCALITY}

The point of departure is a patch occupancy model developed by Armstrong (1987). For convenience, I will refer to the interacting species as plant and pollinator.

The dynamics of the mutualistic interaction within a single locality are given by:

$d p_{1} / d t=c_{1} p_{2}\left(h-p_{1}-p_{2}\right)-c_{2} p_{1} p_{2}-e_{1} p_{1}$

$d p_{2} / d t=c_{2} p_{1} p_{2}-e_{2} p_{2}$

eqn 1

where $p_{1}$ is the fraction of patches occupied by the plant and $p_{2}$, the fraction of patches occupied by both plant and pollinator. The parameter $h$ is the total amount of suitable habitat within the locality $(0<h \leq 1)$. The interaction is obligate in that the pollinator cannot survive in the absence of the plant, while the latter is able to survive in the absence of the former but cannot reproduce without it. Because the pollinator does not occur by itself, the fraction of empty patches is $h-p_{1}-$ $p_{2}$. The parameters $c_{1}$ and $c_{2}$ are, respectively, the per capita rates at which plant propagules colonize empty patches and pollinator propagules colonize plant patches. They encapsulate both local reproduction and subsequent establishment of offspring via random dispersal. Thus, seeds set by adult plants in plantpollinator patches colonize and establish in empty patches at a rate $c_{1} p_{2}\left(h-p_{1}-p_{2}\right)$, while pollinator propagules produced in plant-pollinator patches colonize and establish in plant patches at a rate $c_{2} p_{1} p_{2}$. Plant patches become extinct at a per capita rate $e_{1}$, and plant-pollinator patches at a rate $e_{2}$. These extinction rates encapsulate all forms of density-independent mortality experienced by adult plants and pollinators. It is assumed that extinction of plants from plant-pollinator patches causes immediate extinction of the pollinator (Armstrong 1987). Independent extinctions of plant and pollinator from patches occupied by both species do not alter the qualitative outcome of this model or those that follow.

Equation 1 yields three feasible equilibria: the trivial equilibrium $\left(p_{1}^{*}, p_{2}^{*}\right)=(0,0)$ and two interior equilibria $\left(p_{1}^{*}, p_{2}^{*}\right)=\left(\frac{e_{2}}{c_{2}}, \frac{c_{1}\left(c_{2} h-e_{2}\right)-c_{2} e_{2} \pm \sqrt{\left(c_{1}\left(c_{2} h-e_{2}\right)-c_{2} e_{2}\right)^{2}-4 c_{1} c_{2} e_{1} e_{2}}}{2 c_{1} c_{2}}\right)$. The trivial equilibrium and the larger of the interior equilibria are simultaneously stable. The smaller of the interior equilibria is a saddle.

The key results of this model are as follows: stable coexistence is possible as long as the pollinator colonization rate $c_{2}$ is not too low, and abundances of both plant and pollinator exceed a critical threshold (Fig. 1a,b). If abundances fall below this threshold, an Allee effect occurs and both plant and pollinator go extinct.

The positive density-dependence underlying the Allee effect arises in response to the abundance of the mutualistic partner. When the abundance of plantpollinator patches is high, propagule production by plants is also high. Because a large number of propagules are competing for a small number of empty patches, the per capita growth rate of plant patches declines as its own abundance increases. This creates negative densitydependence of the per capita growth rate. However, as the abundance of plant-pollinator patches falls, propagule production by plants also declines. As only a small number of propagules are available to colonize the increasingly large number of empty patches, the per capita growth rate of the plant patches starts to decline as its own abundance decreases. This leads to positive density-dependence in the per capita growth rate. It is the differential response of the plant per capita growth rate to pollinator abundance that leads to the hyperbolic shape of the isocline for plant patches (Fig. 1).

The general implication is that in an obligate mutualism involving two species that differ in their mobility, the mobile species may be unable to invade when rare even when it has a high rate of local reproduction and establishment. The ability to invade when rare is crucial, both in maintaining mutualistic interactions in already established areas and in spreading to new habitats. The key issue therefore is to determine whether there are 
130

P. Amarasekare

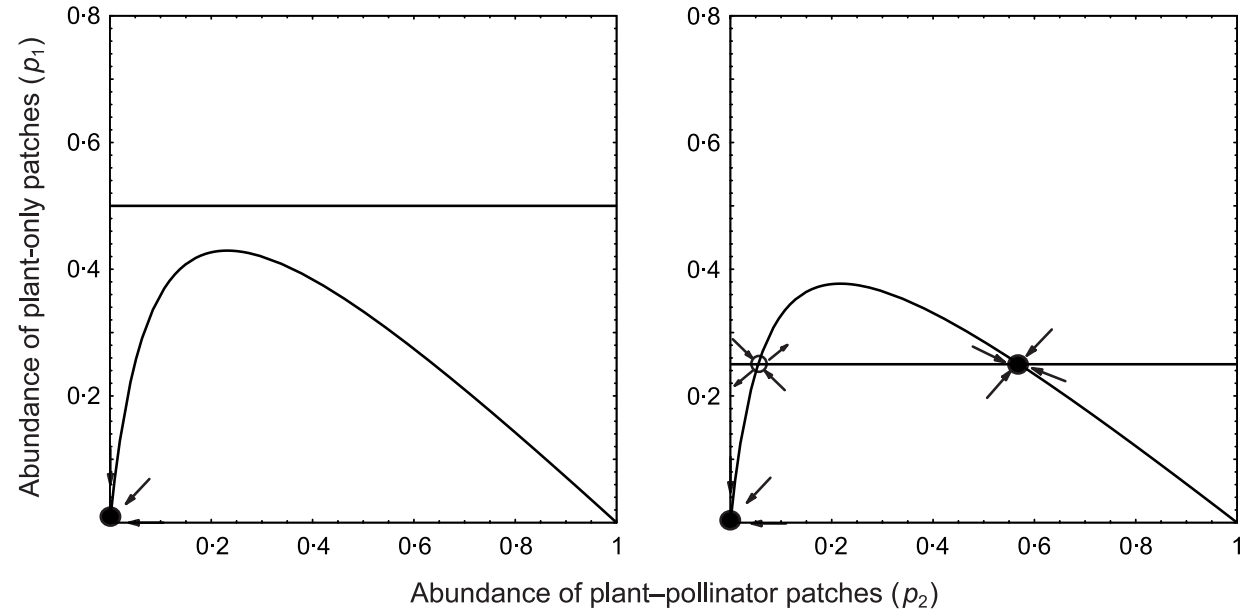

Fig. 1. Phase plots for mutualistic dynamics within an isolated locality (equation 1). The hyperbola is the zero isocline for the plant patches and the horizontal line, the zero isocline for the plant-pollinator patches. The black dots represent attractors and the open circle, a saddle point. When pollinator colonization rate is too low $\left(c_{2}=1\right)$, neither species can persist (left panel). For sufficiently high pollinator colonization rates $\left(c_{2}=3\right)$, coexistence of plant and pollinator is possible provided the abundance of both exceed the threshold defined by the saddle point. Note that an increase in the pollinator colonization rate reduces the height of the hump of the plant isocline (compare left and right panels). This occurs because higher pollinator colonization rates reduce the strength of the Allee effect, i.e. they reduce the abundance of the plant patches required to shift the nature of density-dependence from positive to negative. Other parameter values are: $c_{1}=5, e_{1}=e_{2}=0 \cdot 5, h=1$. conditions that allow the mobile species to invade when rare. Spatial dynamics provide a potential avenue for ameliorating Allee effects and allowing invasibility (Hutson et al. 1985; Amarasekare 1998; Gyllenberg et al. 1999). I investigate this problem by extending Armstrong's model to incorporate hierarchical spatial structure and dispersal between localities.

Although obligate mutualisms are less common in nature than facultative ones (Hoeksema \& Bruna 2000), they represent the worst case scenario in terms of susceptibility to Allee effects (May 1978; Vandermeer \& Boucher 1978; Dean 1983; Wells 1983; Wolin 1985; Anstett, Hossaert-McKey \& McKey 1997) and habitat destruction (Bond 1994; Anstett et al. 1997). Hence, obligate mutualisms provide a logical starting point for investigating the role of spatial dynamics. I later extend the model to consider facultative mutualisms.

\section{MUTUALISTIC DYNAMICS WITHIN A METACOMMUNITY}

Consider a region of the landscape that contains several localities. Dynamics within each locality occur as above (equation 1). The localities are now linked by dispersal of the mobile mutualist (e.g. pollinator).

The dynamics are given by the following general model:

$\frac{d p_{1 j}}{d t}=f_{j}\left(h_{j}, p_{i j}\right)-g_{j}\left(p_{i j}, a_{2 j}\right)-e_{1 j}\left(p_{1 j}\right)$

(C) 2004 British

Ecological Society, Journal of Animal Ecology, 73,

$128-142$ $\frac{d p_{2 j}}{d t}=g_{j}\left(p_{i j}, a_{2 j}\right)-e_{2 j}\left(p_{2 j}\right)$ tion of plant-pollinator patches in locality $j(i=1,2, j$ $=1, \ldots, n)$. The latter now depends on two additional quantities: the abundance of pollinators in locality $k$ $(k \neq j)$ and the parameter $a_{2 j}$, which describes pollinator dispersal between localities. The functions $e_{i j}(i=1,2)$ represent loss rates of plant and plant-pollinator patches, and $h_{j}$ is the fraction of suitable habitat in locality $j$.

Within an isolated locality, an Allee effect can cause the extinction of the mutualistic interaction. Can hierarchical spatial structure and dispersal between localities mitigate or eliminate the Allee effect?

\section{Single locality connected to a mainland source}

The simplest instance of hierarchical spatial structure is a single locality that receives colonists from a mainland source. The dynamics of such a locality are given by:

$\frac{d p_{1 L}}{d t}=c_{1 L} p_{2 L}\left(h_{L}-p_{1 L}-p_{2 L}\right)-c_{2 L} p_{1 L}\left(p_{2 L}+I p_{2 M}\right)-e_{1 L} p_{1 L}$

$\frac{d p_{2 L}}{d t}=c_{2 L} p_{1 L}\left(p_{2 L}+I p_{2 M}\right)-e_{2 L} p_{2 L}$

eqn 3

where $p_{1 L}$ and $p_{2 L}$ denote the fraction of plant and plant-pollinator patches within the focal locality, and $p_{2 M}$ is the abundance of plant-pollinator patches in the mainland. The mainland is assumed to be unaffected by its interaction with the locality, so $p_{2 M}$ is a constant. eqn 2 The total amount of suitable habitat is also assumed to be constant, i.e. $h_{L}+h_{M}=1\left(h_{L}<h_{M}\right)$. The dispersal parameter $I$ is the fraction of emigrants from the mainland that colonize plant patches in the focal locality; it can also be interpreted as the ratio of colonization rates of mainland vs. local colonists. (The distinction where the function $f_{j}$ describes the rate of production of plant patches, and the function $g_{j}$, the rate of produc- 
131

Spatial dynamics of mutualisms

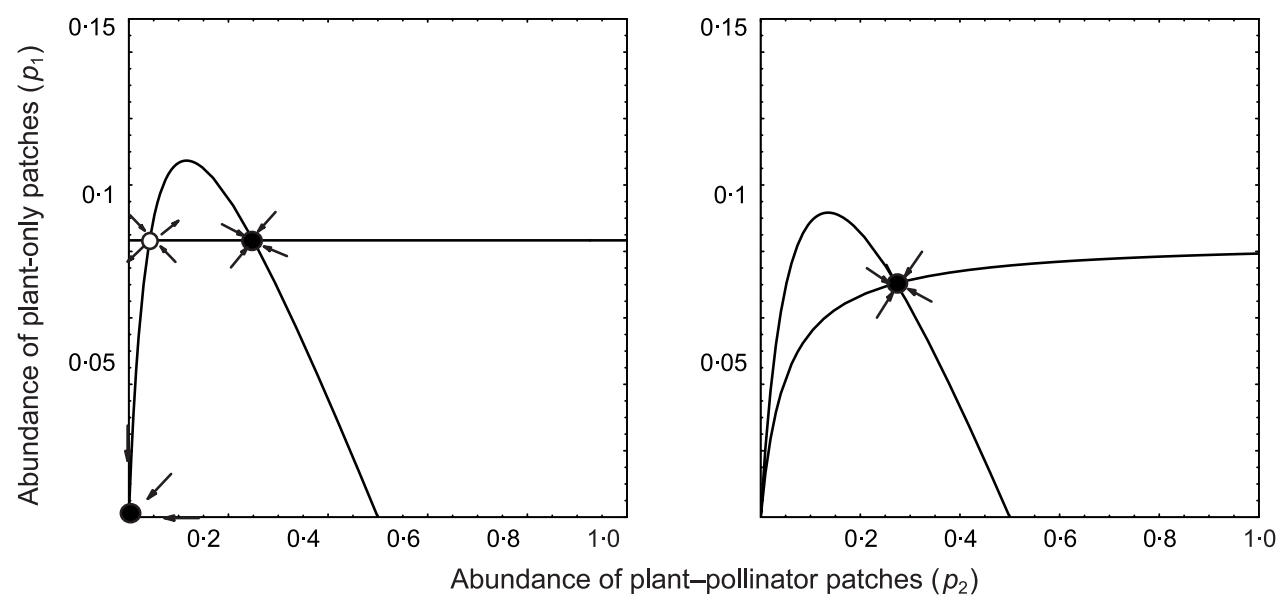

Fig. 2. Phase plots when a single locality is connected to a mainland source (equation 3 ). The plot on the left is for an isolated locality $(I=0)$, and the plot on the right for a locality that receives colonists from the mainland $(I>0)$. An input of colonists from the mainland removes the Allee effect and allows for stable coexistence of plant and pollinator. Parameter values are: $c_{1 L}=4$, $c_{2 L}=6, e_{1 L}=e_{2 L}=0 \cdot 5, h_{L}=0 \cdot 5$.

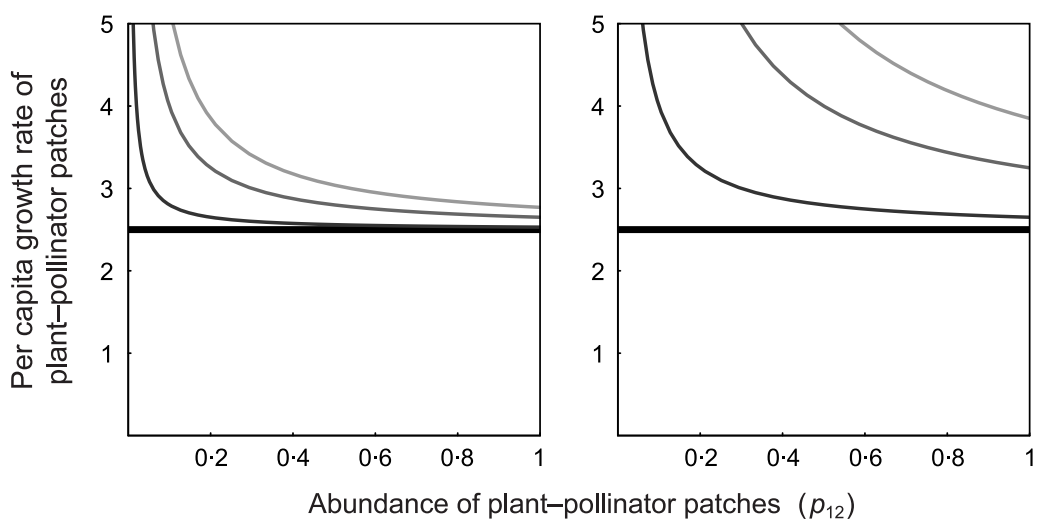

Fig. 3. Per capita growth rate of the plant-pollinator patches as a function of their abundance when a single locality is connected to a mainland source (equation 4). The left panel is for low pollinator dispersal $(I=0 \cdot 1)$, and the right panel, for high dispersal $(I=0 \cdot 5)$. In each panel, the horizontal line is the per capita growth rate in an isolated locality. The curves depict the per capita growth rate when the locality is linked to a mainland source, with decreasing concavity corresponding to increasing abundance of plant-pollinator patches in the mainland $\left(p_{2 M}=0 \cdot 1,0 \cdot 5,0 \cdot 9\right)$. An input of colonists results in an augmentation of the per capita growth rate, the effect of which is greater for higher levels of dispersal and higher abundances of plant-pollinator patches in the mainland. Parameter values are: $c_{2 L}=6, e_{2 L}=0 \cdot 5, h_{L}=0.5$.

between the two will become clearer when we consider the full model below.) The key result of this model is as follows: when the focal locality is connected to a mainland source, the Allee effect disappears (Fig. 2). There is a single, globally stable equilibrium corresponding to coexistence of plant and pollinator. Coexistence is guaranteed as long as there is an input of colonists from the mainland.

What causes the disappearance of the Allee effect? The per capita growth rate of the plant-pollinator patches is:

$\frac{d p_{2 L}}{d t} \frac{1}{p_{2 L}}=c_{2 L} p_{1 L}\left(1+I \frac{p_{2 M}}{p_{2 L}}\right)-e_{2 L}$

eqn 4

When $I=0$, per capita growth rate is independent of $p_{2 L}$, the fraction of plant-pollinator patches in the focal

(C) 2004 British

Ecological Society, Journal of Animal Ecology, 73,

128-142 locality. When $I>0$, per capita growth rate is a monotonic decreasing function of $p_{2 L}$ (Fig. 3). Because pollinator dispersal rate is independent of pollinator abundance in the focal locality, the per capita growth rate is high when local pollinator abundance is low and vice versa. Negative density-dependence induced by pollinator dispersal counteracts the positive density-dependence at low plant and pollinator abundances. This eliminates the Allee effect and allows species to increase when rare.

The idea of a mainland source that is unaffected by its interaction with a locality, while plausible, is perhaps not the most representative spatial structure. A metacommunity of several localities linked by dispersal represents a more realistic situation where the dynamics of each locality, source or sink, are affected by its interaction with other localities. I next investigate this situation.

\section{Several localities interconnected by dispersal}

Consider a collection of localities linked by dispersal of the mobile mutualist. There are two basic modes of dispersal. The first involves 'surplus' individuals from 
132

P. Amarasekare

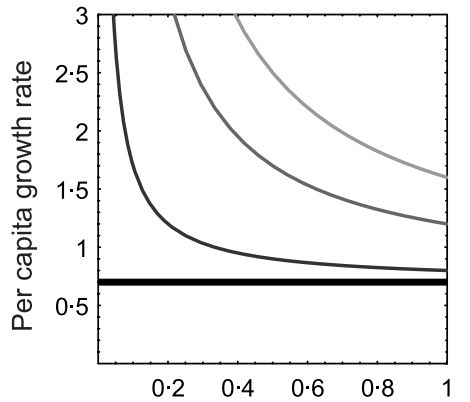

Abundance of plant-pollinator patches in locality $j\left(p_{2 j}\right)$

Fig. 4. Per capita growth rate of the plant-pollinator patches as a function of their abundance for the two modes of pollinator dispersal. The left panel represents dispersal of surplus individuals between localities, and the right panel shows dispersal of reproductive individuals between localities. In each panel, the horizontal line is the per capita growth rate in an isolated locality. The curves depict the per capita growth rate when the focal locality is linked to a source, with decreasing concavity corresponding to increasing abundance of plant-pollinator patches in the source locality $\left(p_{2 k}=0 \cdot 1,0 \cdot 5,0 \cdot 9\right)$. When dispersal involves surplus individuals, per capita growth rate is always augmented above that in isolation. When dispersal involves reproductive individuals, per capita growth rate can be increased or decreased relative to that in isolation, depending on the relative abundance of plantpollinator patches in source and sink localities. Parameter values are: $e_{2 j}=0 \cdot 3, h_{j}=0.5$ with $c_{2 j}=2$ for dispersal of surplus individuals and $c_{2 j}=3$ for dispersal of reproductive individuals.

one locality that colonize plant patches in other localities. These are individuals that moved too far out of their 'natal' locality, and would have died had they not found another locality. They could also be individuals that are forced out due to strong competition for colonizable patches within the natal locality. The point to note is that the loss of these surplus individuals does not affect local dynamics of the source locality.

The second dispersal mode involves emigration of 'reproductive' individuals. A fraction of propagules leave rather than attempt to colonize plants within their natal locality. Because emigrants are individuals that would otherwise have reproduced in situ, this mode of dispersal has a direct negative effect on local dynamics of the source locality. Holt $(1985,1993)$ and Pulliam (1988) have previously investigated the consequences of surplus vs. reproductive dispersal on single species populations. Here I consider the impact of these two modes of dispersal on mutualistic dynamics.

The distinction between the two modes of dispersal is easiest to illustrate with the appropriate equations. In the interests of analytical tractability, only two localities are considered.

The dynamics when pollinator dispersal involves surplus individuals are given by:

$\frac{d p_{1 j}}{d t}=c_{1 j} p_{2 j}\left(h_{j}-p_{1 j}-p_{2 j}\right)-c_{2 j} p_{1 j}\left(p_{2 j}+\frac{c_{2 k}}{c_{2 j}} p_{2 k}\right)-e_{1 j} p_{1 j}$

$\frac{d p_{2 j}}{d t}=c_{2 j} p_{1 j}\left(p_{2 j}+\frac{c_{2 k}}{c_{2 j}} p_{2 k}\right)-e_{2 j} p_{2 j}$

eqn 5

C) 2004 British Ecological Society, Journal of Animal Ecology, 73, 128-142 where $j$ and $k$ denote the two localities $(j, k=1,2, j \neq k)$, and other parameters are as defined above. The total amount of suitable habitat within the region (i.e. the spatial extent of the landscape that contains all localities) is assumed to be constant, and hence $h_{j}+h_{k}=1$. Note that the model is structurally similar to the mainland island case discussed above (equation 3 ) with $I=\frac{c_{2 k}}{c_{2 j}}$, the difference being that the abundance of plantpollinator patches in the source locality $\left(p_{2 k}\right)$ is now variable rather than constant.

The dynamics when pollinator dispersal involves reproductive individuals are given by:

$$
\begin{aligned}
\frac{d p_{1 j}}{d t}= & c_{1 j} p_{2 j}\left(h_{j}-p_{1 j}-p_{2 j}\right) \\
& -c_{2 j} p_{1 j}\left((1-d) p_{2 j}+d p_{2 k}\right)-e_{1 j} p_{1 j} \\
\frac{d p_{2 j}}{d t}= & c_{2 j} p_{1 j}\left((1-d) p_{2 j}+d p_{2 k}\right)-e_{2 j} p_{2 j}
\end{aligned}
$$

where $d$ is the fraction of pollinator individuals that emigrate from their natal locality.

The two modes of pollinator dispersal have very different effects on within-locality dynamics. When between-locality dispersal involves surplus individuals, the per capita growth rate of plant-pollinator patches is always augmented above that in isolation. For instance, the per capita growth rate when locality $j$ is isolated is $c_{2 j} p_{1 j}-e_{2 j}$; when connected by dispersal it is $c_{2 j} p_{1 j}\left(1+\frac{c_{2 k}}{c_{2 j}} \frac{p_{2 k}}{p_{2 j}}\right)-e_{2 j}$ (Fig. 4). Hence, betweenlocality dispersal of surplus individuals always has a positive effect on within-locality dynamics.

When between-locality dispersal involves reproductive individuals, the per capita growth rate of plant-pollinator patches may increase or decrease depending on their relative abundance in the two localities. For instance, if losses due to emigration from locality $j$ outweigh gains due to immigration from locality $k$, growth rate within locality $j$ can fall below that which it experiences in isolation 
133

Spatial dynamics of mutualisms $\left(c_{2 j} p_{1 j}\left((1-d) p_{2 j}+d \frac{p_{2 k}}{p_{2 j}}\right)-e_{2 j}\right.$ vs. $c_{2 j} p_{1 j}-e_{2 j}$; Fig. 4$)$.

Hence, dispersal of reproductive individuals can have a positive or negative effect on within-locality dynamics depending on whether the locality is a source or a sink.

As I show below, this difference between the two modes of dispersal is crucial in mitigating the Allee effect and allowing regional persistence of the mutualistic interaction.

\section{Dispersal of surplus individuals between localities}

Dynamics within each isolated locality follow equation 1, with two stable equilibria (extinction and coexistence) separated by the Allee threshold. Dispersal of surplus individuals does not change the basic dynamical structure of the model in that the threshold effect still persists (Appendix I). This is because, unlike in the mainland-island case (equation 3), the abundance of plant-pollinator patches in the source locality $\left(p_{2 k}\right)$ is not constant. Rather, it is a dynamic variable which itself is affected by both within-locality dynamics and between-locality dispersal.

While between-locality dispersal of surplus individuals does not eliminate the Allee effect within localities, it can mitigate it. As long as the abundance of plant and plant-pollinator patches are above the Allee threshold in one locality (source community, with positive per capita growth rate), pollinators can invade and establish in another locality in which both plant and plant-pollinator patches are below the Allee threshold (sink community, with negative per capita growth rate). Negative density-dependence due to the input of colonists from the source locality overwhelms the positive density-dependence within the sink locality (Fig. 5), allowing pollinators to invade when rare. Because between-locality dispersal involves surplus

\section{Dispersal of surplus individuals}
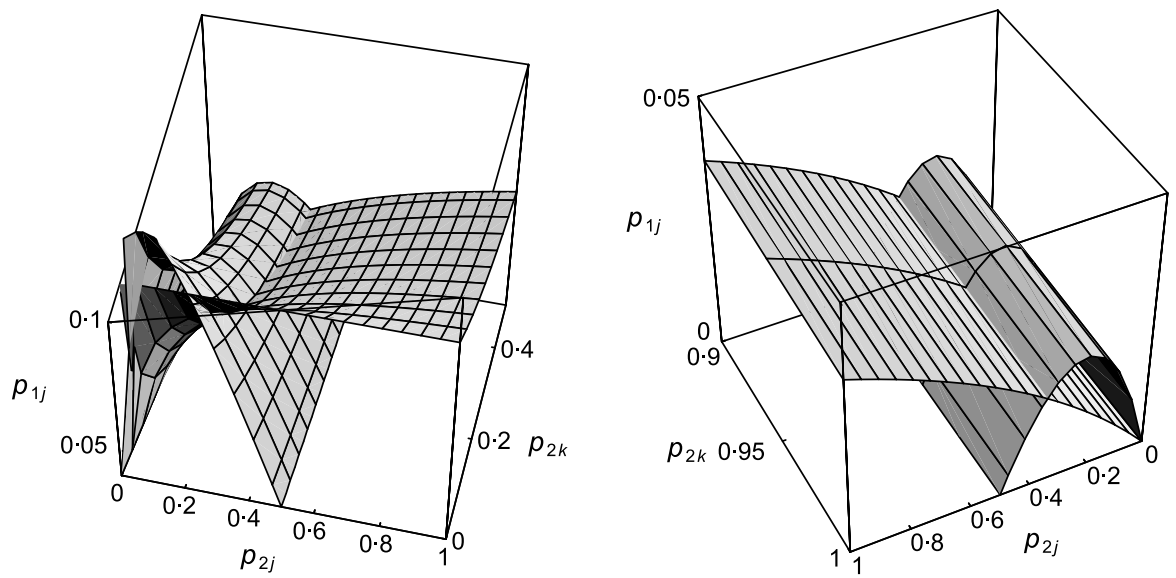

Dispersal of reproductive individuals
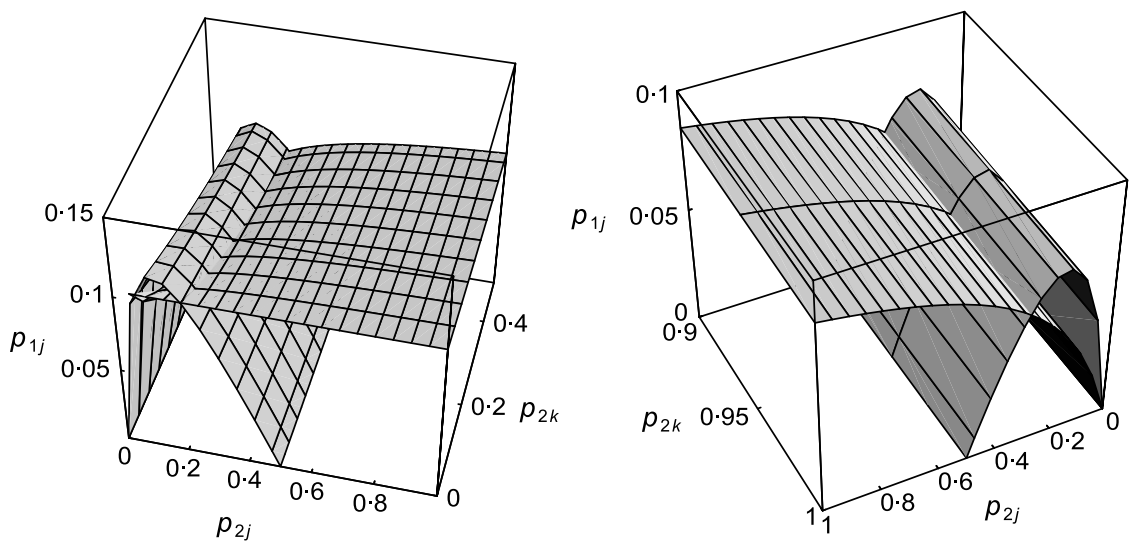

(C) 2004 British

Ecological Society, Journal of Animal Ecology, 73,

$128-142$
Fig. 5. Phase plots for two localities interconnected by dispersal. The $3 \mathrm{D}$ hyperbola is the zero isocline for plant patches, and the $3 \mathrm{D}$ surface, the zero isocline for plant-pollinator patches. When the abundance of plant-pollinator patches in the source locality is low ( $p_{2 k}=(0,0 \cdot 5)$; top left and bottom left panels), the Allee effect persists for both modes of dispersal, i.e. the isocline for plantpollinator patches crosses that of the plant patches twice, leading to multiple attractors (extinction and coexistence) separated by a saddle (compare with Fig. 1). When the abundance of plant-pollinator patches in the source locality is high $\left(p_{2 k}=(0 \cdot 9,1 \cdot 0)\right.$; top right and bottom right panels), negative density-dependence in dispersal overwhelms the Allee effect for both modes of dispersal, leading to stable coexistence of plant and pollinator in the sink locality. Parameter values are: $c_{1 j}=4, c_{1 k}=3, c_{2 j}=6, c_{2 k}=8, e_{i j}=0 \cdot 5, h_{j}=0 \cdot 5, d=0 \cdot 2$. 
individuals that do not affect local dynamics within the source, the per capita growth rate of the sink locality is augmented without an undue effect on that of the source locality.

The model of dispersal of surplus individuals (equation 5) assumes that a given pollinator individual has the same probability of colonizing plant patches in a new locality as it does within its natal locality. In nature, however, long-distance dispersers may suffer a mortality cost. Hence, not all surplus individuals that leave one locality may reach another one. If one assumes such mortality to be density-independent, then the per capita growth rate of plant-pollinator patches that receives colonists is:

$\frac{d p_{2 j}}{d t} \frac{1}{p_{2 j}}=c_{2 j} p_{1 j}\left(1+s \frac{c_{2 k}}{c_{2 j}} \frac{p_{2 k}}{p_{2 j}}\right)-e_{2 j}$ eqn 7

where $s$ is the fraction of long-distance dispersers from locality $k$ that survive to reach locality $j$.

It can be shown (Appendix I) that pollinator invasion when rare requires

$$
\begin{array}{r}
s>s_{\text {min }}=\left[e _ { 1 k } e _ { 2 k } \left(c_{1 j}^{2}\left(e_{2 j}-c_{2 j} h_{j}\right)^{2}+c_{2 j} e_{2 j}\left(c_{2 j} e_{2 j}+A\right)+\right.\right. \\
\frac{\left.\left.c_{1 j}\left(-2 c_{2 j}^{2} e_{2 j} h_{j}+e_{2 j} A-c_{2 j}\left(4 e_{1 j} e_{2 j}-2 e_{2 j}^{2}+h_{j} A\right)\right)\right)\right]}{2 c_{2 j} e_{1 j} e_{2 j}\left(e_{2 k}-c_{1 k}\left(1-h_{j}\right) A\right)}
\end{array}
$$

eqn 8

where $A=\sqrt{\left(c_{1 j}\left(e_{2 j}-c_{2 j} h_{j}\right)+c_{2 j} e_{2 j}\right)^{2}-4 c_{1 j} c_{2 j} e_{1 j} e_{2 j}}$, and $j, k=1,2, j \neq k$.

In biological terms this means the fraction of surplus individuals that survive long-distance dispersal has to be above a certain minimum in order for a sink community to be maintained via input from a source locality. This threshold is sensitive to both plant and pollinator colonization rate $\left(c_{1 j}\right.$ and $\left.c_{2 j}\right)$, and the amount of suitable habitat in the source locality $\left(h_{k}\right)$ (Fig. 6).

The key results are as follows: dispersal of surplus individuals between localities can mitigate the Allee effect and allow pollinators to invade and establish in localities that are empty or have a negative per capita growth rate. Thus, source-sink dynamics can allow both local and regional persistence of the mutualistic interaction. Source-sink dynamics have two important requirements. First, there has to be spatial heterogeneity in the environment such that at least one locality has plant and pollinator abundances above the Allee threshold. Secondly, the fraction of long-distance dispersers that survive to reach another locality has to exceed a critical threshold.

\section{Dispersal of reproductive individuals between localities}

(C) 2004 British Ecological Society, Journal of Animal Ecology, 73, $128-142$
Between-locality dispersal of reproductive individuals does not alter the basic dynamical structure of the model in that the within-locality Allee effect still persists (Appendix II). However, the Allee effect can be mitigated locally (Fig. 5).
In Appendix II I show that the pollinator can invade when rare if:

$$
\begin{array}{r}
d>d_{\text {min }}=\left[e _ { 1 k } e _ { 2 k } \left(c_{1 j}^{2}\left(e_{2 j}-c_{2 j} h_{j}\right)^{2}+c_{2 j} e_{2 j}\left(c_{2 j} e_{2 j}+A\right)+\right.\right. \\
\frac{\left.\left.c_{1 j}\left(-2 c_{2 j}^{2} e_{2 j} h_{j}+e_{2 j} A-c_{2 j}\left(4 e_{1 j} e_{2 j}-2 e_{2 j}^{2}+h_{j} A\right)\right)\right)\right]}{2 c_{2 k} e_{1 j} e_{2 j}\left(e_{2 k}-c_{1 k}\left(1-h_{j}\right) A\right)}
\end{array}
$$

where $A=\sqrt{\left(c_{1 j}\left(e_{2 j}-c_{2 j} h_{j}\right)+c_{2 j} e_{2 j}\right)^{2}-4 c_{1 j} c_{2 j} e_{1 j} e_{2 j}}$.

In biological terms this means the fraction of dispersers has to exceed a certain minimum in order for a sink community to be maintained via pollinator dispersal. Note that when plant and pollinator colonization rates are the same across localities (i.e. $c_{1 j}=c_{1 k}=c_{1}$ and $\left.c_{2 j}=c_{2 k}=c_{2}\right)$, the lower dispersal threshold $\left(d_{\text {min }}\right)$ is the same as that for pollinator colonization between localities $\left(s_{\min }\right)$. The magnitude of the threshold depends on both plant and pollinator colonization rates, and the amount of suitable habitat in the source locality (Fig. 6). Once $d$ exceeds this threshold, negative density-dependence due to dispersal counteracts the Allee effect and allows pollinators to increase when rare (Fig. 5).

These results parallel those obtained above when pollinator dispersal involves surplus individuals. There is, however, one critical difference between the two modes of dispersal.

When between-locality dispersal involves reproductive individuals, there is an upper dispersal threshold $\left(d_{\max }\right)$. The upper threshold arises because unlike with surplus individuals, dispersal of reproductive individuals can depress the per capita growth rate of the source locality. A fraction of pollinator individuals that would otherwise have reproduced within their natal locality leaves for another locality. If emigration is so high that the rate of production of plant-pollinator patches falls below the local extinction rate, the source community itself will have a negative per capita growth rate. This will cause region-wide extinction of both plant and pollinator.

The upper dispersal threshold cannot be calculated analytically, but numerical analyses show it to be sensitive to plant and pollinator colonization rates, and the amount of suitable habitat in the source locality (Fig. 6).

Incorporation of dispersal mortality does not alter the qualitative nature of source-sink dynamics when dispersal involves reproductive individuals. It does have the quantitative effect of increasing the lower dispersal threshold and lowering the upper dispersal threshold, thus making conditions for coexistence more restrictive.

The key results are as follows: dispersal of reproductive individuals can mitigate the Allee effect and allow region-wide persistence of the plant-pollinator interaction, provided the fraction emigrating is between a critical minimum and maximum. Unlike with dispersal of surplus individuals, too much emigration can cause 
Spatial dynamics of mutualisms
(C) 2004 British Ecological Society, Journal of Animal Ecology, 73 $128-142$
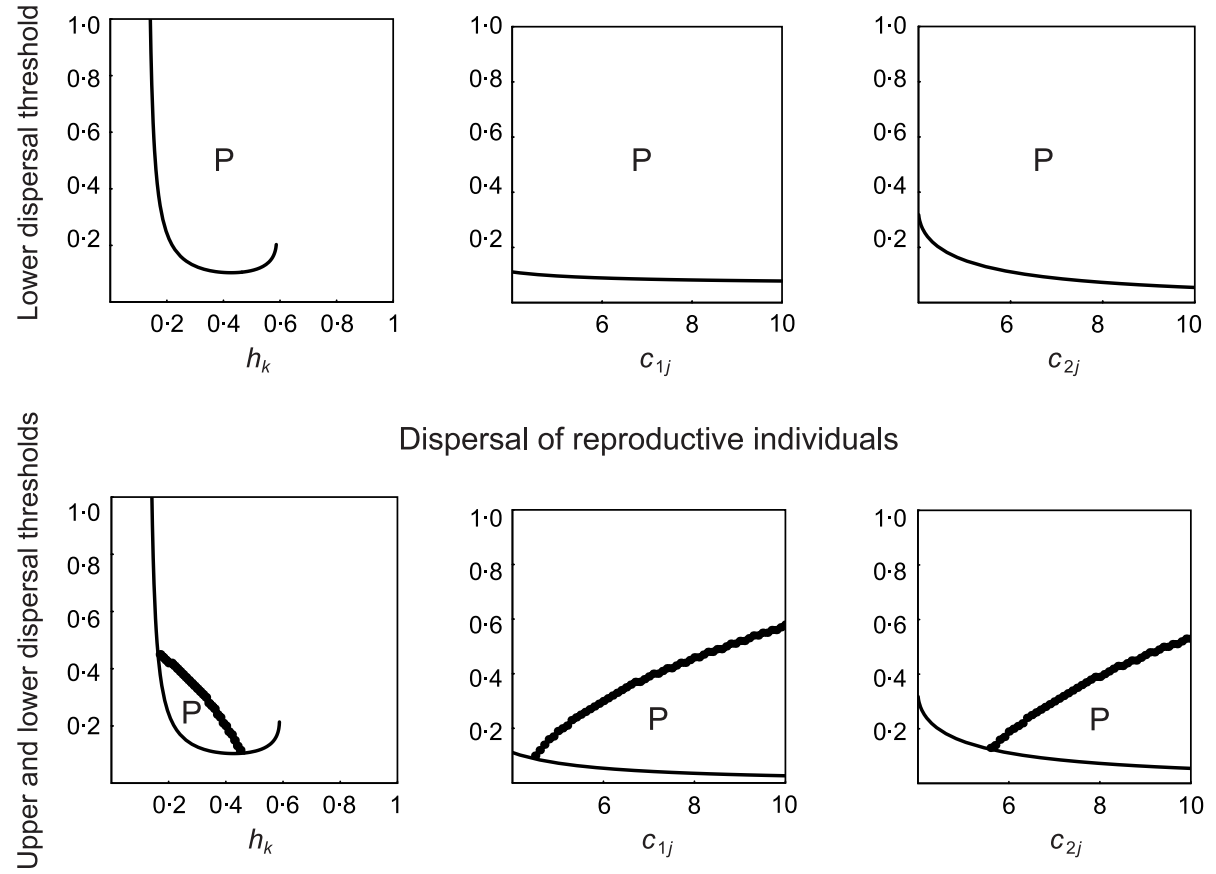

Dispersal of reproductive individuals
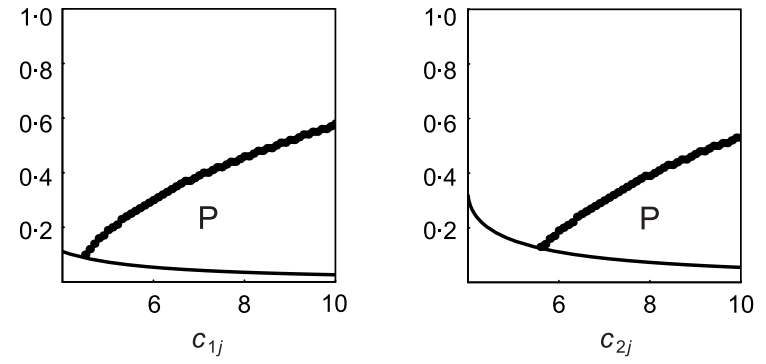

Fig. 6. Sensitivity of the lower and upper dispersal thresholds to the amount of suitable habitat in the source locality $\left(h_{k}\right)$ and colonization rates of plant and pollinator $\left(c_{1 j}\right.$ and $c_{2 j}$, respectively). The top three panels represent dispersal of surplus individuals, and the bottom three represent dispersal of reproductive individuals. The thin solid line represents the lower dispersal threshold, while the thick dotted line represents the upper threshold. The letter $P$ represents the region of the parameter space within which the mutualistic interaction can persist both locally and regionally. For both modes of dispersal, the lower dispersal threshold decreases (i.e. invasion by the mobile mutualist becomes easier) with increases in source habitat size and colonization rates of plant and pollinator. The lower threshold is more sensitive to the size of the source habitat than it is to the colonization rates. In the case of dispersal of reproductive individuals, the upper dispersal threshold increases with increasing colonization rates, thus increasing the region of the parameter space within which the interaction can persist. The upper threshold declines with increasing source habitat size, because the larger the source the greater the number of reproductive individuals emigrating and hence greater the likelihood that the per capita growth rate of the source becomes negative. Parameter values are: $c_{1 j}=c_{1 k}=4, c_{2 j}=c_{2 k}=6, e_{i j}=0 \cdot 5$, with $c_{1 k}=4, h_{j}=0.5$ for the middle panels, and $c_{2 k}=6, h_{j}=0.5$ for the right panels.

the per capita growth rate of the source locality to become negative, resulting in region-wide extinction of the mutualistic interaction.

\section{The role of habitat destruction}

Habitat destruction can be incorporated to the model by considering the total amount of available habitat within the region to be variable such that $h_{t}=h_{j}+h_{k}$ $\left(0<h_{t} \leq 1\right)$ where $h_{j}$ and $h_{k}$ are, respectively, the amount of habitat available in localities $j$ and $k$.

The role of habitat destruction within an isolated locality was analysed previously by Nee et al. (1997). Habitat destruction can have a large qualitative effect in that when $h_{j}$ falls below a critical threshold the two coexistence equilibria disappear, making extinction the only stable outcome.

Habitat destruction has no qualitative effect on metacommunity dynamics. For instance, as long as plant and pollinator abundance and suitable habitat area in one locality are above the extinction threshold, reducing the total habitat area $h_{t}$ does not cause global extinction of the mutualistic interaction. It does, however, make invasibility more difficult by increasing the magnitude of the lower dispersal threshold. This effect is the same for both modes of dispersal (Fig. 7). When dispersal involves reproductive individuals, habitat destruction has the additional effect of lowering the upper dispersal threshold and making conditions for persistence more restrictive.

\section{Distinguishing between the two modes of pollinator dispersal}

As the above results show, the two modes of pollinator dispersal can have quite different effects on the regional persistence of mutualistic interactions. An important issue concerns the biological significance of the two dispersal modes, particularly whether it is possible to distinguish between them in practice.

One way to distinguish between the two modes of dispersal is based on life history characteristics. Between-locality dispersal of surplus individuals may be more likely in species that engage in territoriality or other forms of interference competition (Pulliam 1988; Holt 1993) and those in which dispersal occurs in the adult stage and usually prior to reproduction (e.g. adult females of insect pollinators). Between-locality dispersal 
136

P. Amarasekare
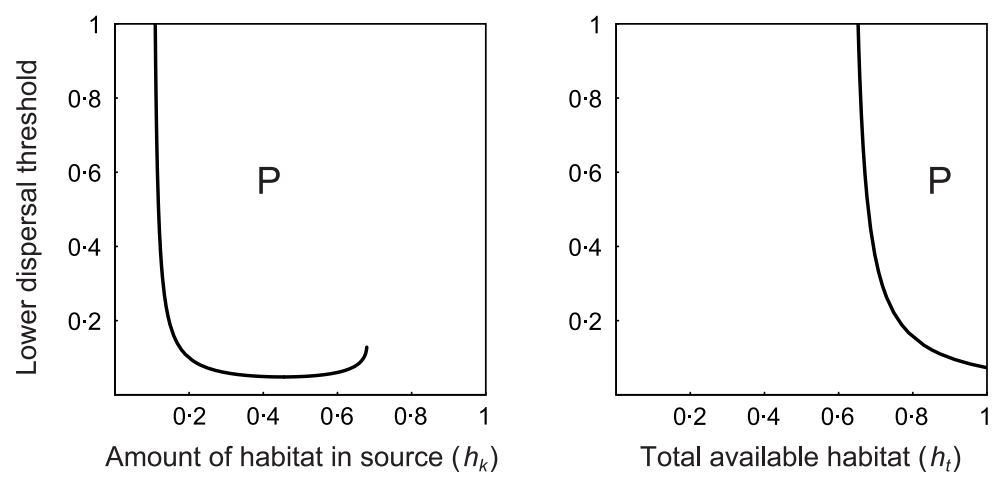

Fig. 7. The effect of habitat destruction on the lower dispersal threshold. In the absence of destruction (left panel) the threshold declines sharply as the amount of colonizable habitat in the source locality increases. Under habitat destruction, the threshold is shifted to the right, making invasion difficult even when survivorship of long-distance dispersers approaches $100 \%$ unless the total habitat available is quite large. Parameter values: $c_{1 j}=c_{1 k}=5, c_{2 j}=c_{2 k}=8, e_{i j}=0 \cdot 5, h_{j}=0 \cdot 5$.

Table 1. Comparative predictions for the persistence of mutualistic interactions under different types of population structure and levels of dispersal

\begin{tabular}{|c|c|c|c|}
\hline & No dispersal & Low dispersal & High dispersal \\
\hline \multirow[t]{2}{*}{$\begin{array}{l}\text { Dispersal from } \\
\text { mainland source }\end{array}$} & Allee effect & $\begin{array}{l}\text { No Allee effect } \\
\text { if } I>0\end{array}$ & $\begin{array}{l}\text { No Allee effect } \\
\text { if } I>0\end{array}$ \\
\hline & Local extinction & Regional persistence & Regional persistence \\
\hline $\begin{array}{l}\text { Dispersal of } \\
\text { surplus individuals }\end{array}$ & Allee effect & $\begin{array}{l}\text { Allee effect mitigated } \\
\text { if } s_{\min }<s\end{array}$ & $\begin{array}{l}\text { Allee effect mitigated } \\
\text { if } s_{\min }<s\end{array}$ \\
\hline between localities & Local extinction & Regional persistence & Regional persistence \\
\hline $\begin{array}{l}\text { Dispersal of } \\
\text { reproductive individuals }\end{array}$ & Allee effect & $\begin{array}{l}\text { Allee effect mitigated } \\
\text { if } d_{\min }<d<d_{\max }\end{array}$ & $\begin{array}{l}\text { Allee effect } \\
\text { if } d>d_{\max }\end{array}$ \\
\hline between localities & Local extinction & Regional persistence & Regional extinction \\
\hline
\end{tabular}

of reproductive individuals may be more prevalent in species in which the juvenile stages tend to disperse. Examples include rodents, many of whom serve as seed dispersers, and birds.

Regardless of the life history strategy, the crucial distinguishing factor is the difference in the per capita growth rates of plant-pollinator patches. This difference can be quantifed by comparing growth rates of isolated localities with those linked by dispersal (interlocality distance could in many cases serve as a surrogate for the degree of connectivity between localities).

If dispersal involves surplus individuals that cannot reproduce within their natal locality, per capita growth rates of connected localities should always exceed those of isolated localities. In contrast, if dispersal involves exchanging a fraction of the local reproductive output from each locality, per capita growth rates of connected localities may be higher or lower than those of isolated localities, depending on the relative abundance of plant-pollinator patches in the localities being compared. If data are available for more than one time period, repeating the analysis over time can determine whether the growth rate changes sign or remains positive.

Table 1 lists a set of comparative predictions about how population structure and modes of dispersal influence the persistence of the mutualistic interaction.

\section{MUTUALISTIC DYNAMICS WHEN ONE SPECIES IS FACULTATIVE}

The above results were obtained when both interacting species were obligate mutualists. An interesting question is how these results may be altered if one species is a facultative mutualist.

\section{Mobile species is a generalist}

Plant species dependent on generalist pollinators or seed dispersers are common in nature. Examples include plants pollinated by honeybees or bumble bees, and whose seeds are dispersed by rodents (Howe \& Smallwood 1982; Feinsinger 1983; Herrera 1984, 1988; Bond 1994).

The above model (equation 2) can be interpreted as that of a generalist pollinator or seed disperser that services two plant species. Each locality then represents a distinct plant species that is patchily distributed. As long as the pollinator or seed disperser can maintain a positive per capita growth rate on one species of plant, it can rescue from extinction another plant species that experiences an Allee effect. If the plant species are congeners or otherwise closely related, pollinator movement between localities may have the adverse effect of cross-pollination and hybridization (see Discussion). 
137

Spatial dynamics of mutualisms
(C) 2004 British Ecological Society, Journal of Animal Ecology, 73, $128-142$

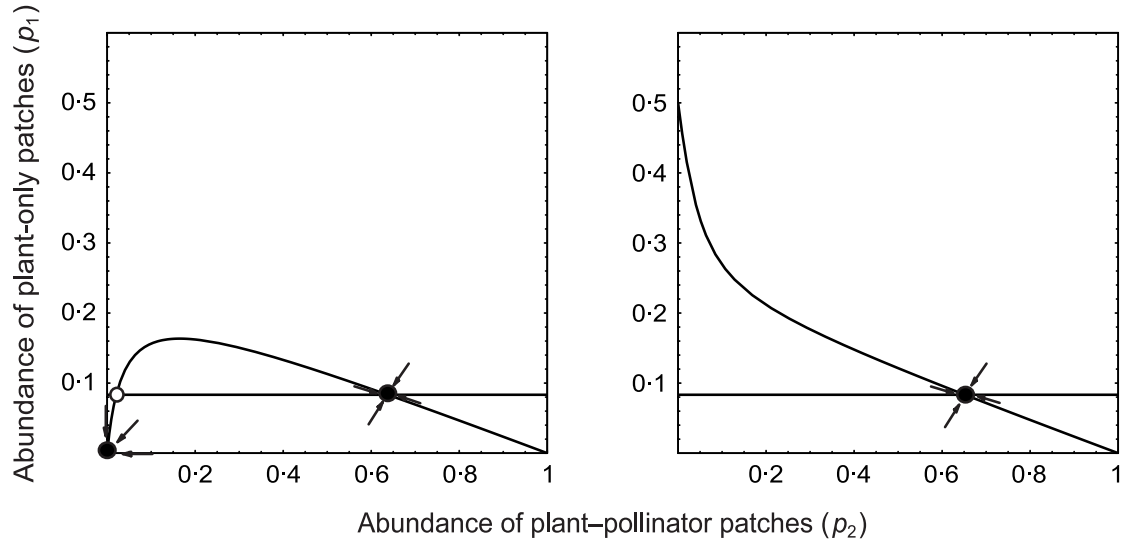

Fig. 8. Phase plots when the plant is a facultative mutualist and the pollinator is an obligate mutualist. If plant reproduction is depressed sufficiently in the absence of the specialist pollinator, the Allee effect persists $(m=0 \cdot 1$; left panel). If the plant can reproduce sufficiently well in the absence of the specialist pollinator $(m=0 \cdot 5$; right panel), stable coexistence is possible even within an isolated locality. Parameter values: $c_{1}=2, c_{2}=6, e_{1}=e_{2}=0 \cdot 5, h=1$.

\section{Non-mobile species is a generalist}

Consider the case when the plant species is a generalist and the pollinator is an obligate mutualist. Now plants can reproduce even in the absence of the specialist pollinator, although seed set may be depressed in such patches. This scenario leads to the following model for an isolated locality:

$\frac{d p_{1}}{d t}=\left(c_{1} p_{2}+m c_{1} p_{1}\right)\left(h-p_{1}-p_{2}\right)-c_{2} p_{1} p_{2}-e_{1} p_{1}$

$\frac{d p_{2}}{d t}=c_{2} p_{1} p_{2}-e_{2} p_{2}$

eqn 10

with $m$ representing the reduction in seed set and propagule establishment in the absence of the specialist pollinator $(0<m \leq 1)$.

Equation 10 admits four equilibria: the trivial equilibrium with both species extinct $\left(p_{1}^{*}, p_{2}^{*}\right)=(0,0)$, a boundary equilibrium with the pollinator extinct $\left(p_{1}^{*}, p_{2}^{*}\right)$ $=\left(h-\frac{e_{1}}{c_{1} m}, 0\right)$ and two interior equilibria $\left(p_{1}^{*}, p_{2}^{*}\right)=$ $\left(\frac{e_{2}}{c_{2}}, \frac{c_{2}^{2}\left(c_{1} h-e_{2}\right)-c_{1} c_{2} e_{2}(1+m) \pm \sqrt{c_{2}^{2}\left(c_{1} h-e_{2}\right)-c_{1} c_{2} e_{2}(1+m)^{2}-4 c_{1} c_{2}^{2} e_{2}\left(c_{1} m\left(e_{2}-c_{2} h\right)\right)}}{2 c_{1} c_{2}^{2}}\right)$. When $m<\frac{e_{1} c_{2}}{c_{1}\left(c_{2} h-e_{2}\right)}$, the Allee effect persists and the trivial equilibrium and the larger of the interior equilibria are simultaneously stable. (The boundary equilibrium with pollinator extinct is not feasible in the presence of an Allee effect.) When plant and pollinator abundances fall below the threshold defined by the smaller of the coexistence equilibria, both species go extinct. However, if $m>\frac{e_{1} c_{2}}{c_{1}\left(c_{2} h-e_{2}\right)}$, the Allee effect disappears and the only feasible equilibria are the boundary equilibrium and the larger of the interior equilibria. The former is unstable and the latter stable, thus allowing the two species to coexist (Fig. 8).
In biological terms, if the plant is able to reproduce sufficiently well in the absence of the specialist pollinator, then the positive density-dependence in its per capita growth rate disappears, and both plant and pollinator can coexist stably within an isolated locality. Pollinator dispersal between localities is not necessary for persistence of the mutualistic interaction. However, if plant reproduction in the absence of the specialist pollinator is depressed below the threshold defined above, then the Allee effect persists and extinction of both plant and pollinator can occur within an isolated locality. In this case, pollinator dispersal between localities becomes important. Dispersal of pollinators from source localities can rescue such sink communities from extinction. As before, the rescue effect depends on the mode of dispersal, with high dispersal of reproductive individuals being detrimental to persistence.

\section{Discussion}

Empirical studies suggest that mutualistic interactions in fragmented landscapes are likely to suffer from Allee effects (e.g. Jennersten 1988; Lamont et al. 1993; Aizen \& Feinsinger 1994; Agren 1996; Kunin \& Iwasa 1996; Groom 1998). Despite the obvious importance of how mutualisms may persist in fragmented landscapes, little or no theory exists on their spatial dynamics. Here I have presented a metacommunity model of a mutualistic interaction between two species that differ in their mobility. I show that spatial structure and dispersal can have important, and sometimes counterintuitive, consequences on the persistence of mutualistic interactions. Although I have described the model in terms of plant-pollinator interactions, these results apply to any mutualistic interaction in which the reproduction and establishment of a species that is relatively immobile depends on a species that is relatively mobile.

The key results of this study involve the roles of dispersal mode and dispersal-induced density-dependence on 
the regional persistence of mutualistic interactions. Although previous work has demonstrated the importance of these factors in single species populations (Holt 1985, 1993; Pulliam 1988), their impact on species interactions has not been explored. Here I show that in mutualistic interactions subject to Allee effects, dispersal induces negative density dependence in the per capita growth rate of the community that receives dispersers. This negative density-dependence overwhelms the positive density-dependence at low abundances.

Dispersal itself is density-independent, but it creates a negative feedback effect that increases the strength of intraspecific interactions (e.g. self-limitation) relative to that of interspecific interactions (e.g. Allee effect induced by mutualistic partner). This negative feedback allows both mutualists to increase when rare, thus preventing extinction of the mutualistic interaction in sink habitats.

The second key result concerns the effect of dispersal mode on the regional persistence of the mutualistic interaction. When dispersal involves surplus individuals the loss of whom does not affect the reproductive output of the source community, local and regional persistence are guaranteed as long as the fraction of long distance dispersers that survive to reach sink communities exceeds a lower threshold. In contrast, when dispersal involves individuals that constitute a fraction of the source community's reproductive output, maintenance of sink communities requires the fraction emigrating to be above a lower threshold, but not exceed an upper threshold. Too much dispersal causes a net loss of the mobile mutualist from source communities, leading to regional extinction of the entire mutualistic interaction.

While these results on the effect of dispersal mode parallel those obtained for single species source-sink dynamics (Holt 1985, 1993; Pulliam 1988), there is an important difference. Because mutualisms can generate Allee effects, dispersal losses are more likely to increase the extinction risk of source communities, and hence the risk of region-wide extinction, compared to single species or other species interactions such as competition or predation. The dispersal-induced extinction risk to source communities also distinguishes this study from previous work on Allee effects and dispersal in single species. (e.g. Lewis \& Kareiva 1993; Amarasekare 1998; Gyllenberg et al. 1999; Keitt et al. 2001). In these studies the Allee effect is considered only phenomenologically, i.e. the extinction threshold is a constant that is not affected by local population dynamics or dispersal. Hence, an increase in the dispersal rate does not affect the extinction probability of source populations. Rather, it allows both source and sink to persist at their respective carrying capacities (Amarasekare 1998; Gyllenberg et al. 1999). In the model presented here, the Allee extinction threshold arises as a direct consequence of the mutualistic interaction. It is therefore a dynamic threshold that depends both on local species interactions and dispersal. Hence, an increase in the dispersal rate can drive an otherwise viable source community to extinction by reducing its abundance below the Allee threshold.
The crucial implication of these results is that source-sink dynamics cannot guarantee the long-term persistence of mutualistic interactions. Persistence depends on the balance between the benefit of the rescue effect to sink communities, and the cost to source communities in terms of loss of reproductive output. The negative effect of dispersal on source communities should be an important consideration both in empirical studies of mutualistic interactions, and in developing management measures for preserving or restoring mutualisms in fragmented landscapes. In this respect, it is important to quantify not only the rate of dispersal but also the effect dispersal has on local population growth. This information can be obtained by measuring both the local reproductive rate and the per capita growth rate, as the latter encompasses local reproduction as well as losses due to dispersal. The magnitude of the local reproductive rate relative to the per capita growth rate can not only indicate the source-sink status of a given community, but also the effect dispersal has on local dynamics. For instance, a sink community that is being rescued is likely to have a low or negative local recruitment rate but a per capita growth rate that is positive, whereas a source community that suffers a cost due to dispersal is likely to have a positive local recruitment rate but a per capita growth rate that is low or negative. In cases where these vital rates cannot be measured, time-series of abundance data can be informative. A steady decline in the abundance of a putative source community could signify a negative per capita growth rate.

In terms of management measures, the cost of dispersal to source communities is the most crucial consideration. In species whose local reproduction is unaffected by dispersal, linking habitat fragments via dispersal corridors may be beneficial because sourcesink dynamics ensure both local and regional persistence. In species whose local reproduction is reduced by dispersal (e.g. birds and small mammals in which juvenile dispersal is the norm; Andreassen, Stenseth \& Ims 2002), dispersal rate should be sufficiently low that the growth rate of source communities are not depressed. Such species may also experience higher risk of largescale extinction because habitat destruction can induce mass emigration of individuals, leading to rapid extinction of source communities. In such cases preserving large habitat fragments, regardless of their connectivity, may be more conducive to preserving mutualisms than connecting fragments by dispersal.

The result that extinction risk due to Allee effects is greatest in obligate mutualisms raises the question of how Allee effects and habitat fragmentation impact facultative mutualisms. This is an important consideration given that a large number of mutualisms in nature tend to be facultative (Bond 1994; Hoeksema \& Bruna 2000). The model with dispersal between localities can in fact be interpreted as that of a generalist pollinator or seed disperser that services two plant species. In this case, provided the pollinator or seed disperser can 

of mutualisms maintain a positive growth rate on one plant species, it can prevent the other plant species from going extinct when rare. This type of rescue effect due to a generalist pollinator, however, can have adverse consequences for rare species that are surrounded by other flowering plants. Transfer of the wrong species' pollen can lead to reproductive failure of the rare species (Feinsinger, Tiebout \& Young 1991; Kunin 1993; Aizen \& Feinsinger 1994). Worse still, such transfers can lead to interspecific hybridization and the consequent genetic loss of rare or endangered species (Schemske et al. 1994; Levin, Francisco-Ortega \& Jansen 1996).

The extension of the model to a mutualism between an obligate mobile species and a facultative nonmobile species shows that Allee effects can be less extreme in such situations. Persistence via sourcesink dynamics is facilitated in this situation because localities in which the non-mobile species can reproduce sufficiently well in the absence of the mobile mutualist do not experience an Allee effect. Dispersal from such source communities can rescue those in which Allee effects prevail. Because there are communities that do not experience Allee effects at all, the cost of dispersal to sources is likely to be lower in this situation.

In conclusion, spatial dynamics can enhance or hinder the persistence of mutualistic interactions in fragmented landscapes, depending on the effect dispersal has on local reproduction of source communities. While low dispersal rates promote diversity by increasing the benefit of rescue effects to sinks relative to the reproductive cost to sources, high dispersal rates can constrain diversity by increasing costs relative to benefits.

\section{Acknowledgements}

This research was conducted as a part of the Metacommunity Working Group at the National Center for Ecological Analysis and Synthesis (NCEAS), a centre funded by the National Science Foundation (DEB9421535), University of California Santa Barbara, and the state of California. The author was supported by NSF grant DEB-0129270 and the Louise R. Block Fund from the University of Chicago. J. Bascompte, T. Knight and two anonymous referees provided many helpful comments on the manuscript.

\section{References}

Agren, J. (1996) Population size, pollinator limitation and seed set in the self-incompatible herb Lythrum salicaria. Ecology, 77, 1779-1790.

Aizen, M.A. \& Feinsinger, P. (1994) Forest fragmentation, pollination, and plant reproduction in a Chaco dry forest, Argentina. Ecology, 75, 330-351.

(C) 2004 British Ecological Society, Journal of Animal Ecology, 73, $128-142$
Andreassen, H.P., Stenseth, N.C. \& Ims, R.A. (2002) Dispersal behaviour and population dynamics of vertebrates. Dispersal Ecology, 1, 237-256.

Anstett, M., Hossaert-McKey, M. \& McKey, D. (1997). Modeling the persistence of small populations of strongly interdependent species: figs and fig wasps. Conservation Biology, 11, 204-213.

Armstrong, R.A. (1987) A patch model of mutualism. Journal of Theoretical Biology, 125, 243-246.

Bond, W.J. (1994) Do mutualisms matter? Assessing the impact of pollinator and disperser disruption on plant extinction. Philosophical Transactions of the Royal Society of London, 344, 83-90.

Cane, J.H. (2001) Habitat fragmentation and native bees: a premature verdict? Conservation Ecology, 5, 3. Available online at: URL: http://www.consecol.org/vol5/iss1/art3.

Dean, A.M. (1983) A simple model of mutualism. American Naturalist, 121, 409-417.

Dennis, B. (1989) Allee effects: population growth, critical density, and the chance of extinction. Natural Resource Modeling, 3, 481-538.

Feinsinger, P. (1983) Coevolution and pollination. Coevolution (eds D.J. Futuyma \& M. Latkin), pp. 282-310. Sinauer: Sunderland, MA.

Feinsinger, P., Tiebout, H.M. III \& Young, B.E. (1991) Do tropical bird-pollinated plants exhibit density-dependent interactions? Field experiments. Ecology, 72, 1953-1963.

Frankel-Conrat, H. \& Wagner, R.R. (1977) Plant Viruses. Comprehensive Virology Series. Plenum Press, New York.

Groom, M.J. (1998) Allee effects limit population viability of an annual plant. American Naturalist, 151, 487-496.

Gyllenberg, M., Hemminki, J. \& Tammaru, T. (1999) Allee effects can both conserve and create spatial heterogeneity in population densities. Theoretical Population Biology, 56, 231-242.

Herrera, C.M. (1984) A study of avian frugivores, bird-dispersed plants and their interaction in Mediterranean scrublands. Ecological Monographs, 54, 1-23.

Herrera, C.M. (1988) Variation in mutualisms: the spatiotemporal mosaic of a pollinator assemblage. Biology Journal of the Linnean Society, 35, 95-125.

Hoeksema, J.D. \& Bruna, E.M. (2000) Pursuing the big questions about interspecifc mutualism: a review of theoretical approaches. Oecologica, 125, 321-330.

Holt, R.D. (1985) Population dynamics in two-patch environments: some anomalous consequences of an optimal habitat distribution. Theoretical Population Biology, 28, 181208

Holt, R.D. (1993) Ecology at the mesoscale: the influence of regional processes on local communities. Species Diversity in Ecological Communities: Historical and Geographical Perspectives (eds R.E. Ricklefs \& D. Schluter), pp. 77-88. University of Chicago Press, Chicago.

Howe, H.F. \& Smallwood, J. (1982) Ecology of seed dispersal. Annual Review of Ecology and Systematics, 13, 201-228.

Hutson, V., Law, R. \& Lewis, D. (1985) Dynamics of ecologically obligate mutualisms - effects of spatial diffusion on resilience of the interacting species. American Naturalist, 126, 445-449.

Jennersten, O. (1988) Pollination in Dianthus deltoides (Caryophyllaceae): effects of habitat fragmentation on visitation and seed set. Conservation Biology, 2, 359-366.

Keitt, T.H., Lewis, M.A. \& Holt, R.D. (2001) Allee effects, invasion pinning, and species' borders. American Naturalist, 157, 203-216.

Kunin, W.E. (1993) Sex and the single mustard: population density and pollinator behavior effects on seed-set. Eco$\log y, \mathbf{7 4}, 2145-2160$. 
P. Amarasekare
Kunin, W.E. \& Iwasa, Y. (1996) Pollinator foraging strategies in mixed floral arrays: density effects and floral constancy. Theoretical Population Biology, 49, 232-263.

Lamont, B.B., Klinkhamer, P.G.L. \& Witkowski, E.T.F. (1993) Population fragmentation may reduce fertility to zero in Banksia goodii: a demonstration of the Allee effect. Oecologia, 94, 446-450.

Levin, D.A., Francisco-Ortega, J. \& Jansen, R.K. (1996) Hybridization and the extinction of rare plant species. Conservation Biology, 10, 10-16.

Lewis, M.A. \& Kareiva, P. (1993) Allee dynamics and the spread of invading organisms. Theoretical Population Biology, $\mathbf{4 3}$, 141-158.

May, R.M. (1978) Mathematical aspects of the dynamics of animal populations. Studies in Mathematical Biology (ed. S.A. Levin), pp. 317-366. Mathematical Association of America, Washington DC.

Nee, S., May, R.M. \& Hassell, M.P. (1997) Two species metapopulation models. Metapopulation Biology: Ecology, Genetics and Evolution (eds I. Hanski \& M. Gilpin), pp. 123 146. Academic Press, London.
Packer, L. \& Owen, R. (2001) Population genetic aspects of pollinator decline. Conservation Ecology, 5, 163-198.

Peden, K.W.C. \& Symons, R.H. (1976) Cucumber mosaic virus contains a functionally divided genome. Virology, 53, 487-492.

Pulliam, R. (1988) Sources, sinks and population regulation. American Naturalist, 132, 652-661.

Schemske, D.W., Husband, B.C., Ruckelshaus, M.H., Goodwillie, C., Parker, I.M. \& Bishop, J.G. (1994) Evaluating approaches to the conservation of rare and endangered plants. Ecology, 75, 584-606.

Vandermeer, J.H. \& Boucher, D.H. (1978) Varieties of mutualistic interaction in population models. Journal of Theoretical Biology, 74, 549-558.

Wells, H. (1983) Population equilbiria and stability in plantanimal pollination systems. Journal of Theoretical Biology, 100, 685-699.

Wolin, C.L. (1985) The population dynamics of mutualistic systems. The Biology of Mutualisms (ed. D.H. Boucher), pp. 248-269. Oxford University Press, New York.

Received 20 January 2003; accepted 22 July 2003
(C) 2004 British

Ecological Society, Journal of Animal Ecology, 73,

$128-142$ 
141

Spatial dynamics of mutualisms
(C) 2004 British

Ecological Society, Journal of Animal Ecology, 73,

$128-142$

\section{Appendix I}

EQUILIBRIA AND INVASION ANALYSIS FOR THE MODEL WITH DISPERSAL OF SURPLUS INDIVIDUALS BETWEEN LOCALITIES

In the absence of within-locality dispersal, the mutualistic interaction within each locality yields three equilibria:

1. $\left(p_{1 j}^{*}, p_{2 j}^{*}\right)_{I E}=(0,0)$

2. $\left(p_{1 j}^{*}, p_{2 j}^{*}\right)_{I T}=\left(\frac{e_{2}}{c_{2}}, \frac{c_{1}\left(c_{2} h-e_{2}\right)-c_{2} e_{2}-\sqrt{\left(c_{1}\left(c_{2} h-e_{2}\right)-c_{2} e_{2}\right)^{2}-4 c_{1} c_{2} e_{1} e_{2}}}{2 c_{1} c_{2}}\right)$

3. $\left(p_{1 j}^{*}, p_{2 j}^{*}\right)_{I C}=\left(\frac{e_{2}}{c_{2}}, \frac{c_{1}\left(c_{2} h-e_{2}\right)-c_{2} e_{2}+\sqrt{\left(c_{1}\left(c_{2} h-e_{2}\right)-c_{2} e_{2}\right)^{2}-4 c_{1} c_{2} e_{1} e_{2}}}{2 c_{1} c_{2}}\right)$

Only (1) and (3) are stable. Two isolated localities each admitting these three equilibria therefore have a total of nine possible equilibrium states (Table A1).

When the localities are linked by dispersal of surplus individuals, two of the nine equilibria disappear (Table A1). Higher colonization rates or lower dispersal mortality does not affect the remaining seven equilibria.

The Jacobain matrix of equation 5 is given by:

$$
\left[\begin{array}{cccc}
-\left(p_{21}^{*}\left(c_{11}+c_{21}\right)+P+e_{11}\right) & 0 & c_{11} Q-c_{21} p_{11}^{*} & -c_{22} p_{11}^{*} s \\
0 & -\left(p_{22}^{*}\left(c_{12}+c_{22}\right)+c_{21} p_{21}^{*} s+e_{12}\right) & -c_{21} p_{12}^{*} s & c_{12} R-S \\
c_{21} p_{21}^{*}+P & 0 & c_{21} p_{11}^{*}-e_{21} & c_{22} p_{11}^{*} s \\
0 & c_{22} p_{22}^{*}+c_{21} p_{21}^{*} s & c_{21} p_{12}^{*} s & S-e_{22}
\end{array}\right]
$$

where $P=c_{22} p_{22}^{*} s, Q=\left(h_{1}-p_{11}^{*}-2 p_{21}^{*}\right), R=\left(h_{2}-p_{12}^{*}-2 p_{22}^{*}\right)$ and $S=c_{22} p_{12}^{*}$.

The mobile mutualist can invade an initially empty locality if the dominant eigenvalue of the Jacobian is positive when evaluated at the equilibrium:

$\left(p_{1 j}^{*}=\frac{e_{2 j}}{c_{2 j}}, p_{2 j}^{*}=\frac{c_{1 j}\left(c_{2 j} h_{j}-e_{2 j}\right)-c_{2 j} e_{2 j}+\sqrt{\left(c_{1 j}\left(c_{2 j} h_{j}-e_{2 j}\right)-c_{2 j} e_{2 j}\right)^{2}-4 c_{1 j} c_{2 j} e_{1 j} e_{2 j}}}{2 c_{1 j} c_{2 j}}, p_{1 k}^{*}=0, p_{2 k}^{*}=0\right)$

Solving the dominant eigenvalue for $s$ leads to the invasion criterion (equation 8) in the main text.

Table A1. Equilibria when pollinator dispersal involves surplus individuals

\begin{tabular}{lll}
\hline Isolated & Low dispersal & High dispersal \\
\hline 1. $0,0,0,0$ & $0,0,0,0$ & $0,0,0,0$ \\
2. $0,0\left(p_{1 k}^{*}, p_{2 k}^{*}\right)_{I T}$ & $0,0\left(p_{1 k}^{*}, p_{2 k}^{*}\right)_{C T}$ & $0,0\left(p_{1 k}^{*}, p_{2 k}^{*}\right)_{C T}$ \\
3. $0,0\left(p_{1 k}^{*}, p_{2 k}^{*}\right)_{I C}$ & $0,0\left(p_{1 k}^{*}, p_{2 k}^{*}\right)_{C C}$ & $0,0\left(p_{1 k}^{*}, p_{2 k}^{*}\right)_{C C}$ \\
4. $\left(p_{1 j}^{*}, p_{2 j}^{*}\right)_{I T}, 0,0$ & $\left(p_{1 j}^{*}, p_{2 j}^{*}\right)_{C T}, 0,0$ & $\left(p_{1 j}^{*}, p_{2 j}^{*}\right)_{C T}, 0,0$ \\
5. $\left(p_{1 j}^{*}, p_{2 j}^{*}\right)_{I C}, 0,0$ & $\left(p_{1 j}^{*}, p_{2 j}^{*}\right)_{C C}, 0,0$ & $\left(p_{1 j}^{*}, p_{2 j}^{*}\right)_{C C}, 0,0$ \\
6. $\left(p_{1 j}^{*}, p_{2 j}^{*}\right)_{I T},\left(p_{1 j}^{*}, p_{2 j}^{*}\right)_{I C}$ & \\
7. $\left(p_{1 j}^{*}, p_{2 j}^{*}\right)_{I C},\left(p_{1 j}^{*}, p_{2 j}^{*}\right)_{I T}$ & & \\
8. $\left(p_{1 j}^{*}, p_{2 j}^{*}\right)_{I T},\left(p_{1 k}^{*}, p_{2 k}^{*}\right)_{I T}$ & & $\left(p_{1 j}^{*}, p_{2 j}^{*}\right)_{C T},\left(p_{1 k}^{*}, p_{2 k}^{*}\right)_{C T}$ \\
9. $\left(p_{1 j}^{*}, p_{2 j}^{*}\right)_{I C},\left(p_{1 k}^{*}, p_{2 k}^{*}\right)_{I C}$ & $\left(p_{1 j}^{*}, p_{2 j}^{*}\right)_{C T},\left(p_{1 k}^{*}, p_{2 k}^{*}\right)_{C T}$ & $\left(p_{1 j}^{*}, p_{2 j}^{*}\right)_{C C},\left(p_{1 k}^{*}, p_{2 k}^{*}\right)_{C C}$
\end{tabular}

IT $=$ isolated, threshold $; \mathrm{IC}=$ isolated coexistence $; \mathrm{CT}=$ connected, threshold $; \mathrm{CC}=$ connected, coexistence . 
142

P. Amarasekare

\section{Appendix II}

\section{INVASION ANALYSIS FOR THE MODEL WITH DISPERSAL OF REPRODUCTIVE INDIVIDUALS} BETWEEN LOCALITIES

Table (A2) characterizes the equilibria for isolated localities vs. those linked by dispersal. When localities are linked by dispersal of reproductive individuals, all nine equilibria persist. When emigration exceeds the upper threshold, six of the nine equilibria disappear. The remaining three equilibria correspond to extinction, Allee threshold and coexistence in both localities.

The Jacobain matrix of equation 6 is given by:

$$
\left[\begin{array}{cccc}
-\left(p_{21}^{*}\left(c_{11}+c_{21}\right)+c_{21} d P+e_{11}\right) & 0 & c_{11} Q-c_{21} p_{11}^{*}(1-d) & -c_{22} p_{11}^{*} d \\
0 & -\left(p_{22}^{*}\left(c_{12}+c_{22}\right)+c_{22} d P+e_{12}\right) & -c_{22} p_{12}^{*} d & c_{12} R-S \\
c_{21}\left(p_{21}^{*}(1-d)+d p_{22}^{*}\right) & 0 & c_{22} p_{11}(1-d)-e_{21} & c_{22} p_{11} d \\
0 & c_{22}\left(p_{22}^{*}(1-d)+d p_{21}^{*}\right) & c_{22} p_{12}^{*} d & S-e_{22}
\end{array}\right]
$$

where $P=\left(p_{21}^{*}+p_{22}^{*}\right), Q=\left(h_{1}-p_{11}^{*}-2 p_{21}^{*}\right), R=\left(h_{2}-p_{12}^{*}-2 p_{22}^{*}\right)$ and $S=c_{22} p_{12}^{*}(1-d)$.

The mobile mutualist can invade an initially empty locality if the dominant eigenvalue of the Jacobian is positive when evaluated at the equilibrium

$$
\left(p_{1 j}^{*}=\frac{e_{2 j}}{c_{2 j}}, p_{2 j}^{*}=\frac{c_{1 j}\left(c_{2 j} h_{j}-e_{2 j}\right)-c_{2 j} e_{2 j}+\sqrt{\left(c_{1 j}\left(c_{2 j} h_{j}-e_{2 j}\right)-c_{2 j} e_{2 j}\right)^{2}-4 c_{1 j} c_{2 j} e_{1 j} e_{2 j}}}{2 c_{1 j} c_{2 j}}, p_{1 k}^{*}=0, p_{2 k}^{*}=0\right)
$$

Solving the dominant eigenvalue for $d$ leads to the invasion criterion (equation 9) in the main text.

Table A2. Equilibria when pollinator dispersal involves reproductive individuals

\begin{tabular}{lll}
\hline Isolated & Low dispersal & High dispersal \\
\hline $1.0,0,0,0$ & $0,0,0,0$ & $0,0,0,0$ \\
2. $0,0\left(p_{1 k}^{*}, p_{2 k}^{*}\right)_{I T}$ & $0,0\left(p_{1 k}^{*}, p_{2 k}^{*}\right)_{C T}$ & \\
3. $0,0\left(p_{1 k}^{*}, p_{2 k}^{*}\right)_{I C}$ & $0,0\left(p_{1 k}^{*}, p_{2 k}^{*}\right)_{C C}$ & \\
4. $\left(p_{1 j}^{*}, p_{2 j}^{*}\right)_{I T}, 0,0$ & $\left(p_{1 j}^{*}, p_{2 j}^{*}\right)_{C T}, 0,0$ & \\
5. $\left(p_{1 j}^{*}, p_{2 j}^{*}\right)_{I C}, 0,0$ & $\left(p_{1 j}^{*}, p_{2 j}^{*}\right)_{C C}, 0,0$ & \\
6. $\left(p_{1 j}^{*}, p_{2 j}^{*}\right)_{I T},\left(p_{1 j}^{*}, p_{2 j}^{*}\right)_{I C}$ & $\left(p_{1 j}^{*}, p_{2 j}^{*}\right)_{C C},\left(p_{1 j}^{*}, p_{2 j}^{*}\right)_{C C}$ & \\
7. $\left(p_{1 j}^{*}, p_{2 j}^{*}\right)_{I C},\left(p_{1 j}^{*}, p_{2 j}^{*}\right)_{I C}$ & $\left(p_{1 j}^{*}, p_{2 j}^{*}\right)_{C C},\left(p_{1 j}^{*}, p_{2 j}^{*}\right)_{C T}$ & $\left(p_{1 j}^{*}, p_{2 j}^{*}\right)_{C T},\left(p_{1 k}^{*}, p_{2 k}^{*}\right)_{C T}$ \\
8. $\left(p_{1 j}^{*}, p_{2 j}^{*}\right)_{I T},\left(p_{1 j}^{*}, p_{2 j}^{*}\right)_{I T}$ & $\left(p_{1 j}^{*}, p_{2 j}^{*}\right)_{C T},\left(p_{1 k}^{*}, p_{2 k}^{*}\right)_{C T}$ & $\left(p_{1 j}^{*}, p_{2 j}^{*}\right)_{C T},\left(p_{1 k}^{*}, p_{2 k}^{*}\right)_{C T}$ \\
9. $\left(p_{1 j}^{*}, p_{2 j}^{*}\right)_{I C},\left(p_{1 k}^{*}, p_{2 k}^{*}\right)_{I C}$ & $\left(p_{1 j}^{*}, p_{2 j}^{*}\right)_{C C},\left(p_{1 k}^{*}, p_{2 k}^{*}\right)_{C C}$ & \\
\hline
\end{tabular}

IT = isolated, threshold $; \mathrm{IC}=$ isolated, coexistence $\mathrm{CT}=$ connected, threshold $; \mathrm{CC}=$ connected, coexistence . 